\title{
The Contribution of African Capital Markets in the Diversification of European Investment Portfolios
}

\author{
Alexandrino Tavares Barreto ${ }^{1}$ \\ ${ }^{1}$ Faculty of Economics, University of Coimbra, Coimbra, Portugal \\ Correspondence: Alexandrino Tavares Barreto, Faculty of Economics, University of Coimbra, Coimbra, Portugal. \\ E-mail: alessandro021@live.com
}

Received: December 11, 2017

Accepted: January 10, 2018

Online Published: January 30, 2018

doi:10.5539/ijef.v10n3p1

URL: https://doi.org/10.5539/ijef.v10n3p1

\begin{abstract}
This study aims to evaluate the contribution of the African capital markets in the diversification of European investment portfolios. The study used the methodology based on the application of optimization models like Mean Variance (MV), Resample Michaud (RM), SemiVariance (SV), Mean Absolute Deviation (MAD) and Filtered Historical Simulation (FHS). In-Sample and Out-of-Sample approaches were used to analyze the data. The study results suggested the existence of a strong correlation between some African capital markets and European capital markets, that is, they tend to move in the same direction. The most important being the diversification of global portfolio with assets of African capital markets generate benefits for both types of investors; that is, it provides benefits in the return and reduce investment risk. Still, the study result suggested that the foreign investors should look for an African capital markets with a chance to maximize their wealth and diversify the investment risk in their portfolios. In the same order, the study result went further to elaborate contribute to on the advantage of the international diversification and furthermore contribute to the literature through application of the Filtered Historical Simulation (FHS) method in the optimization portfolio. This methodology, In addition to producing good results, is more restrained in the composition of investment portfolios than the other methods.
\end{abstract}

Keywords: African capital markets, diversification, European investment portfolios

\section{Introduction}

Globalization phenomenon has provided funds transfers between financial markets, with special attention given to the capital markets through the investor and fund managers that are seeking to invest in order to maximize wealth.

However, there are financiers that are willing to invest their assets in both domestic and international markets. This is done in order to minimize possible loss in the case of adverse events occurring in the domestic region that can negatively influence the expected result of their investments. The investors use diversification strategies to minimize risk and maximize return of portfolios in order to protect their investments.

Thus, this article attempts to tackle the issue of diversification in the international context, considering that European investors hold domestic portfolios where through diversification strategy, they include the African assets in their portfolios in order to reduce exposure to risk and to maximize return. This study aims to identify how the African capital markets will contribute to risk diversification of the European investment portfolios. Moreover, it compares capital markets to the level of dependency and exposure with respect to events that occur in these large markets.

Based on collected weekly data of the major capital markets in Europe-middle East and Africa markets and the methodology used, and in particular the application of the optimization models Mean Variance, Resample Michaud, SemiVariance, Mean Absolute Deviation and Filtered Historical Simulation, and using both In-Sample and Out-of-Sample approaches, our results suggest that African markets have a significant relationship with some of the European markets included in the study. The diversification of European portfolios with African assets generates benefits for the investor, i.e., provides benefits in return and reduces investment risk for both types of investors.

The study contribution to the literature is to test empirically the application of the Filtered Historical Simulation 
methodology in the portfolio optimization and contributes to the discussion about advantage of the context of international diversification. This FHS methodology, In addition of producing good results, reveals being more cautious in the constitution of investment portfolios than the other methods. However, this model presents lesser returns and higher risk than others models but their results follow the trend of the other models.

This work is structured in sections and subsections, where Section 2 presents literature review; Section 3 presents in detail the methodology used; Section 4 is reserved for the result analysis; and Section 5 is the bibliography references.

\section{Literature Review}

Several studies have shown that diversification in the international context bring advantages for investors holding composite portfolios with domestic and foreign securities.

According Mansourfar, Mohamad, and Hassan (2010) and Dimitriou and Kenourgios (2012) argue that diversification of the international portfolio has been a feature of the global capital market and potential benefits encourage the investors to diversify their investments. According to them, these benefits come from the fact that prices of international assets are less correlated and derive from different fundamental economic factors.

In addition, they point out the benefits of international diversification the investor's bets in the emerging markets and consequently have huge gains in the short term. According to Baele and Inghelbrecht (2009) and Chiou (2009), based on strong empirical support, potential gains from international diversification are still sufficient to justify a global asset allocation strategy rather than Industry/regional or local diversification.

Flavin and Panopoulou (2009) argue that diversification in the international context has long been advocated as an effective way to achieve a higher adjusted return on the investment risk in the domestic market, that is, facilitates risk sharing. According Rezayat and Yavas (2006) examined short-term co-movements between the five major stock markets (USA, UK, France, Germany and Japan) to assess the benefits of International Portfolio Diversification (IPD) and concluded that despite there is still room for diversification, but the benefits are minimal for American and European investors who would like to invest exclusively in these two major economic blocs (Europe and America). The study of MacDowell (2017) concluded IPD can provide gains and volatility reducing benefits. In the same way, recent study of Fletcher (2018) concluded that yet the benefits of international diversification are significant.

Laopodis (2005) argued that analysts said that financial integration among global capital markets has reduced IPD's benefits by increasing the correlation between equity markets. Coeurdacier and Guibaud (2011) argue that both theories and empirical evidence suggest that financial integration between countries has a positive impact on the correlation between equity markets, which tends to reduce IPD's benefits. The economic gains from international equity diversification are still substantial despite the growing markets correlation (Bouslama \& Ouda, 2014). On the same line, Maria and Eva (2012) concluded that as the markets become more integrated the co-movements between markets tend to rise, undermining the benefits of IPD. However, the study of Majdoub and Monsour (2013) on volatility spillovers between US and five (5) Islamic emergent equity markets found weak correlation over time. Bergin anda pyun (2016) argued that countries with returns more correlated reduce effective diversification and that international investors seek the diversification benefits through low correlated countries.

The major focus of studies on IPD focused on the portfolios of American, European and Asian investors focused on their diversification directed primarily at the assets of European and Asian capital markets, such as the studies (Note 1) of Odier and Solnik (1993), on a global investment where they found that it was advantageous for Japanese, British, German and American investors; Liljeblom et al. (1997) investigated the benefits of IPD from the point of view of Nordic investors; Ho et al. (1999), who reported that reducing the risk of loss through IPD would be of substantial benefit to Canadian investors; Rowland and Tesar (2004) and Gerke et al. (2005), who also examined the potential benefits of IPD from the perspective of the German investor; Dunis and Shannon (2005), who investigated stock markets in Southeast Asia (Malaysia, Philippines and Indonesia) and Central Asia (China, Belize, Taiwan, and India), where they found that IDP would be beneficial to investors in the USA; Kearney and Poti (2006), who used two conditional and unconditional estimation methods and analyzed the dynamics of correlation in five leading European capital markets, and Egret and Kocenda (2007), who analyzed the issue between Eastern European stock markets and Central Bank where they stated that there is no long-term bond between stock markets between these two blocs. Therefore, it can to said that about of the question of the International Portfolio Diversification in the African context, there are practically no studies done, except for the few references that, however, did not have a great impact on the African capital markets. 
The studies of Hassan et al. (2003) Bailey et al. (2005), Lagoarde-Segot and Lucey (2007), Yu e Hassan (2008) and Mansourfar, and Mohamad and Hassan (2010) on the stock markets in the countries of the Middle East and North Africa (MENA), concluded that there are many benefits of the portfolio diversification with titles of these regions are both in dollars and local currency. Furthermore, the study of Saiti, Bacha, and Masih (2014) concluded that Islamic countries (Note 2) provide better diversification benefits compared to the Far East countries with strong policies implications for the domestic and international investors in theirs portfolio diversification for hedging against unforeseen risks. In the same way, the study of Abbes and Trichilli (2015) suggest that Islamic stock (including Middle East and North Africa (Note 3)) could offer potential diversification benefits by considereing different economic grouping such as that in developed and emerging countries.

However, it was argued that these undervalued and under-investigated emerging markets could attract more value for portfolios in the future. To Mansourfar, Mohamad and Hassan (2010) in the last recent years, emerging equity markets have been subject of a large body of studies of international finance. Therefore, it makes sense to look at this issue as relevant in the context of the financial markets and the major economic blocks, particularly for Africa given the dynamism of their capital markets combined with economic growth in recent years and due to the financial crisis and lack of confidence that live in other great world capital markets. According Nie, Chong, Sambasivan, and Nassir (2014) the investors may not be able to obtain any benefits from diversifying their portfolio in developed stock markets in both ex-ante and ex-post periods. This is another reason that investors should look to Africa.

\section{Methodology}

\subsection{Data}

The sample consists of weekly data corresponding to the prices of the market index, collected in the Thomson Reuters Eikon. The database sample started on August 05, 2004 and ended on July 07, 2016, making a total of 624 weekly observations collected. Of the twenty four (24) major capital markets in Europe and Middle East, according to the classification given by MSCI World Index and thirteen (13) of the main African capital markets, as shows Tables 1 and 2. We consider weekly returns measured in USA Dollars. To measure the return, risk level and composition of investment portfolios, we propose the following optimization models like Mean Variance (MV), Resample Michaud (RM), SemiVariance (SV), Mean Absolute Deviation (MAD) and Filtered Historical Simulation (FHS). To evaluate the relationship between capital markets, we use the correlation coefficient. We resort to Matlab software for the application of the optimization models and Excel to make the graphics of efficient portfolios and estimate performance indicators.

Table 1. African capital markets

\begin{tabular}{llll}
\hline Continent Africa & Country & Currency/Code & Market Index (Name) \\
\hline South Africa & Rand (R) & FTSE/JSE Africa top 40 index \\
Egypt & Egyptian Pound (EGP) & Egyptian EGX30 index \\
Morocco & Moroccan Dirham (MAD) & Moroccan All Share MASI \\
Tunisia & Tunisian Dinar (TND) & Tunindex \\
Botswana & Botswana Pula (BWP) & BSE Domestic Company DCIBT \\
Malawi & Malawian Kwacha (MWK) & Malawi All share Index (MASI) \\
Mauritius & Mauritian Rupi (MUR) & Semdex MDEX \\
Namibia & Namibian Dollar (NAD) & Namibia Stock Exchange (NSX) \\
Nigeria & Nigerian Naira (NGN) & NSE Index 30 (NSEINDX:IND) \\
Kenya & Kenyan Shilling (KES) & Kenya NSE 20 (NSE20) \\
Uganda & Ugandan Shilling (UGX) & Uganda All Share (ALSIUG) \\
Zambia & Zambian Kwacha (ZMK) & LSE All Share (LASILZ) \\
Rep Democratic of Congo & Congolese Franc (CDF) & All Share Index \\
Costa do Marfim/Cote D'ivoire & XOF & All Share Index \\
\hline
\end{tabular}

The table 1 shows all African capital market include in this study. Therefore. the capital markets did not meet the requirements of the sample between periods start from August 05, 2004 to July 07, 2016 they were excluded of the study. In the first column shows the countries. second the local currency index quotation and the third column we find the main market index for each country. 
Table 2. European and Middle East capital markets

\begin{tabular}{|c|c|c|c|}
\hline Continent Europe and Middle East & Country & Currency/Code & Market Index (Name) \\
\hline & Germany & Euro(€) & DAX index \\
\hline & United Kingdom & Euro(€) & FTSE 100 INDEX (FTSE) \\
\hline & France & $\operatorname{Euro}(€)$ & CAC 40 index \\
\hline & Italy & Euro( $€)$ & FTSE MIB index \\
\hline & Spain & Euro(€) & IBEX 35 index \\
\hline & Austria & $\operatorname{Euro}(€)$ & ATX (ATX) \\
\hline & Switzerland & Swiss Franc (CHF) & SMI (SSMI \\
\hline & Belgium & Euro(€) & BEL20 (BFX) \\
\hline & Denmark & Danish Krone (DKK) & OMX COPENHAGEN 20 (OMXC20) \\
\hline & Finland & Euro(€) & OMX Helsinki 25 (OMXH25) \\
\hline & Ireland & Euro(€) & ISEQ Overall (ISEQ) \\
\hline & Israel & Israeli Shekel (ILS) & Tel Aviv 25 Index (TA25) \\
\hline & Netherlands & Euro(€) & $\operatorname{AEX}(\mathrm{AEX})$ \\
\hline & Norway & Norwegian Krone (NOK) & Oslo Stock Exchange All Share Index \\
\hline & Portugal & Euro(€) & PSI 20 (PSI20) \\
\hline & Sweden & Swedish Krona (SEK) & OMX Stockholm 30 (0MXS30) \\
\hline & Czech Republic & Czech Koruna (CZK) & $\mathrm{PX}(\mathrm{PX})$ \\
\hline & Greece & Euro(€) & Athens General (ATG) \\
\hline & Hungary & Hungarian Forint (HUF ) & Budapest SE (BUX) \\
\hline & Poland & Polish Zloty (PLN) & WIG 20 (WIG20) \\
\hline & Qatar & Qatari Riyal (QAR) & Stock Market DOHA (QSI) \\
\hline & Russia & Russian Ruble (RUB) & $\operatorname{MICEX}(\mathrm{MCX})$ \\
\hline & Turkey & Turkish Lira (TRY) & BIST 100 (XU100) \\
\hline & United Arab Emirates & AED & ADX General (ADI) \\
\hline
\end{tabular}

The table 2 shows all the mains European and Middle East capital markets included in study according to the MSCI Word Index classify in the develop and emergent markets. Therefore. the capital markets did not meet the requirements of the sample between periods start from August 05, 2004 to July 07, 2016 they were excluded of the study. In the first column shows the countries. second the local currency index quotation and the third column we find the main market index for each country.

\subsection{In-Sample and Out-of-Sample Approaches}

In the first stage, the In-Sample approach for the entire period $\mathrm{T}$ of returns observations, where the different investment distribution strategies are plotted and represented by curves of efficient frontiers. Then, to evaluate and measure portfolio performances and the contribution to diversification we propose Sharpe Ratio and Sortino Ratio such as Lagoarde-Segot e Lucey (2007) and to measure the contribution of portfolio diversification, we proposed the measures suggested by Liang and McIntosh (1999).

In the second phase, in line with the works of De Miguel et al. (2009), Daskalaki and Skiadopoulos (2011), Bessler, Opfer, and Wolff (2014), we applied the Rolling Sample approach, in order to understand the contribution of African assets in the diversification of European investment portfolios. Rolling Sample methodology consists in considering a window with $\mathrm{M}$ observations for a given sub-period. The next step is to rolling this window adding 1 more observation in the window $\mathrm{M}$ (we considered $\mathrm{M}=5$ years, corresponding to the 260 observations) and drop the first observation of the window $\mathrm{M}$, and calculate tangential portfolios, that maximizes performance. This process is repeated always adding one more new observation in the window and dropping the oldest observation and so on, determining the optimum portfolios for each window bearing until it reaches the total observation (the total of 363 portfolios weights for Out-of-Sample analyses). The Out-of-Sample evaluation is based on the performance of the following statistics like Excess return (ER), Risk (R), Sharpe Ratio (SR) and Sortino Ratio (S) to realize the contribution of the assets of African markets in the diversification of European investment portfolios. However, before following this Rolling Sample methodology, we need to divide the sample into two sub-periods of 5 years ( first sub-period started from August 05, 2004 (Note 4) to July 03, 2009 and second sub-period started from July 03, 2009 to July 07, 2016). Therefore, for the Out-of-Sample analysis, we have 2 sub-periods to evaluate the performance of the investment distribution strategies. 
To evaluate the contribution of the African assets in the diversification of European investment portfolios, we defined some possible strategies that investors can to follow. However, it is important to note that nothing assures us that European investors can adopt these strategies because as you know, each investor has his own profile when it comes to investment. We assume that a rational investor can choose these two strategies here presented:

Strategy 1: The investor makes an optimal distribution of $100 \%$ of his investment in Europe's markets. We consider this portfolio composition such as domestic portfolios.

Strategy 2: The investor chooses to make an optimal distribution of $100 \%$ of his investment between European and African capital markets. Therefore, this is diversification strategy.

The In-Sample analysis for each strategy are made of 50 optimal portfolios (Note 5) that comprise the efficient frontiers based on risk and return. To evaluate the performance of the strategies and test the statistical significance, we considered two (2) null hypotheses:

$$
\begin{gathered}
H_{0}: S R 2>S R 1 \\
H_{0}: S 2>S 1
\end{gathered}
$$

SR2 and S2 are the values of the Sharpe Ratio and Sortino Ratio index performances for strategy 2; SR1 and S1 are the values of the Sharpe Ratio and Sortino Ratio index performances for Strategy 1. The objective is to evaluate whether the differences between the performances of the strategies are statistically significant, considering a $1 \%$ significance level for both analyses. Therefore, we compare the diversification strategy with an undiversified strategy that is Strategy 1.

\subsection{Portfolio Optimization Models}

\subsubsection{Mean Variance (MV)}

The first work about portfolio optimization was developed by Markowitz (1952), known as Mean Variance model (MV). This model suggests that making decisions about portfolio composition risk and return must be decision criteria. The risk measure is standard deviation and the return measure is given by the average value of assets returns. Although it is a model criticized, it is a model widely used in financial studies. The Markowitz paradigm expects return and volatility to be relevant aspects that investors take in consideration when making decisions about portfolio composition. Thus, for the risk adverse investors the expectation to minimize risk to a given return limit according Markowitz, can be expressed by:

$$
\text { Minimize Portfolio risk }=\sqrt{\sum_{\mathrm{i}=1}^{\mathrm{N}} \sum_{\mathrm{j}=1, \mathrm{j} \neq \mathrm{i}}^{\mathrm{N}}\left(\mathrm{x}_{\mathrm{i}} \mathrm{x}_{\mathrm{j}} \rho_{\mathrm{ij}} \sigma_{\mathrm{i}} \sigma_{\mathrm{j}}\right)}
$$

subject to a minimum expected return given by:

$$
\sum_{i=1}^{N} x_{i} \bar{r}_{l} \geq r_{C}
$$

total investment in the portfolio given by:

$$
\sum_{i=1}^{N} x_{i}=1
$$

and to ensure that there is no negative investment given by:

$$
x_{i} \geq 0 \forall i
$$

$\mathrm{N}$ is the number of assets; $\mathrm{x}_{\mathrm{i}}$ and $\mathrm{x}_{\mathrm{j}}$ are the weights of the assets in the portfolio; $\sigma_{\mathrm{i}}$ and $\sigma_{\mathrm{j}}$ are the standard deviations of the assets $\mathrm{i}$ and $\mathrm{j} ; \rho_{i j}$ is the correlation between assets $\mathrm{i}$ and $\mathrm{j} ; \bar{r}_{l}$ corresponds to the average return of the asset and $\mathrm{r}_{c}$ corresponds to the minimum desired portfolio return.

\subsubsection{Resample Michaud (RM)}

This method was developed by Michaud (1998) and according to Becker, Gürtler and Hibbeln (2015) the basic concept of Michaud comprises of three aspects: (1) a generation of a sequence of returns, which are statistically equivalent to the actual times series of returns, through a Monte Carlo Simulation; (2) the subsequent determination of portfolio weights for every resample and (3) the averaging over the obtained portfolio weights to obtain the optimal portfolio weights according Michaud. This method can be considered a "sophistication" of the MV model but based on the simulation method.

The algorithm that explains how to implement this method is described as follows: (1) From the original database, two parameters are estimated, the vector of expected excess returns $(\mu)$ and the variance-covariance matrix $\left(\sum\right)$; (2) Resample applying multivariate normal distribution with mean $\mu$ and covariance $\sum$ considering T draws. For each resample that is generated there is a new mean $\mu$ and covariance $\sum$ to estimate optimal portfolio 
weights over T draws; and (3) Choosing the optimal portfolio weights depends on the required portfolio number. Then, are estimated the portfolio risk and portfolio return that make up the Efficient Frontiers by Michaud.

\subsubsection{SemiVariance (SV)}

This model has emerged as an alternative to the Mean-Variance model (MV), in order to remedy its shortcomings raised by scholars and researchers in the field of finance. Thus, Markowitz himself (1959) recognized the shortcomings of the MV model and proposed the SV model as the most appropriate measure of risk for investment portfolios. Thus, in general, according to Markowitz (1959), cited by Bond and Satchell (2002), and Estrada (2008), the SV model for an individual asset is defined as follows:

$$
S V=\frac{\sum_{j=1}^{T}\left\{\min \left[0,\left(r_{i}-\bar{r}_{i}\right)\right]\right\}^{2}}{T}
$$

The standard deviation of the Semi-Variance of an asset is given by:

$$
\mathrm{SV}=\sqrt{\frac{\sum_{\mathrm{j}=1}^{\mathrm{T}}\left\{\min \left[0,\left(\mathrm{r}_{\mathrm{it}}-\overline{\mathrm{r}}_{i}\right)\right]\right\}^{2}}{\mathrm{~T}}}
$$

The Semi-Variance of an investment portfolio $\left(\mathrm{SV}_{\mathrm{C}}\right)$ is given as:

$$
\mathrm{SV}_{C}=\frac{\sum_{\mathrm{j}=1}^{\mathrm{T}}\left\{\min \left[0,\left(\mathrm{r}_{C t}-\overline{\mathrm{r}}_{C}\right)\right]\right\}^{2}}{\mathrm{~T}}
$$

However, there are authors (e.g., Estrada, 2008) that suggest the estimation portfolio Semi-Variance approach by the expression:

$$
\mathrm{SV}_{C} \approx \sum_{\mathrm{i}=1}^{\mathrm{N}} \sum_{\mathrm{j}=1}^{\mathrm{N}}\left(\mathrm{x}_{\mathrm{i}} \mathrm{x}_{j} S C_{i j}\right)
$$

According to Estrada (2008) and Cumova and Nawrocki (2011) Semi-Covariance (SC) between the assets of the portfolios is estimated as:

$$
S C_{i j}=\frac{1}{T} \sum_{t=1}^{T}\left[\operatorname{Min}\left(r_{i t}-\bar{r}_{i}, 0\right) \cdot \operatorname{Min}\left(r_{j t}-\bar{r}_{j}, 0\right)\right]
$$

The expected return of an investment portfolio is obtained from the following expression:

$$
E\left(R_{C}\right)=\sum_{i=1}^{N} x_{i} \bar{r}_{i}
$$

The mathematical formulation of the portfolio optimization problem using this model has as objective function to minimize the SV subject to certain restrictions as expressions as follows:

Minimize

$$
\mathrm{SV}_{C} \approx \sqrt{\sum_{\mathrm{i}=1}^{\mathrm{N}} \sum_{\mathrm{j}=1}^{\mathrm{N}}\left(\mathrm{x}_{\mathrm{i}} \mathrm{x}_{j} S C_{i j}\right)}
$$

subject to a minimum expected return is given by:

total investment in the portfolio is given by:

$$
\sum_{i=1}^{N} x_{i} \bar{r}_{l} \geq r_{C}
$$

$$
\sum_{i=1}^{N} x_{i}=1
$$

and to ensure there is no negative investment is given by:

$$
x_{i} \geq 0 \forall i
$$

where, $\mathrm{T}$ is the size of the observation period; $\mathrm{t}$ is the sample period over $\mathrm{T} ; \mathrm{r}_{i t}, \mathrm{r}_{\mathrm{jt}}$ e $\mathrm{r}_{C t}$ are the observed returns of assets $i, j$ and portfolio $c$ in the period $t ; \bar{r}_{i}, \bar{r}_{j}$ e $\bar{r}_{C}$ are the observed mean returns of the assets and portfolio. In the maximization problem, the objective function is portfolio returns subjected to restrictions.

\subsubsection{Mean Absolute Deviation (MAD)}

To overcome the shortcomings of the model Mean Variance, Konno and Yamasaki (1991) suggested the model Mean Absolute Deviation (MAD) as linear programming or linear optimization of portfolios, where the risk measure is the designed Average Deviation Absolute. Still, According these authors, the MAD is based on dividing the distribution of a variable randomized into two groups, those above and below the average and estimates the absolute deviations of observations in each group from the average. Thus, these are properties make MAD preferred over the standard deviation, and especially when the distribution is not normal. It can still be designated as a model used as a measure of risk in the portfolio optimization (Miller \& Ruszczynski, 2008), taking into consideration that the relevance for investors is to minimize the risks and maximize returns for their 
portfolios. It is a very general measure of risk and can be used in other risk management practices (Xue \& Titterington, 2011). The linear formulation takes advantage of a less computational effort (unlike quadratic formulation) and more applicability in practical terms (Moon \& Yao, 2011). The authors formulated as follows:

$$
M A D_{C}=\frac{1}{T} \sum_{t=1}^{T}\left|\sum_{j=1}^{n}\left(r_{j t}-\bar{r}_{j}\right) x_{j}\right|
$$

The mathematical formulation of the portfolio optimization problem posed by this model suggested by Konno and Yamazaki (1991) can be summarized by the following expressions:

$$
\text { Minimize } M A D_{C}=\frac{1}{\mathrm{~T}} \sum_{\mathrm{t}=1}^{\mathrm{T}}\left|\sum_{\mathrm{j}=1}^{\mathrm{n}}\left(\mathrm{r}_{\mathrm{jt}}-\mathrm{r}_{\mathrm{j}}\right) \mathrm{x}_{\mathrm{j}}\right|
$$

subject to a minimum expected return is given by:

$$
\sum_{i=1}^{N} x_{i} \bar{r}_{l} \geq r_{C}
$$

total investment in the portfolio given by:

$$
\sum_{i=1}^{N} x_{i}=1
$$

and to ensure there are no negative investments is given by:

$$
x_{i} \geq 0 \forall i \text {. }
$$

\subsubsection{Filtered Historical Simulation (FHS)}

This method is quite credible and acceptable among scholars and researchers. Some articles have addressed this method and it is use in the estimation of portfolio risk, but yet, unknown articles have used the FHS in portfolio optimization, and this is one of the important contributions of this work. The FHS is one of the methods of Value-at-Risk (VaR) that combines the traditional method Historical Simulation (HS) with volatility models (Garch or Egarch).

Thus, through a simple, clear language all steps for implementing the FHS method can to be shown according Giannopoulos and Tunaru (2005). The algorithm to implementation in determining the level of risk and portfolio optimization, requires five (5) steps described below:

(1) Application of the Historical Simulation method;

(2) Estimation of volatilities of returns series of the portfolios through the Garch $(1,1)$ model;

(3) Estimation of Residual returns standardized, obtained by dividing the residual value of returns by the respective variance;

(4) Application Bootstrapping method where in for each standardized return period t randomly generated multiplies the variance of the period $t+1$; and finally; and

(5) estimates the VaR through the percentile of returns, considering a certain confidence interval and a significance level, and period of portfolio tenure.

\subsubsection{Historical Simulation Method (HS)}

The application of VaR method is quite simple and requires some steps: (1) The estimation of periodic returns of the assets that make up the initial portfolio; (2) Periodic portfolios, adding the products of periodicals returns of each asset at its initial weight is estimated to (1/N, where $\mathrm{N}$ is the total number of assets) and (3) Considering a certain significance level and period detention portfolios, estimated VaR, which is given by the expression:

$$
\text { VaR_HS }=- \text { Percentil }\left\{\left\{\sum_{\mathrm{i}=1}^{\mathrm{N}} \mathrm{x}_{\mathrm{i}} \mathrm{r}_{i}\right\}^{m}, \alpha \%\right\}
$$

Where, $r_{i}$ is the periodic return of the asset $i$ and $m$ refers to the observation period ( $m$ only illustrates the period that corresponds to summation. It does not have any mathematical effect on the formula) and $\alpha \%$ corresponds to the specified significance level.

\subsubsection{Garch Volatility Model}

It is assumed the Garch $(1,1)$ model is to estimate periodic variances of portfolios. However, nothing ensures the possibility of the historical returns of the assets assuming a normal distribution or t-student. Considering the simple Garch model, standardized residual returns are estimated by the expression:

$$
z_{t+1}=\frac{R_{t+1}}{\sigma_{t+1}}
$$

Where the variance is given as: 


$$
\sigma_{t+1}^{2}=\omega+\varphi R_{t}^{2}+\beta \sigma_{t}^{2}
$$

and, $\omega, \varphi$ and $\beta$ are model parameters whose estimation can be by maximizing the sum of the function Maximum Likelihood Estimation (MLE) which is given by the expression, similar Aldrich (1997):

$$
M L E_{t+1}=L N\left(\frac{1}{\sqrt{\left.2 \pi \sigma_{t+1}^{2}\right)}} * \exp \left(-0,5 * \frac{R_{t+1}^{2}}{\sigma_{t+1}^{2}}\right)\right)
$$

Where $R_{t+1}$ is the residual value of the return; $R_{t}{ }^{2}$ is the residual value squared and $\sigma_{t}^{2}$ is the unconditional variance in period $\mathrm{t}$.

\subsubsection{Bootstrapping Method}

This method, given a certain period of detention portfolios, from observations of standardized residual returns, randomly generates return for period $t$ to be multiplied by the variance in period $t+1$. Made the random returns of portfolios, will be estimated the FHS VaR, which can be given by the expression:

$$
\text { VaR_FHS } \left.=- \text { Percentil }\{\text { random returns }\}^{m}, \alpha \%\right\}
$$

The use of this method in portfolio optimization requires some care because the process is a little different from other methods, although apparently it has an almost similar mathematical formulation. There are two (2) objectives function to consider:

$$
\begin{gathered}
\text { MinimizarVaR_HS }==-\operatorname{Percentil}\left\{\left\{\sum_{\mathrm{i}=1}^{\mathrm{N}} \mathrm{x}_{\mathrm{i}} \mathrm{r}_{i}\right\}^{m}, \alpha \%\right\} \\
\text { VaR_FHS }=- \text { Percentil }\left\{\{\text { random returns }\}^{m}, \alpha \%\right\}
\end{gathered}
$$

subject to a minimum expected return is given by:

$$
\sum_{i=1}^{N} x_{i} \bar{r}_{l} \geq r_{C}
$$

total investment in the portfolio is given by:

$$
\sum_{i=1}^{N} x_{i}=1
$$

and to ensure there is no negative investment is given by:

$$
x_{i} \geq 0 \forall i \text {. }
$$

\subsection{Performance Measures and Contribution of Portfolio Diversification Strategies}

\subsubsection{Sharpe Ratio (SR)}

The SR index of a particular investment strategy is measured by the ratio between the risk premiums or excess return and risk of strategy $i$ as the expression (Sharpe, 1994):

$$
I S_{i}=\frac{\widehat{\mu}_{i}}{\sigma_{i}}
$$

where, $\mu_{i}$ corresponds to the risk premium (corresponding to the difference between portfolio return and risk-free rate asset (Note 6)) and $\sigma_{i}$ is the risk of strategy i. This indicator shows how much the investor receives the strategy i defined for each unit of risk associated with the strategy $i$. The higher value for this measure, indicate higher quality of the investment in the strategy i. Assuming a normal distribution, to determine whether SR and S between the strategies are statistically significant, we propose Two-sample t-test, according with the Matlab code in the appendices to test the null hypotheses.

\subsubsection{Sortino Ratio (S)}

Such as the Sharpe Ratio, the Sortino Ratio is also an important statistical indicator used to measure the investment portfolio performance. Dr. Frank Sortino proposed it in the 80s. However, it differs from the Sharpe Ratio because it uses the standard deviation of negative returns while the Sharpe Ratio uses the standard deviation of positive and negative returns. This is one of the reasons appointed as insufficient of MV model. The Sortino Ratio is a modification of the Sharpe Ratio, and can be expressed by:

$$
S=\frac{\widehat{\mu}_{i}}{\text { Downside risk (Note 7) }}
$$

\subsubsection{Contribution Measures of Portfolio Diversification}

To measure the contribution of assets of African capital markets in Europe's portfolio, we propose three measures 
according to Liang and McIntosh (1999):

Overall Benefit $\left(\mathrm{OB}_{\mathrm{i}}\right)$ : This indicator measures the general benefit of the investment diversification effect, that is, it measures benefits in reducing risk and return. It is given by the following expression:

$$
O B_{i}=\left(R_{i}-R_{f}\right)-\left(\rho \sigma_{i} / \sigma_{m}\right)\left(R_{m}-R_{f}\right)
$$

Diversification Benefit $\left(\mathrm{DB}_{\mathrm{i}}\right)$ : This indicator measures only the benefits of Investment diversification in the risk reduction. It is given as follows:

$$
D B_{i}=\left(R_{m}-R_{f}\right)\left(1-\rho \sigma_{i} / \sigma_{m}\right)
$$

Return Benefit $\left(\mathrm{RB}_{\mathrm{i}}\right)$ : This indicator measures only the benefits of investment diversification in the return. It is represented by the expression:

$$
R B_{i}=R_{i}-R_{m}
$$

Where, $R_{m}=$ existing portfolio return $\mathrm{m} ; \sigma_{m}=$ volatility of portfolio $\mathrm{m} ; R_{i}=$ Return i proposed investment; $\sigma_{i}=$ Volatility i proposed investment; $\rho=$ correlation coefficient between portfolio $\mathrm{m}$ and investment $\mathrm{i}$; and $R_{f}=$ risk-free rate.

\section{Discussion and Results}

\subsection{In- Sample Analysis}

In analyzing Table 3, strong positive correlation between African capital markets and European capital markets can be found. However, some African capital markets, such as Nigerian, Democratic Republic Gongo and Cote D'ivoire show weak correlation with European capital markets.

The study results show that African capital markets have tendencies to follow the behavior of European capital markets. The Important African capital markets, such as South African, Namibia, Egypt, Morocco, Tunisia and Kenya presents significant and positive correlation with European capital markets between periods of data analysis start from $5^{\text {th }}$ August, 2004 to $7^{\text {th }}$ July, 2016 as seen in the test p-value results correlation shown in table 4 considering the significance level of $1 \%$.

For In-Sample analysis, Tables 5 to 9 shows the results for each strategy based on trade-off risk and return, where we can to find European portfolio optimization before and after diversification with their respective performances based on optimization models. The results show that the diversification of European investment portfolio with African assets, contributes to reduce the risk and maximize the return.

As Figures 1 and 2 show, we can to see different efficient frontiers for each of the optimization models used in this study that represents the two (2) investment strategies. To all optimization models, the strategy of the diversification of European portfolio with assets of the African capital market show higher return than European investment portfolio without diversification, such as illustrated by Table 10.

On the other hand, on the same table, for MV and SV models the diversification of European investment portfolio with assets of African capital market, increase the risk level but for RM, MAD and FHS models, it does not increase. In other words, for these models, diversification of European portfolio with assets of African capital markets reduces the risk level. However, the diversification strategy of European investment portfolio with assets of African capital markets presents better performance than European investment portfolio without diversification, according to the results of the Sharpe Ratio and Sortino Ratio performance in the figures 3 and 4, where it's observed the African capital markets in the diversification of European investment portfolio. These results are statistically significant for all models included in the study, since it not reject the null hypotheses, according the results of Table 11 .

Therefore, overall the investment strategy 2 shows better performance than strategy 1 . The real contribution of the diversification of European investment portfolio with assets of African capital markets is illustrated in Table 12. For all models, this strategy generates benefit in diversification and return benefit, such as shown in the table.

We can to see weak contribution of the European capital markets and Middle East in the diversification of European investment portfolio, being outweighed by large contributions of the African capital markets. According Tables 13 to 18 , the African capital markets with great performance in the composition of the portfolio diversifying Europe with higher weights are: Cote D'ivoire, Republic Democratic Gongo, Zambia and Tunisia. Already with less weight, we find the following markets: Botswana, Mauritius, Egypt and Uganda. The study data analyses show that in general the African capital markets are more efficient than European capital markets.

In summary, the study In-Sample analysis of the database in the period considered, allows us to see that the diversification of European investment portfolio with assets of African markets contributes to reduce risk and 
maximize return of the portfolio, where investor that prefer high level of risk at the expense of a high return. Yet, how our results shows, Tables 5 to 9, for all models, even for investors that prefer Minimum Portfolio Variance (MPV), the diversification of European investment portfolio with African assets, reduce risk and maximize return. Even if the diversification of European investment portfolio with African assets increased risk, the benefits in return compensate the increase risk.

\subsection{Out-of-Sample Analysis}

In this analysis, we also analyzed the contribution of the African capital market in the diversification of European investment portfolio using Out-of-Sample analysis. Furthermore, the objective of this methodology is to analyze the portfolio performances measured by Excess Return (ER), Risk (R), Sharpe Ratio (SR) and Sortino Ratio $(S)$, over the period where it is applied by the Rolling Sample approach.

Out-of-Sample results show that the strategy of diversification of European investment portfolio with assets of African market present better performance measured by Excess Return, Risk, Sharpe Ratio and Sortino Ratio than European investment portfolio according to the models as shown in Table 19 and figure 5 to 8 . To test statistically the investment performances measured by Sharpe Ratio and Sortino Ratio where the table 20 provides the test results. For all optimization models MV, RM, SV, MAD and FHS, show not rejection of the null hypotheses. The result shows high positive value of $t$ - statistic and p-value lesser $1 \%$. It means that the superiority of the investment performances of the strategy of the diversification of European investment portfolio with assets of African capital markets is statically significant.

Table 3. Correlation coefficients between capital markets

\begin{tabular}{|c|c|c|c|c|c|c|c|c|c|c|c|c|c|}
\hline & Namibia & Nigerian & Gongo & $\begin{array}{c}\text { Cote } \\
\text { D'Ivoire }\end{array}$ & Egypt & Morrocco & o Tunisia & Botswana & a Mauritius & Kenya & Uganda & aZambia & $\begin{array}{l}\text { South } \\
\text { Africa }\end{array}$ \\
\hline Germany & 0.40 & 0.05 & 0.04 & 0.11 & 0.14 & 0.24 & 0.15 & 0.22 & 0.21 & 0.19 & 0.11 & 0.08 & 0.66 \\
\hline$U K$ & 0.47 & 0.13 & 0.04 & 0.20 & 0.14 & 0.22 & 0.11 & 0.25 & 0.22 & 0.22 & 0.12 & 0.10 & 0.75 \\
\hline France & 0.40 & 0.07 & 0.04 & 0.14 & 0.12 & 0.24 & 0.13 & 0.23 & 0.17 & 0.16 & 0.06 & 0.05 & 0.67 \\
\hline Italy & 0.27 & 0.07 & 0.04 & 0.10 & 0.19 & 0.28 & 0.15 & 0.22 & 0.16 & 0.20 & 0.08 & 0.07 & 0.53 \\
\hline Spain & 0.31 & 0.06 & -0.01 & 0.11 & 0.10 & 0.23 & 0.16 & 0.24 & 0.11 & 0.16 & 0.07 & 0.04 & 0.55 \\
\hline Austrian & 0.36 & 0.11 & 0.03 & 0.07 & 0.27 & 0.31 & 0.18 & 0.26 & 0.25 & 0.22 & 0.09 & 0.13 & 0.60 \\
\hline SWISS & 0.33 & 0.05 & 0.04 & 0.01 & 0.19 & 0.24 & 0.11 & 0.19 & 0.20 & 0.15 & 0.08 & 0.04 & 0.56 \\
\hline Belgium & 0.39 & 0.08 & 0.02 & 0.24 & 0.16 & 0.27 & 0.14 & 0.24 & 0.22 & 0.20 & 0.09 & 0.09 & 0.59 \\
\hline Denmark & 0.31 & 0.11 & 0.03 & 0.10 & 0.15 & 0.28 & 0.11 & 0.20 & 0.18 & 0.18 & 0.08 & 0.08 & 0.59 \\
\hline Finland & 0.36 & 0.05 & 0.03 & 0.07 & 0.11 & 0.25 & 0.10 & 0.19 & 0.19 & 0.14 & 0.02 & 0.04 & 0.66 \\
\hline Ireland & 0.27 & 0.10 & 0.03 & 0.16 & 0.19 & 0.23 & 0.11 & 0.24 & 0.28 & 0.18 & 0.08 & 0.14 & 0.50 \\
\hline Israel & 0.25 & 0.08 & 0.05 & 0.16 & 0.19 & 0.21 & 0.04 & 0.14 & 0.20 & 0.19 & 0.08 & 0.14 & 0.38 \\
\hline Netherlands & 0.41 & 0.09 & 0.04 & 0.22 & 0.15 & 0.30 & 0.10 & 0.23 & 0.23 & 0.18 & 0.06 & 0.11 & 0.70 \\
\hline Norway & 0.45 & 0.06 & 0.05 & 0.26 & 0.12 & 0.26 & 0.09 & 0.21 & 0.20 & 0.15 & 0.05 & 0.12 & 0.73 \\
\hline Portugal & 0.22 & 0.02 & 0.06 & 0.02 & 0.12 & 0.23 & 0.16 & 0.17 & 0.12 & 0.13 & 0.05 & 0.04 & 0.44 \\
\hline Sweden & 0.43 & 0.08 & 0.04 & 0.13 & 0.10 & 0.28 & 0.08 & 0.25 & 0.21 & 0.13 & 0.04 & 0.05 & 0.67 \\
\hline Zech Republic & 0.38 & 0.10 & 0.04 & 0.17 & 0.28 & 0.26 & 0.15 & 0.25 & 0.28 & 0.23 & 0.11 & 0.11 & 0.54 \\
\hline Greece & 0.39 & 0.02 & 0.00 & 0.28 & 0.00 & 0.10 & 0.06 & 0.10 & 0.01 & 0.07 & 0.02 & 0.00 & 0.28 \\
\hline Hungary & 0.35 & 0.09 & -0.02 & 0.18 & 0.27 & 0.24 & 0.16 & 0.36 & 0.28 & 0.26 & 0.18 & 0.15 & 0.53 \\
\hline Poland & 0.41 & 0.08 & 0.02 & 0.21 & 0.22 & 0.21 & 0.10 & 0.34 & 0.27 & 0.21 & 0.12 & 0.15 & 0.62 \\
\hline Qatar & 0.14 & 0.13 & -0.04 & 0.03 & 0.27 & 0.14 & 0.13 & 0.08 & 0.21 & 0.20 & 0.14 & 0.12 & 0.19 \\
\hline Russian & 0.39 & 0.11 & -0.02 & 0.11 & 0.21 & 0.20 & 0.10 & 0.22 & 0.27 & 0.21 & 0.09 & 0.12 & 0.54 \\
\hline Turkey & 0.34 & 0.01 & 0.00 & 0.18 & 0.20 & 0.11 & 0.10 & 0.28 & 0.25 & 0.20 & 0.14 & 0.13 & 0.51 \\
\hline$U A E$ & 0.23 & 0.11 & 0.07 & 0.05 & 0.14 & 0.22 & 0.13 & 0.08 & 0.18 & 0.10 & 0.07 & 0.03 & 0.22 \\
\hline
\end{tabular}

The Table 3 shows the correlation level between returns of African and European capital markets considering 1\% like a significance level. We recall that the returns was measured in dollar USA. we can find strong positive correlation between African capital market and European capital markets. However. some African capital markets. such as Nigerian. Democratic Republic Gongo and Cote D'ivoire show weak correlation with European capital markets. Our results show that African capital markets have tendencies to follow the behavior of European capital markets. in the same direction and opposite. as we can to see negative values of correlation. The main African capital markets. such as South African. Namibia. Egypt. Morocco. Tunisia and Kenya presents high and positive correlation with European capital markets between periods of data analysis. 
The study Out-of-Sample results show that the strategy of diversification of European investment portfolio with assets of African capital market present better performances measured by Sortino Ratio than European investment portfolio for all optimization models included in this study as we can to see in Table 20 where for all optimization models no reject the null hypotheses. It means that there are superiority of the Sharpe and Sortino Ratio Performances of the strategy of diversification of European investment portfolio with assets of African capital markets is statically significant for $1 \%$ of the significance level. Finally, such In-sample analysis shows us, the Out-of-Sample analysis also show us a great contribution of the African capital market in the European investment portfolio composition as we can to see in table 21 .

\subsection{Summary}

The study data analysis from the period of $5^{\text {th }}$ August, 2004 to $7^{\text {th }}$ July, 2016 using the optimization models Mean Variance (MV), Resample Michaud (RM), SemiVariance (SV), Mean Absolute Deviation (MAD) and Filtered Historical Simulation (FHS) allows us the conclusion that the diversification of European investment portfolio with assets of the African capital markets contributes to minimize risk and maximize the return of the portfolio for the risk averse investors. On the other hand, for risk loving investors, the diversification of European investment portfolio with assets of African capital markets increase the level of risk, but the benefit returns compensate for the risk increase. Our results are in line with other works, such as Lagoarde-Segot e Lucey (2007), Yu and Hassan (2008) and Mansourfar, Mohamad and Hassan (2010), Bacha and Masih (2014), Trichilli (2015), MacDowell (2017), Fletcher (2018) and others in the context of the International Diversification.

Table 4. P-value test of the correlation coefficients between capital markets

\begin{tabular}{|c|c|c|c|c|c|c|c|c|c|c|c|c|c|}
\hline & Namibia & Nigerian & Gongo & Cote D'Ivoire & Egypt & Morroc & Tunisia & Botswana & Mauritius & Kenya & Uganda & Zambia & South Africa \\
\hline Germany & 0.000 & 0.219 & 0.265 & 0.004 & 0.001 & 0.000 & 0.000 & 0.000 & 0.000 & 0.000 & 0.009 & 0.902 & 0.000 \\
\hline$U K$ & 0.000 & 0.001 & 0.347 & 0.000 & 0.001 & 0.000 & 0.006 & 0.000 & 0.000 & 0.000 & 0.003 & 0.587 & 0.000 \\
\hline France & 0.000 & 0.070 & 0.329 & 0.000 & 0.002 & 0.000 & 0.001 & 0.000 & 0.000 & 0.000 & 0.161 & 0.931 & 0.000 \\
\hline Italy & 0.000 & 0.104 & 0.355 & 0.012 & 0.000 & 0.000 & 0.000 & 0.000 & 0.000 & 0.000 & 0.042 & 0.316 & 0.000 \\
\hline Spain & 0.000 & 0.134 & 0.896 & 0.004 & 0.011 & 0.000 & 0.000 & 0.000 & 0.004 & 0.000 & 0.101 & 0.102 & 0.000 \\
\hline Austrian & 0.000 & 0.005 & 0.436 & 0.079 & 0.000 & 0.000 & 0.000 & 0.000 & 0.000 & 0.000 & 0.028 & 0.699 & 0.000 \\
\hline SWISS & 0.000 & 0.171 & 0.291 & 0.869 & 0.000 & 0.000 & 0.008 & 0.000 & 0.000 & 0.000 & 0.050 & 0.935 & 0.000 \\
\hline Belgium & 0.000 & 0.048 & 0.536 & 0.000 & 0.000 & 0.000 & 0.000 & 0.000 & 0.000 & 0.000 & 0.023 & 0.821 & 0.000 \\
\hline Denmark & 0.000 & 0.007 & 0.514 & 0.012 & 0.000 & 0.000 & 0.007 & 0.000 & 0.000 & 0.000 & 0.043 & 0.250 & 0.000 \\
\hline Finland & 0.000 & 0.243 & 0.509 & 0.085 & 0.007 & 0.000 & 0.011 & 0.000 & 0.000 & 0.000 & 0.592 & 0.686 & 0.000 \\
\hline Ireland & 0.000 & 0.017 & 0.445 & 0.000 & 0.000 & 0.000 & 0.005 & 0.000 & 0.000 & 0.000 & 0.059 & 0.144 & 0.000 \\
\hline Israel & 0.000 & 0.051 & 0.243 & 0.000 & 0.000 & 0.000 & 0.322 & 0.000 & 0.000 & 0.000 & 0.040 & 0.007 & 0.000 \\
\hline Netherlands & 0.000 & 0.032 & 0.284 & 0.000 & 0.000 & 0.000 & 0.009 & 0.000 & 0.000 & 0.000 & 0.115 & 0.444 & 0.000 \\
\hline Norway & 0.000 & 0.149 & 0.187 & 0.000 & 0.003 & 0.000 & 0.024 & 0.000 & 0.000 & 0.000 & 0.174 & 0.019 & 0.000 \\
\hline Portugal & 0.000 & 0.693 & 0.133 & 0.560 & 0.003 & 0.000 & 0.000 & 0.000 & 0.002 & 0.001 & 0.232 & 0.066 & 0.000 \\
\hline Sweden & 0.000 & 0.049 & 0.283 & 0.001 & 0.012 & 0.000 & 0.048 & 0.000 & 0.000 & 0.001 & 0.339 & 0.322 & 0.000 \\
\hline Czech Rep & 0.000 & 0.016 & 0.375 & 0.000 & 0.000 & 0.000 & 0.000 & 0.000 & 0.000 & 0.000 & 0.006 & 0.443 & 0.000 \\
\hline Greece & 0.000 & 0.550 & 0.957 & 0.000 & 0.992 & 0.010 & 0.154 & 0.011 & 0.806 & 0.081 & 0.575 & 0.025 & 0.000 \\
\hline Hungary & 0.000 & 0.022 & 0.613 & 0.000 & 0.000 & 0.000 & 0.000 & 0.000 & 0.000 & 0.000 & 0.000 & 0.317 & 0.000 \\
\hline Poland & 0.000 & 0.049 & 0.551 & 0.000 & 0.000 & 0.000 & 0.014 & 0.000 & 0.000 & 0.000 & 0.003 & 0.668 & 0.000 \\
\hline Qatar & 0.001 & 0.001 & 0.276 & 0.442 & 0.000 & 0.000 & 0.001 & 0.056 & 0.000 & 0.000 & 0.001 & 0.004 & 0.000 \\
\hline Russian & 0.000 & 0.004 & 0.692 & 0.004 & 0.000 & 0.000 & 0.014 & 0.000 & 0.000 & 0.000 & 0.022 & 0.970 & 0.000 \\
\hline Turkey & 0.000 & 0.871 & 0.927 & 0.000 & 0.000 & 0.005 & 0.014 & 0.000 & 0.000 & 0.000 & 0.001 & 0.206 & 0.000 \\
\hline$U A E$ & 0.000 & 0.004 & 0.101 & 0.198 & 0.000 & 0.000 & 0.001 & 0.058 & 0.000 & 0.010 & 0.100 & 0.027 & 0.000 \\
\hline
\end{tabular}

The table 4 illustrates the result of p-value test of the correlation coefficients between the returns of the capital markets. considering significance level $1 \%$. The main African capital markets. such as South African, Namibia, Egypt, Morocco, Tunisia and Kenya presents significant and positive correlation with European capital markets between periods of data analysis.

The study results suggest that the European investors should look for an African capital markets for an opportunity to maximize their wealth and diversify the investment risk. In the same order, our result contribute to the discussion about the advantage of the International Diversification even if it happens in the African context and furthermore contributes to the literature through application of the Filtered Historical Simulation (FHS) in the optimization portfolio. This methodology, in addition of producing good results, reveals being more cautious in the constitution of investment portfolios than the other methods. However, this model presents lesser returns than others models. 
The result of this study is important for Africa because it can encourage the European investors to transfer part of their financial wealth to Africa by buying assets of African companies, where these companies can to use these financial resources to develop new projects which will pave way in improving the quality of lives of Africans.

Table 5. Efficient portfolios based in the Mean Variance model (MV)

\begin{tabular}{|c|c|c|c|c|c|c|c|c|c|c|}
\hline \multicolumn{11}{|c|}{ Mean Variance model } \\
\hline Portfolios & $\begin{array}{c}\text { Risk } \\
\text { Europe }\end{array}$ & $\begin{array}{l}\text { Return } \\
\text { Europe }\end{array}$ & $\begin{array}{c}\text { Sharpe } \\
\text { Ratio }\end{array}$ & $\begin{array}{c}\text { Downside } \\
\text { Risk } \\
\end{array}$ & $\begin{array}{c}\text { Sortino } \\
\text { Ratio } \\
\end{array}$ & $\begin{array}{c}\text { Risk Europe + } \\
\text { Africa } \\
\end{array}$ & $\begin{array}{c}\text { Return Europe+ } \\
\text { Africa } \\
\end{array}$ & $\begin{array}{c}\text { Sharpe } \\
\text { Ratio }\end{array}$ & $\begin{array}{c}\text { Downside } \\
\text { Risk } \\
\end{array}$ & Sortino Ratio \\
\hline MVP & $2.017 \%$ & $0.083 \%$ & $0.783 \%$ & $1.408 \%$ & $1.122 \%$ & $1.082 \%$ & $0.124 \%$ & $5.205 \%$ & $0.692 \%$ & $8.141 \%$ \\
\hline $\mathbf{P 2}$ & $2.018 \%$ & $0.085 \%$ & $0.891 \%$ & $1.408 \%$ & $1.277 \%$ & $1.083 \%$ & $0.130 \%$ & $5.756 \%$ & $0.689 \%$ & $9.044 \%$ \\
\hline $\mathbf{P 3}$ & $2.018 \%$ & $0.088 \%$ & $0.998 \%$ & $1.407 \%$ & $1.431 \%$ & $1.086 \%$ & $0.136 \%$ & $6.292 \%$ & $0.688 \%$ & $9.933 \%$ \\
\hline $\mathbf{P 4}$ & $2.019 \%$ & $0.090 \%$ & $1.105 \%$ & $1.407 \%$ & $1.585 \%$ & $1.091 \%$ & $0.142 \%$ & $6.811 \%$ & $0.689 \%$ & $10.795 \%$ \\
\hline P5 & $2.019 \%$ & $0.092 \%$ & $1.212 \%$ & $1.407 \%$ & $1.739 \%$ & $1.101 \%$ & $0.148 \%$ & $7.301 \%$ & $0.693 \%$ & $11.601 \%$ \\
\hline P6 & $2.021 \%$ & $0.094 \%$ & $1.318 \%$ & $1.408 \%$ & $1.892 \%$ & $1.116 \%$ & $0.154 \%$ & $7.736 \%$ & $0.700 \%$ & $12.335 \%$ \\
\hline P7 & $2.022 \%$ & $0.096 \%$ & $1.425 \%$ & $1.408 \%$ & $2.046 \%$ & $1.139 \%$ & $0.160 \%$ & $8.112 \%$ & $0.711 \%$ & $12.993 \%$ \\
\hline P8 & $2.024 \%$ & $0.098 \%$ & $1.531 \%$ & $1.409 \%$ & $2.199 \%$ & $1.168 \%$ & $0.166 \%$ & $8.427 \%$ & $0.725 \%$ & $13.572 \%$ \\
\hline P9 & $2.026 \%$ & $0.101 \%$ & $1.636 \%$ & $1.410 \%$ & $2.351 \%$ & $1.202 \%$ & $0.172 \%$ & $8.686 \%$ & $0.742 \%$ & $14.074 \%$ \\
\hline P10 & $2.028 \%$ & $0.103 \%$ & $1.741 \%$ & $1.411 \%$ & $2.503 \%$ & $1.243 \%$ & $0.178 \%$ & $8.881 \%$ & $0.763 \%$ & $14.473 \%$ \\
\hline P11 & $2.030 \%$ & $0.105 \%$ & $1.846 \%$ & $1.412 \%$ & $2.655 \%$ & $1.293 \%$ & $0.184 \%$ & $9.006 \%$ & $0.788 \%$ & $14.767 \%$ \\
\hline P12 & $2.033 \%$ & $0.107 \%$ & $1.950 \%$ & $1.413 \%$ & $2.806 \%$ & $1.349 \%$ & $0.190 \%$ & $9.074 \%$ & $0.818 \%$ & $14.973 \%$ \\
\hline P13 & $2.036 \%$ & $0.109 \%$ & $2.054 \%$ & $1.414 \%$ & $2.956 \%$ & $1.412 \%$ & $0.196 \%$ & $9.095 \%$ & $0.850 \%$ & $15.106 \%$ \\
\hline P14 & $2.039 \%$ & $0.111 \%$ & $2.157 \%$ & $1.416 \%$ & $3.106 \%$ & $1.481 \%$ & $0.202 \%$ & $9.080 \%$ & $0.887 \%$ & $15.160 \%$ \\
\hline P15 & $2.043 \%$ & $0.114 \%$ & $2.259 \%$ & $1.418 \%$ & $3.255 \%$ & $1.555 \%$ & $0.208 \%$ & $9.034 \%$ & $0.927 \%$ & $15.150 \%$ \\
\hline P16 & $2.046 \%$ & $0.116 \%$ & $2.361 \%$ & $1.420 \%$ & $3.404 \%$ & $1.635 \%$ & $0.214 \%$ & $8.962 \%$ & $0.970 \%$ & $15.100 \%$ \\
\hline P17 & $2.050 \%$ & $0.118 \%$ & $2.462 \%$ & $1.422 \%$ & $3.551 \%$ & $1.720 \%$ & $0.220 \%$ & $8.868 \%$ & $1.016 \%$ & $15.016 \%$ \\
\hline P18 & $2.054 \%$ & $0.120 \%$ & $2.563 \%$ & $1.424 \%$ & $3.698 \%$ & $1.809 \%$ & $0.226 \%$ & $8.762 \%$ & $1.063 \%$ & $14.908 \%$ \\
\hline P19 & $2.059 \%$ & $0.122 \%$ & $2.663 \%$ & $1.426 \%$ & $3.844 \%$ & $1.902 \%$ & $0.232 \%$ & $8.648 \%$ & $1.113 \%$ & $14.776 \%$ \\
\hline $\mathbf{P 2 0}$ & $2.064 \%$ & $0.124 \%$ & $2.762 \%$ & $1.429 \%$ & $3.988 \%$ & $1.999 \%$ & $0.238 \%$ & $8.530 \%$ & $1.165 \%$ & $14.632 \%$ \\
\hline $\mathbf{P 2 1}$ & $2.068 \%$ & $0.127 \%$ & $2.860 \%$ & $1.432 \%$ & $4.132 \%$ & $2.099 \%$ & $0.244 \%$ & $8.410 \%$ & $1.219 \%$ & $14.483 \%$ \\
\hline P22 & $2.074 \%$ & $0.129 \%$ & $2.957 \%$ & $1.435 \%$ & $4.272 \%$ & $2.202 \%$ & $0.250 \%$ & $8.290 \%$ & $1.272 \%$ & $14.356 \%$ \\
\hline $\mathbf{P 2 3}$ & $2.080 \%$ & $0.131 \%$ & $3.053 \%$ & $1.440 \%$ & $4.410 \%$ & $2.310 \%$ & $0.256 \%$ & $8.164 \%$ & $1.325 \%$ & $14.232 \%$ \\
\hline P24 & $2.087 \%$ & $0.133 \%$ & $3.147 \%$ & $1.445 \%$ & $4.545 \%$ & $2.421 \%$ & $0.262 \%$ & $8.037 \%$ & $1.380 \%$ & $14.101 \%$ \\
\hline P25 & $2.094 \%$ & $0.135 \%$ & $3.239 \%$ & $1.450 \%$ & $4.678 \%$ & $2.536 \%$ & $0.268 \%$ & $7.910 \%$ & $1.436 \%$ & $13.964 \%$ \\
\hline P26 & $2.103 \%$ & $0.137 \%$ & $3.329 \%$ & $1.456 \%$ & $4.808 \%$ & $2.654 \%$ & $0.274 \%$ & $7.785 \%$ & $1.494 \%$ & $13.826 \%$ \\
\hline P27 & $2.112 \%$ & $0.140 \%$ & $3.417 \%$ & $1.462 \%$ & $4.935 \%$ & $2.774 \%$ & $0.280 \%$ & $7.664 \%$ & $1.553 \%$ & $13.688 \%$ \\
\hline P28 & $2.122 \%$ & $0.142 \%$ & $3.503 \%$ & $1.469 \%$ & $5.059 \%$ & $2.897 \%$ & $0.286 \%$ & $7.546 \%$ & $1.613 \%$ & $13.556 \%$ \\
\hline P29 & $2.132 \%$ & $0.144 \%$ & $3.587 \%$ & $1.477 \%$ & $5.181 \%$ & $3.024 \%$ & $0.292 \%$ & $7.429 \%$ & $1.654 \%$ & $13.584 \%$ \\
\hline P30 & $2.144 \%$ & $0.146 \%$ & $3.670 \%$ & $1.485 \%$ & $5.299 \%$ & $3.156 \%$ & $0.298 \%$ & $7.307 \%$ & $1.698 \%$ & $13.587 \%$ \\
\hline P31 & $2.156 \%$ & $0.148 \%$ & $3.750 \%$ & $1.493 \%$ & $5.414 \%$ & $3.294 \%$ & $0.304 \%$ & $7.185 \%$ & $1.744 \%$ & $13.568 \%$ \\
\hline P32 & $2.169 \%$ & $0.151 \%$ & $3.828 \%$ & $1.502 \%$ & $5.527 \%$ & $3.435 \%$ & $0.310 \%$ & $7.064 \%$ & $1.793 \%$ & $13.533 \%$ \\
\hline P33 & $2.182 \%$ & $0.153 \%$ & $3.903 \%$ & $1.511 \%$ & $5.636 \%$ & $3.581 \%$ & $0.316 \%$ & $6.945 \%$ & $1.844 \%$ & $13.485 \%$ \\
\hline P34 & $2.196 \%$ & $0.155 \%$ & $3.977 \%$ & $1.521 \%$ & $5.743 \%$ & $3.730 \%$ & $0.322 \%$ & $6.829 \%$ & $1.897 \%$ & $13.425 \%$ \\
\hline P35 & $2.211 \%$ & $0.157 \%$ & $4.049 \%$ & $1.531 \%$ & $5.848 \%$ & $3.882 \%$ & $0.328 \%$ & $6.716 \%$ & $1.952 \%$ & $13.354 \%$ \\
\hline P36 & $2.226 \%$ & $0.159 \%$ & $4.118 \%$ & $1.541 \%$ & $5.950 \%$ & $4.038 \%$ & $0.334 \%$ & $6.605 \%$ & $1.998 \%$ & $13.347 \%$ \\
\hline P37 & $2.242 \%$ & $0.161 \%$ & $4.185 \%$ & $1.552 \%$ & $6.048 \%$ & $4.202 \%$ & $0.340 \%$ & $6.491 \%$ & $2.047 \%$ & $13.327 \%$ \\
\hline P38 & $2.259 \%$ & $0.164 \%$ & $4.250 \%$ & $1.563 \%$ & $6.143 \%$ & $4.371 \%$ & $0.346 \%$ & $6.377 \%$ & $2.098 \%$ & $13.286 \%$ \\
\hline P39 & $2.277 \%$ & $0.166 \%$ & $4.312 \%$ & $1.574 \%$ & $6.236 \%$ & $4.546 \%$ & $0.352 \%$ & $6.264 \%$ & $2.153 \%$ & $13.228 \%$ \\
\hline P40 & $2.296 \%$ & $0.168 \%$ & $4.371 \%$ & $1.582 \%$ & $6.342 \%$ & $4.726 \%$ & $0.358 \%$ & $6.153 \%$ & $2.210 \%$ & $13.155 \%$ \\
\hline P41 & $2.321 \%$ & $0.170 \%$ & $4.416 \%$ & $1.589 \%$ & $6.451 \%$ & $4.910 \%$ & $0.364 \%$ & $6.045 \%$ & $2.271 \%$ & $13.070 \%$ \\
\hline P42 & $2.354 \%$ & $0.172 \%$ & $4.447 \%$ & $1.600 \%$ & $6.544 \%$ & $5.097 \%$ & $0.370 \%$ & $5.941 \%$ & $2.334 \%$ & $12.976 \%$ \\
\hline $\mathbf{P 4 3}$ & $2.394 \%$ & $0.174 \%$ & $4.464 \%$ & $1.614 \%$ & $6.620 \%$ & $5.288 \%$ & $0.376 \%$ & $5.840 \%$ & $2.399 \%$ & $12.874 \%$ \\
\hline P44 & $2.440 \%$ & $0.177 \%$ & $4.469 \%$ & $1.632 \%$ & $6.681 \%$ & $5.482 \%$ & $0.382 \%$ & $5.743 \%$ & $2.466 \%$ & $12.767 \%$ \\
\hline P45 & $2.492 \%$ & $0.179 \%$ & $4.462 \%$ & $1.653 \%$ & $6.727 \%$ & $5.679 \%$ & $0.388 \%$ & $5.650 \%$ & $2.535 \%$ & $12.656 \%$ \\
\hline P46 & $2.550 \%$ & $0.181 \%$ & $4.446 \%$ & $1.677 \%$ & $6.758 \%$ & $5.878 \%$ & $0.394 \%$ & $5.560 \%$ & $2.606 \%$ & $12.542 \%$ \\
\hline P47 & $2.613 \%$ & $0.183 \%$ & $4.421 \%$ & $1.705 \%$ & $6.776 \%$ & $6.080 \%$ & $0.400 \%$ & $5.475 \%$ & $2.679 \%$ & $12.426 \%$ \\
\hline P48 & $2.681 \%$ & $0.185 \%$ & $4.390 \%$ & $1.735 \%$ & $6.781 \%$ & $6.283 \%$ & $0.406 \%$ & $5.394 \%$ & $2.753 \%$ & $12.309 \%$ \\
\hline P49 & $2.837 \%$ & $0.187 \%$ & $4.225 \%$ & $1.846 \%$ & $6.494 \%$ & $6.488 \%$ & $0.412 \%$ & $5.316 \%$ & $2.829 \%$ & $12.193 \%$ \\
\hline . & $3.266 \%$ & $0.190 \%$ & $3.736 \%$ & $2.147 \%$ & $5.683 \%$ & $6.695 \%$ & $0.418 \%$ & $5.241 \%$ & $2.905 \%$ & $12.077 \%$ \\
\hline
\end{tabular}

Source: author. 
Table 6. Efficient portfolios based in the Resample Michaud model (RM)

\begin{tabular}{|c|c|c|c|c|c|c|c|c|c|c|}
\hline \multicolumn{11}{|c|}{ Resample Michaud model } \\
\hline Portfolios & $\begin{array}{c}\text { Risk } \\
\text { Europe }\end{array}$ & $\begin{array}{l}\text { Return } \\
\text { Europe }\end{array}$ & $\begin{array}{c}\text { Sharpe } \\
\text { Ratio }\end{array}$ & $\begin{array}{c}\text { Downside } \\
\text { Risk }\end{array}$ & $\begin{array}{c}\text { Sortino } \\
\text { Ratio }\end{array}$ & $\begin{array}{c}\text { Risk Europe + } \\
\text { Africa }\end{array}$ & $\begin{array}{c}\text { Return Europe+ } \\
\text { Africa } \\
\end{array}$ & $\begin{array}{c}\text { Sharpe } \\
\text { Ratio } \\
\end{array}$ & $\begin{array}{c}\text { Downside } \\
\text { Risk }\end{array}$ & $\begin{array}{c}\text { Sortino } \\
\text { Ratio }\end{array}$ \\
\hline MVP & $2.135 \%$ & $0.067 \%$ & $0.000 \%$ & $1.195 \%$ & $0.000 \%$ & $1.029 \%$ & $0.142 \%$ & $7.277 \%$ & $0.554 \%$ & $13.515 \%$ \\
\hline P2 & $2.151 \%$ & $0.071 \%$ & $0.164 \%$ & $1.205 \%$ & $0.293 \%$ & $1.031 \%$ & $0.160 \%$ & $8.990 \%$ & $0.555 \%$ & $16.694 \%$ \\
\hline P3 & $2.167 \%$ & $0.075 \%$ & $0.326 \%$ & $1.215 \%$ & $0.582 \%$ & $1.039 \%$ & $0.178 \%$ & $10.636 \%$ & $0.559 \%$ & $19.761 \%$ \\
\hline P4 & $2.185 \%$ & $0.078 \%$ & $0.485 \%$ & $1.226 \%$ & $0.865 \%$ & $1.054 \%$ & $0.196 \%$ & $12.185 \%$ & $0.567 \%$ & $22.660 \%$ \\
\hline P5 & $2.205 \%$ & $0.082 \%$ & $0.641 \%$ & $1.238 \%$ & $1.142 \%$ & $1.074 \%$ & $0.214 \%$ & $13.611 \%$ & $0.578 \%$ & $25.316 \%$ \\
\hline P6 & $2.226 \%$ & $0.085 \%$ & $0.794 \%$ & $1.250 \%$ & $1.414 \%$ & $1.102 \%$ & $0.232 \%$ & $14.888 \%$ & $0.593 \%$ & $27.678 \%$ \\
\hline P7 & $2.249 \%$ & $0.089 \%$ & $0.943 \%$ & $1.262 \%$ & $1.680 \%$ & $1.138 \%$ & $0.249 \%$ & $15.989 \%$ & $0.611 \%$ & $29.750 \%$ \\
\hline P8 & $2.274 \%$ & $0.092 \%$ & $1.088 \%$ & $1.276 \%$ & $1.940 \%$ & $1.181 \%$ & $0.267 \%$ & $16.908 \%$ & $0.634 \%$ & $31.491 \%$ \\
\hline P9 & $2.300 \%$ & $0.096 \%$ & $1.230 \%$ & $1.290 \%$ & $2.192 \%$ & $1.234 \%$ & $0.285 \%$ & $17.636 \%$ & $0.659 \%$ & $32.997 \%$ \\
\hline P10 & $2.329 \%$ & $0.099 \%$ & $1.366 \%$ & $1.306 \%$ & $2.436 \%$ & $1.294 \%$ & $0.303 \%$ & $18.188 \%$ & $0.689 \%$ & $34.159 \%$ \\
\hline P11 & $2.359 \%$ & $0.103 \%$ & $1.499 \%$ & $1.323 \%$ & $2.673 \%$ & $1.362 \%$ & $0.321 \%$ & $18.591 \%$ & $0.723 \%$ & $35.025 \%$ \\
\hline P12 & $2.390 \%$ & $0.106 \%$ & $1.627 \%$ & $1.340 \%$ & $2.903 \%$ & $1.436 \%$ & $0.339 \%$ & $18.875 \%$ & $0.761 \%$ & $35.646 \%$ \\
\hline P13 & $2.424 \%$ & $0.110 \%$ & $1.750 \%$ & $1.357 \%$ & $3.126 \%$ & $1.516 \%$ & $0.356 \%$ & $19.063 \%$ & $0.801 \%$ & $36.071 \%$ \\
\hline P14 & $2.459 \%$ & $0.113 \%$ & $1.869 \%$ & $1.376 \%$ & $3.341 \%$ & $1.600 \%$ & $0.374 \%$ & $19.179 \%$ & $0.844 \%$ & $36.343 \%$ \\
\hline P15 & $2.496 \%$ & $0.117 \%$ & $1.983 \%$ & $1.395 \%$ & $3.547 \%$ & $1.688 \%$ & $0.392 \%$ & $19.237 \%$ & $0.889 \%$ & $36.513 \%$ \\
\hline P16 & $2.535 \%$ & $0.121 \%$ & $2.092 \%$ & $1.416 \%$ & $3.744 \%$ & $1.780 \%$ & $0.410 \%$ & $19.240 \%$ & $0.936 \%$ & $36.577 \%$ \\
\hline P17 & $2.576 \%$ & $0.124 \%$ & $2.196 \%$ & $1.438 \%$ & $3.933 \%$ & $1.876 \%$ & $0.428 \%$ & $19.202 \%$ & $0.986 \%$ & $36.542 \%$ \\
\hline P18 & $2.618 \%$ & $0.128 \%$ & $2.296 \%$ & $1.461 \%$ & $4.113 \%$ & $1.976 \%$ & $0.446 \%$ & $19.133 \%$ & $1.038 \%$ & $36.445 \%$ \\
\hline P19 & $2.661 \%$ & $0.131 \%$ & $2.391 \%$ & $1.485 \%$ & $4.285 \%$ & $2.079 \%$ & $0.463 \%$ & $19.044 \%$ & $1.091 \%$ & $36.296 \%$ \\
\hline P20 & $2.706 \%$ & $0.135 \%$ & $2.482 \%$ & $1.510 \%$ & $4.448 \%$ & $2.185 \%$ & $0.481 \%$ & $18.940 \%$ & $1.146 \%$ & $36.109 \%$ \\
\hline P21 & $2.753 \%$ & $0.138 \%$ & $2.569 \%$ & $1.536 \%$ & $4.604 \%$ & $2.293 \%$ & $0.499 \%$ & $18.826 \%$ & $1.202 \%$ & $35.900 \%$ \\
\hline P22 & $2.800 \%$ & $0.142 \%$ & $2.651 \%$ & $1.563 \%$ & $4.750 \%$ & $2.404 \%$ & $0.517 \%$ & $18.702 \%$ & $1.260 \%$ & $35.680 \%$ \\
\hline $\mathbf{P 2 3}$ & $2.852 \%$ & $0.145 \%$ & $2.728 \%$ & $1.592 \%$ & $4.885 \%$ & $2.517 \%$ & $0.535 \%$ & $18.566 \%$ & $1.319 \%$ & $35.434 \%$ \\
\hline P24 & $2.906 \%$ & $0.149 \%$ & $2.798 \%$ & $1.624 \%$ & $5.007 \%$ & $2.633 \%$ & $0.553 \%$ & $18.425 \%$ & $1.380 \%$ & $35.171 \%$ \\
\hline P25 & $2.964 \%$ & $0.152 \%$ & $2.863 \%$ & $1.658 \%$ & $5.119 \%$ & $2.752 \%$ & $0.571 \%$ & $18.280 \%$ & $1.441 \%$ & $34.903 \%$ \\
\hline P26 & $3.025 \%$ & $0.156 \%$ & $2.922 \%$ & $1.693 \%$ & $5.220 \%$ & $2.876 \%$ & $0.588 \%$ & $18.111 \%$ & $1.505 \%$ & $34.603 \%$ \\
\hline P27 & $3.088 \%$ & $0.159 \%$ & $2.976 \%$ & $1.731 \%$ & $5.310 \%$ & $3.007 \%$ & $0.606 \%$ & $17.917 \%$ & $1.573 \%$ & $34.252 \%$ \\
\hline P28 & $3.154 \%$ & $0.163 \%$ & $3.026 \%$ & $1.770 \%$ & $5.392 \%$ & $3.143 \%$ & $0.624 \%$ & $17.708 \%$ & $1.643 \%$ & $33.866 \%$ \\
\hline P29 & $3.223 \%$ & $0.166 \%$ & $3.071 \%$ & $1.811 \%$ & $5.465 \%$ & $3.284 \%$ & $0.642 \%$ & $17.489 \%$ & $1.717 \%$ & $33.459 \%$ \\
\hline P30 & $3.294 \%$ & $0.170 \%$ & $3.112 \%$ & $1.854 \%$ & $5.531 \%$ & $3.430 \%$ & $0.660 \%$ & $17.265 \%$ & $1.792 \%$ & $33.042 \%$ \\
\hline P31 & $3.368 \%$ & $0.174 \%$ & $3.149 \%$ & $1.898 \%$ & $5.589 \%$ & $3.580 \%$ & $0.678 \%$ & $17.042 \%$ & $1.870 \%$ & $32.622 \%$ \\
\hline P32 & $3.443 \%$ & $0.177 \%$ & $3.183 \%$ & $1.943 \%$ & $5.641 \%$ & $3.733 \%$ & $0.695 \%$ & $16.822 \%$ & $1.950 \%$ & $32.205 \%$ \\
\hline P33 & $3.520 \%$ & $0.181 \%$ & $3.214 \%$ & $1.989 \%$ & $5.687 \%$ & $3.889 \%$ & $0.713 \%$ & $16.606 \%$ & $2.031 \%$ & $31.796 \%$ \\
\hline P34 & $3.599 \%$ & $0.184 \%$ & $3.241 \%$ & $2.037 \%$ & $5.728 \%$ & $4.047 \%$ & $0.731 \%$ & $16.396 \%$ & $2.114 \%$ & $31.397 \%$ \\
\hline P35 & $3.680 \%$ & $0.188 \%$ & $3.266 \%$ & $2.086 \%$ & $5.764 \%$ & $4.208 \%$ & $0.749 \%$ & $16.193 \%$ & $2.198 \%$ & $31.010 \%$ \\
\hline P36 & $3.763 \%$ & $0.191 \%$ & $3.289 \%$ & $2.135 \%$ & $5.795 \%$ & $4.371 \%$ & $0.767 \%$ & $15.997 \%$ & $2.283 \%$ & $30.636 \%$ \\
\hline P37 & $3.847 \%$ & $0.195 \%$ & $3.309 \%$ & $2.186 \%$ & $5.823 \%$ & $4.536 \%$ & $0.785 \%$ & $15.809 \%$ & $2.369 \%$ & $30.277 \%$ \\
\hline P38 & $3.932 \%$ & $0.198 \%$ & $3.327 \%$ & $2.237 \%$ & $5.847 \%$ & $4.703 \%$ & $0.802 \%$ & $15.628 \%$ & $2.456 \%$ & $29.931 \%$ \\
\hline P39 & $4.018 \%$ & $0.202 \%$ & $3.343 \%$ & $2.290 \%$ & $5.868 \%$ & $4.871 \%$ & $0.820 \%$ & $15.455 \%$ & $2.543 \%$ & $29.600 \%$ \\
\hline P40 & $4.106 \%$ & $0.205 \%$ & $3.358 \%$ & $2.343 \%$ & $5.885 \%$ & $5.040 \%$ & $0.838 \%$ & $15.290 \%$ & $2.632 \%$ & $29.283 \%$ \\
\hline P41 & $4.195 \%$ & $0.209 \%$ & $3.371 \%$ & $2.398 \%$ & $5.897 \%$ & $5.211 \%$ & $0.856 \%$ & $15.131 \%$ & $2.721 \%$ & $28.979 \%$ \\
\hline P42 & $4.288 \%$ & $0.212 \%$ & $3.381 \%$ & $2.455 \%$ & $5.904 \%$ & $5.383 \%$ & $0.874 \%$ & $14.980 \%$ & $2.811 \%$ & $28.689 \%$ \\
\hline P43 & $4.382 \%$ & $0.216 \%$ & $3.388 \%$ & $2.514 \%$ & $5.906 \%$ & $5.555 \%$ & $0.892 \%$ & $14.836 \%$ & $2.901 \%$ & $28.411 \%$ \\
\hline P44 & $4.480 \%$ & $0.220 \%$ & $3.393 \%$ & $2.574 \%$ & $5.905 \%$ & $5.729 \%$ & $0.910 \%$ & $14.698 \%$ & $2.992 \%$ & $28.146 \%$ \\
\hline P45 & $4.580 \%$ & $0.223 \%$ & $3.397 \%$ & $2.636 \%$ & $5.901 \%$ & $5.903 \%$ & $0.927 \%$ & $14.565 \%$ & $3.083 \%$ & $27.892 \%$ \\
\hline P46 & $4.682 \%$ & $0.227 \%$ & $3.398 \%$ & $2.699 \%$ & $5.894 \%$ & $6.079 \%$ & $0.945 \%$ & $14.439 \%$ & $3.174 \%$ & $27.649 \%$ \\
\hline P47 & $4.786 \%$ & $0.230 \%$ & $3.398 \%$ & $2.764 \%$ & $5.885 \%$ & $6.255 \%$ & $0.963 \%$ & $14.318 \%$ & $3.266 \%$ & $27.417 \%$ \\
\hline P48 & $4.892 \%$ & $0.234 \%$ & $3.397 \%$ & $2.829 \%$ & $5.873 \%$ & $6.431 \%$ & $0.981 \%$ & $14.203 \%$ & $3.359 \%$ & $27.194 \%$ \\
\hline P49 & $5.000 \%$ & $0.237 \%$ & $3.394 \%$ & $2.896 \%$ & $5.860 \%$ & $6.608 \%$ & $0.999 \%$ & $14.092 \%$ & $3.451 \%$ & $26.981 \%$ \\
\hline P50 & $5.110 \%$ & $0.241 \%$ & $3.390 \%$ & $2.963 \%$ & $5.846 \%$ & $6.786 \%$ & $1.017 \%$ & $13.986 \%$ & $3.544 \%$ & $26.777 \%$ \\
\hline
\end{tabular}

Source: author. 
Table 7. Efficient portfolios based in the SemiVariance model (SV)

\begin{tabular}{|c|c|c|c|c|c|c|c|c|c|c|}
\hline \multicolumn{11}{|c|}{ SemiVariance model } \\
\hline Portfolios & $\begin{array}{c}\text { Risk } \\
\text { Europe }\end{array}$ & $\begin{array}{l}\text { Return } \\
\text { Europe }\end{array}$ & $\begin{array}{c}\text { Sharpe } \\
\text { Ratio }\end{array}$ & $\begin{array}{c}\text { Downside } \\
\text { Risk }\end{array}$ & $\begin{array}{c}\text { Sortino } \\
\text { Ratio }\end{array}$ & $\begin{array}{c}\text { Risk Europe + } \\
\text { Africa }\end{array}$ & $\begin{array}{c}\text { Return Europe+ } \\
\text { Africa }\end{array}$ & $\begin{array}{c}\text { Sharpe } \\
\text { Ratio }\end{array}$ & $\begin{array}{c}\text { Downside } \\
\text { Risk } \\
\end{array}$ & $\begin{array}{c}\text { Sortino } \\
\text { Ratio } \\
\end{array}$ \\
\hline MVP & $1.398 \%$ & $0.089 \%$ & $1.533 \%$ & $1.398 \%$ & $1.533 \%$ & $0.658 \%$ & $0.132 \%$ & $9.777 \%$ & $0.658 \%$ & $9.777 \%$ \\
\hline $\mathbf{P 2}$ & $1.398 \%$ & $0.091 \%$ & $1.680 \%$ & $1.398 \%$ & $1.680 \%$ & $0.659 \%$ & $0.138 \%$ & $10.657 \%$ & $0.659 \%$ & $10.657 \%$ \\
\hline $\mathbf{P 3}$ & $1.398 \%$ & $0.093 \%$ & $1.827 \%$ & $1.398 \%$ & $1.827 \%$ & $0.660 \%$ & $0.144 \%$ & $11.512 \%$ & $0.660 \%$ & $11.512 \%$ \\
\hline P4 & $1.399 \%$ & $0.095 \%$ & $1.973 \%$ & $1.399 \%$ & $1.973 \%$ & $0.665 \%$ & $0.149 \%$ & $12.317 \%$ & $0.665 \%$ & $12.317 \%$ \\
\hline P5 & $1.399 \%$ & $0.097 \%$ & $2.119 \%$ & $1.399 \%$ & $2.119 \%$ & $0.672 \%$ & $0.155 \%$ & $13.055 \%$ & $0.672 \%$ & $13.055 \%$ \\
\hline P6 & $1.400 \%$ & $0.099 \%$ & $2.265 \%$ & $1.400 \%$ & $2.265 \%$ & $0.682 \%$ & $0.161 \%$ & $13.719 \%$ & $0.682 \%$ & $13.719 \%$ \\
\hline P7 & $1.401 \%$ & $0.101 \%$ & $2.410 \%$ & $1.401 \%$ & $2.410 \%$ & $0.695 \%$ & $0.167 \%$ & $14.308 \%$ & $0.695 \%$ & $14.308 \%$ \\
\hline P8 & $1.402 \%$ & $0.103 \%$ & $2.555 \%$ & $1.402 \%$ & $2.555 \%$ & $0.710 \%$ & $0.173 \%$ & $14.821 \%$ & $0.710 \%$ & $14.821 \%$ \\
\hline P9 & $1.403 \%$ & $0.105 \%$ & $2.699 \%$ & $1.403 \%$ & $2.699 \%$ & $0.729 \%$ & $0.179 \%$ & $15.246 \%$ & $0.729 \%$ & $15.246 \%$ \\
\hline P10 & $1.404 \%$ & $0.107 \%$ & $2.843 \%$ & $1.404 \%$ & $2.843 \%$ & $0.751 \%$ & $0.184 \%$ & $15.573 \%$ & $0.751 \%$ & $15.573 \%$ \\
\hline P11 & $1.405 \%$ & $0.109 \%$ & $2.986 \%$ & $1.405 \%$ & $2.986 \%$ & $0.777 \%$ & $0.190 \%$ & $15.808 \%$ & $0.777 \%$ & $15.808 \%$ \\
\hline P12 & $1.407 \%$ & $0.112 \%$ & $3.129 \%$ & $1.407 \%$ & $3.129 \%$ & $0.806 \%$ & $0.196 \%$ & $15.964 \%$ & $0.806 \%$ & $15.964 \%$ \\
\hline P13 & $1.409 \%$ & $0.114 \%$ & $3.271 \%$ & $1.409 \%$ & $3.271 \%$ & $0.838 \%$ & $0.202 \%$ & $16.055 \%$ & $0.838 \%$ & $16.055 \%$ \\
\hline P14 & $1.410 \%$ & $0.116 \%$ & $3.412 \%$ & $1.410 \%$ & $3.412 \%$ & $0.872 \%$ & $0.208 \%$ & $16.092 \%$ & $0.872 \%$ & $16.092 \%$ \\
\hline P15 & $1.412 \%$ & $0.118 \%$ & $3.553 \%$ & $1.412 \%$ & $3.553 \%$ & $0.909 \%$ & $0.214 \%$ & $16.087 \%$ & $0.909 \%$ & $16.087 \%$ \\
\hline P16 & $1.415 \%$ & $0.120 \%$ & $3.692 \%$ & $1.415 \%$ & $3.692 \%$ & $0.948 \%$ & $0.220 \%$ & $16.048 \%$ & $0.948 \%$ & $16.048 \%$ \\
\hline P17 & $1.417 \%$ & $0.122 \%$ & $3.831 \%$ & $1.417 \%$ & $3.831 \%$ & $0.988 \%$ & $0.225 \%$ & $15.977 \%$ & $0.988 \%$ & $15.977 \%$ \\
\hline P18 & $1.419 \%$ & $0.124 \%$ & $3.970 \%$ & $1.419 \%$ & $3.970 \%$ & $1.031 \%$ & $0.231 \%$ & $15.881 \%$ & $1.031 \%$ & $15.881 \%$ \\
\hline P19 & $1.422 \%$ & $0.126 \%$ & $4.107 \%$ & $1.422 \%$ & $4.107 \%$ & $1.076 \%$ & $0.237 \%$ & $15.769 \%$ & $1.076 \%$ & $15.769 \%$ \\
\hline P20 & $1.425 \%$ & $0.128 \%$ & $4.243 \%$ & $1.425 \%$ & $4.243 \%$ & $1.121 \%$ & $0.243 \%$ & $15.645 \%$ & $1.121 \%$ & $15.645 \%$ \\
\hline P21 & $1.428 \%$ & $0.130 \%$ & $4.376 \%$ & $1.428 \%$ & $4.376 \%$ & $1.169 \%$ & $0.249 \%$ & $15.512 \%$ & $1.169 \%$ & $15.512 \%$ \\
\hline P22 & $1.432 \%$ & $0.132 \%$ & $4.507 \%$ & $1.432 \%$ & $4.507 \%$ & $1.217 \%$ & $0.255 \%$ & $15.376 \%$ & $1.217 \%$ & $15.376 \%$ \\
\hline $\mathbf{P 2 3}$ & $1.437 \%$ & $0.134 \%$ & $4.635 \%$ & $1.437 \%$ & $4.635 \%$ & $1.267 \%$ & $0.260 \%$ & $15.238 \%$ & $1.267 \%$ & $15.238 \%$ \\
\hline P24 & $1.442 \%$ & $0.136 \%$ & $4.762 \%$ & $1.442 \%$ & $4.762 \%$ & $1.317 \%$ & $0.266 \%$ & $15.099 \%$ & $1.317 \%$ & $15.099 \%$ \\
\hline P25 & $1.447 \%$ & $0.138 \%$ & $4.886 \%$ & $1.447 \%$ & $4.886 \%$ & $1.368 \%$ & $0.272 \%$ & $14.962 \%$ & $1.368 \%$ & $14.962 \%$ \\
\hline P26 & $1.453 \%$ & $0.140 \%$ & $5.007 \%$ & $1.453 \%$ & $5.007 \%$ & $1.420 \%$ & $0.278 \%$ & $14.826 \%$ & $1.420 \%$ & $14.826 \%$ \\
\hline P27 & $1.459 \%$ & $0.142 \%$ & $5.126 \%$ & $1.459 \%$ & $5.126 \%$ & $1.473 \%$ & $0.284 \%$ & $14.690 \%$ & $1.473 \%$ & $14.690 \%$ \\
\hline P28 & $1.466 \%$ & $0.144 \%$ & $5.242 \%$ & $1.466 \%$ & $5.242 \%$ & $1.527 \%$ & $0.290 \%$ & $14.553 \%$ & $1.527 \%$ & $14.553 \%$ \\
\hline P29 & $1.473 \%$ & $0.146 \%$ & $5.356 \%$ & $1.473 \%$ & $5.356 \%$ & $1.582 \%$ & $0.296 \%$ & $14.418 \%$ & $1.582 \%$ & $14.418 \%$ \\
\hline P30 & $1.481 \%$ & $0.148 \%$ & $5.467 \%$ & $1.481 \%$ & $5.467 \%$ & $1.638 \%$ & $0.301 \%$ & $14.285 \%$ & $1.638 \%$ & $14.285 \%$ \\
\hline P31 & $1.489 \%$ & $0.151 \%$ & $5.575 \%$ & $1.489 \%$ & $5.575 \%$ & $1.694 \%$ & $0.307 \%$ & $14.155 \%$ & $1.694 \%$ & $14.155 \%$ \\
\hline P32 & $1.498 \%$ & $0.153 \%$ & $5.681 \%$ & $1.498 \%$ & $5.681 \%$ & $1.751 \%$ & $0.313 \%$ & $14.028 \%$ & $1.751 \%$ & $14.028 \%$ \\
\hline P33 & $1.506 \%$ & $0.155 \%$ & $5.784 \%$ & $1.506 \%$ & $5.784 \%$ & $1.809 \%$ & $0.319 \%$ & $13.904 \%$ & $1.809 \%$ & $13.904 \%$ \\
\hline P34 & $1.516 \%$ & $0.157 \%$ & $5.884 \%$ & $1.516 \%$ & $5.884 \%$ & $1.867 \%$ & $0.325 \%$ & $13.784 \%$ & $1.867 \%$ & $13.784 \%$ \\
\hline P35 & $1.525 \%$ & $0.159 \%$ & $5.981 \%$ & $1.525 \%$ & $5.981 \%$ & $1.925 \%$ & $0.331 \%$ & $13.668 \%$ & $1.925 \%$ & $13.668 \%$ \\
\hline P36 & $1.535 \%$ & $0.161 \%$ & $6.075 \%$ & $1.535 \%$ & $6.075 \%$ & $1.985 \%$ & $0.337 \%$ & $13.556 \%$ & $1.985 \%$ & $13.556 \%$ \\
\hline P37 & $1.546 \%$ & $0.163 \%$ & $6.167 \%$ & $1.546 \%$ & $6.167 \%$ & $2.044 \%$ & $0.342 \%$ & $13.447 \%$ & $2.044 \%$ & $13.447 \%$ \\
\hline P38 & $1.557 \%$ & $0.165 \%$ & $6.256 \%$ & $1.557 \%$ & $6.256 \%$ & $2.104 \%$ & $0.348 \%$ & $13.343 \%$ & $2.104 \%$ & $13.343 \%$ \\
\hline P39 & $1.568 \%$ & $0.167 \%$ & $6.342 \%$ & $1.568 \%$ & $6.342 \%$ & $2.164 \%$ & $0.354 \%$ & $13.241 \%$ & $2.164 \%$ & $13.241 \%$ \\
\hline P40 & $1.579 \%$ & $0.169 \%$ & $6.426 \%$ & $1.579 \%$ & $6.426 \%$ & $2.225 \%$ & $0.360 \%$ & $13.143 \%$ & $2.225 \%$ & $13.143 \%$ \\
\hline P41 & $1.591 \%$ & $0.171 \%$ & $6.507 \%$ & $1.591 \%$ & $6.507 \%$ & $2.286 \%$ & $0.366 \%$ & $13.049 \%$ & $2.286 \%$ & $13.049 \%$ \\
\hline P42 & $1.604 \%$ & $0.173 \%$ & $6.584 \%$ & $1.604 \%$ & $6.584 \%$ & $2.348 \%$ & $0.372 \%$ & $12.955 \%$ & $2.348 \%$ & $12.955 \%$ \\
\hline P43 & $1.619 \%$ & $0.175 \%$ & $6.651 \%$ & $1.619 \%$ & $6.651 \%$ & $2.411 \%$ & $0.377 \%$ & $12.854 \%$ & $2.411 \%$ & $12.854 \%$ \\
\hline P44 & $1.637 \%$ & $0.177 \%$ & $6.704 \%$ & $1.637 \%$ & $6.704 \%$ & $2.477 \%$ & $0.383 \%$ & $12.749 \%$ & $2.477 \%$ & $12.749 \%$ \\
\hline P45 & $1.657 \%$ & $0.179 \%$ & $6.743 \%$ & $1.657 \%$ & $6.743 \%$ & $2.545 \%$ & $0.389 \%$ & $12.640 \%$ & $2.545 \%$ & $12.640 \%$ \\
\hline P46 & $1.681 \%$ & $0.181 \%$ & $6.770 \%$ & $1.681 \%$ & $6.770 \%$ & $2.614 \%$ & $0.395 \%$ & $12.529 \%$ & $2.614 \%$ & $12.529 \%$ \\
\hline P47 & $1.708 \%$ & $0.183 \%$ & $6.785 \%$ & $1.708 \%$ & $6.785 \%$ & $2.685 \%$ & $0.401 \%$ & $12.416 \%$ & $2.685 \%$ & $12.416 \%$ \\
\hline P48 & $1.737 \%$ & $0.185 \%$ & $6.789 \%$ & $1.737 \%$ & $6.789 \%$ & $2.757 \%$ & $0.407 \%$ & $12.303 \%$ & $2.757 \%$ & $12.303 \%$ \\
\hline P49 & $1.857 \%$ & $0.187 \%$ & $6.459 \%$ & $1.857 \%$ & $6.459 \%$ & $2.831 \%$ & $0.413 \%$ & $12.190 \%$ & $2.831 \%$ & $12.190 \%$ \\
\hline P50 & $2.147 \%$ & $0.190 \%$ & $5.683 \%$ & $2.147 \%$ & $5.683 \%$ & $2.905 \%$ & $0.418 \%$ & $12.077 \%$ & $2.905 \%$ & $12.077 \%$ \\
\hline
\end{tabular}

Source: author. 
Table 8. Efficient portfolios based in the Mean Absolute Deviation (MAD)

\begin{tabular}{|c|c|c|c|c|c|c|c|c|c|c|}
\hline \multicolumn{11}{|c|}{ Mean Absolute Deviation model } \\
\hline Portfolios & $\begin{array}{c}\text { Risk } \\
\text { Europe }\end{array}$ & $\begin{array}{l}\text { Return } \\
\text { Europe }\end{array}$ & $\begin{array}{c}\text { Sharpe } \\
\text { Ratio }\end{array}$ & $\begin{array}{c}\text { Downside } \\
\text { Risk }\end{array}$ & $\begin{array}{c}\text { Sortino } \\
\text { Ratio }\end{array}$ & $\begin{array}{c}\text { Risk Europe + } \\
\text { Africa }\end{array}$ & $\begin{array}{c}\text { Return Europe+ } \\
\text { Africa }\end{array}$ & $\begin{array}{c}\text { Sharpe } \\
\text { Ratio }\end{array}$ & $\begin{array}{l}\text { Downside } \\
\text { Risk FHS }\end{array}$ & $\begin{array}{c}\text { Sortino } \\
\text { Ratio }\end{array}$ \\
\hline MVP & $1.464 \%$ & $0.095 \%$ & $1.880 \%$ & $0.908 \%$ & $3.032 \%$ & $0.429 \%$ & $0.143 \%$ & $17.711 \%$ & $0.175 \%$ & $43.369 \%$ \\
\hline $\mathbf{P 2}$ & $1.464 \%$ & $0.097 \%$ & $2.012 \%$ & $0.908 \%$ & $3.243 \%$ & $0.431 \%$ & $0.149 \%$ & $18.934 \%$ & $0.170 \%$ & $48.023 \%$ \\
\hline $\mathbf{P 3}$ & $1.464 \%$ & $0.099 \%$ & $2.143 \%$ & $0.908 \%$ & $3.457 \%$ & $0.445 \%$ & $0.155 \%$ & $19.579 \%$ & $0.178 \%$ & $48.897 \%$ \\
\hline P4 & $1.464 \%$ & $0.101 \%$ & $2.274 \%$ & $0.908 \%$ & $3.669 \%$ & $0.468 \%$ & $0.160 \%$ & $19.816 \%$ & $0.194 \%$ & $47.719 \%$ \\
\hline P5 & $1.465 \%$ & $0.103 \%$ & $2.405 \%$ & $0.908 \%$ & $3.881 \%$ & $0.494 \%$ & $0.166 \%$ & $19.903 \%$ & $0.212 \%$ & $46.380 \%$ \\
\hline P6 & $1.466 \%$ & $0.105 \%$ & $2.535 \%$ & $0.908 \%$ & $4.091 \%$ & $0.523 \%$ & $0.171 \%$ & $19.892 \%$ & $0.230 \%$ & $45.124 \%$ \\
\hline P7 & $1.467 \%$ & $0.107 \%$ & $2.665 \%$ & $0.909 \%$ & $4.300 \%$ & $0.552 \%$ & $0.177 \%$ & $19.850 \%$ & $0.251 \%$ & $43.686 \%$ \\
\hline P8 & $1.468 \%$ & $0.109 \%$ & $2.795 \%$ & $0.909 \%$ & $4.511 \%$ & $0.583 \%$ & $0.183 \%$ & $19.779 \%$ & $0.272 \%$ & $42.401 \%$ \\
\hline P9 & $1.469 \%$ & $0.110 \%$ & $2.924 \%$ & $0.910 \%$ & $4.721 \%$ & $0.614 \%$ & $0.188 \%$ & $19.689 \%$ & $0.294 \%$ & $41.129 \%$ \\
\hline P10 & $1.470 \%$ & $0.112 \%$ & $3.053 \%$ & $0.911 \%$ & $4.927 \%$ & $0.646 \%$ & $0.194 \%$ & $19.584 \%$ & $0.314 \%$ & $40.221 \%$ \\
\hline P11 & $1.472 \%$ & $0.114 \%$ & $3.181 \%$ & $0.912 \%$ & $5.132 \%$ & $0.678 \%$ & $0.200 \%$ & $19.464 \%$ & $0.335 \%$ & $39.440 \%$ \\
\hline P12 & $1.473 \%$ & $0.116 \%$ & $3.309 \%$ & $0.913 \%$ & $5.338 \%$ & $0.712 \%$ & $0.205 \%$ & $19.345 \%$ & $0.355 \%$ & $38.734 \%$ \\
\hline P13 & $1.475 \%$ & $0.118 \%$ & $3.436 \%$ & $0.914 \%$ & $5.546 \%$ & $0.745 \%$ & $0.211 \%$ & $19.237 \%$ & $0.376 \%$ & $38.091 \%$ \\
\hline P14 & $1.476 \%$ & $0.120 \%$ & $3.563 \%$ & $0.914 \%$ & $5.751 \%$ & $0.778 \%$ & $0.216 \%$ & $19.133 \%$ & $0.398 \%$ & $37.431 \%$ \\
\hline P15 & $1.478 \%$ & $0.122 \%$ & $3.689 \%$ & $0.916 \%$ & $5.953 \%$ & $0.812 \%$ & $0.222 \%$ & $19.035 \%$ & $0.419 \%$ & $36.845 \%$ \\
\hline P16 & $1.480 \%$ & $0.124 \%$ & $3.814 \%$ & $0.917 \%$ & $6.154 \%$ & $0.845 \%$ & $0.228 \%$ & $18.942 \%$ & $0.442 \%$ & $36.205 \%$ \\
\hline P17 & $1.482 \%$ & $0.126 \%$ & $3.939 \%$ & $0.919 \%$ & $6.353 \%$ & $0.879 \%$ & $0.233 \%$ & $18.855 \%$ & $0.464 \%$ & $35.743 \%$ \\
\hline P18 & $1.485 \%$ & $0.128 \%$ & $4.062 \%$ & $0.920 \%$ & $6.556 \%$ & $0.913 \%$ & $0.239 \%$ & $18.772 \%$ & $0.485 \%$ & $35.357 \%$ \\
\hline P19 & $1.488 \%$ & $0.130 \%$ & $4.183 \%$ & $0.922 \%$ & $6.750 \%$ & $0.947 \%$ & $0.244 \%$ & $18.693 \%$ & $0.506 \%$ & $34.976 \%$ \\
\hline $\mathbf{P 2 0}$ & $1.491 \%$ & $0.132 \%$ & $4.302 \%$ & $0.924 \%$ & $6.945 \%$ & $0.981 \%$ & $0.250 \%$ & $18.617 \%$ & $0.527 \%$ & $34.658 \%$ \\
\hline P21 & $1.495 \%$ & $0.134 \%$ & $4.420 \%$ & $0.927 \%$ & $7.133 \%$ & $1.015 \%$ & $0.256 \%$ & $18.546 \%$ & $0.549 \%$ & $34.289 \%$ \\
\hline P22 & $1.500 \%$ & $0.136 \%$ & $4.535 \%$ & $0.929 \%$ & $7.322 \%$ & $1.049 \%$ & $0.261 \%$ & $18.478 \%$ & $0.570 \%$ & $33.980 \%$ \\
\hline $\mathbf{P 2 3}$ & $1.505 \%$ & $0.137 \%$ & $4.647 \%$ & $0.932 \%$ & $7.508 \%$ & $1.083 \%$ & $0.267 \%$ & $18.413 \%$ & $0.591 \%$ & $33.722 \%$ \\
\hline P24 & $1.511 \%$ & $0.139 \%$ & $4.756 \%$ & $0.936 \%$ & $7.681 \%$ & $1.117 \%$ & $0.272 \%$ & $18.350 \%$ & $0.613 \%$ & $33.462 \%$ \\
\hline P25 & $1.518 \%$ & $0.141 \%$ & $4.863 \%$ & $0.940 \%$ & $7.851 \%$ & $1.151 \%$ & $0.278 \%$ & $18.291 \%$ & $0.634 \%$ & $33.222 \%$ \\
\hline P26 & $1.525 \%$ & $0.143 \%$ & $4.967 \%$ & $0.944 \%$ & $8.019 \%$ & $1.186 \%$ & $0.284 \%$ & $18.235 \%$ & $0.655 \%$ & $32.998 \%$ \\
\hline P27 & $1.533 \%$ & $0.145 \%$ & $5.067 \%$ & $0.949 \%$ & $8.180 \%$ & $1.221 \%$ & $0.289 \%$ & $18.175 \%$ & $0.675 \%$ & $32.884 \%$ \\
\hline P28 & $1.541 \%$ & $0.147 \%$ & $5.166 \%$ & $0.955 \%$ & $8.337 \%$ & $1.256 \%$ & $0.295 \%$ & $18.114 \%$ & $0.695 \%$ & $32.717 \%$ \\
\hline P29 & $1.550 \%$ & $0.149 \%$ & $5.260 \%$ & $0.960 \%$ & $8.493 \%$ & $1.291 \%$ & $0.301 \%$ & $18.049 \%$ & $0.715 \%$ & $32.594 \%$ \\
\hline P30 & $1.559 \%$ & $0.151 \%$ & $5.352 \%$ & $0.966 \%$ & $8.643 \%$ & $1.327 \%$ & $0.306 \%$ & $17.984 \%$ & $0.737 \%$ & $32.405 \%$ \\
\hline P31 & $1.569 \%$ & $0.153 \%$ & $5.441 \%$ & $0.971 \%$ & $8.790 \%$ & $1.363 \%$ & $0.312 \%$ & $17.919 \%$ & $0.756 \%$ & $32.306 \%$ \\
\hline P32 & $1.580 \%$ & $0.155 \%$ & $5.527 \%$ & $0.977 \%$ & $8.932 \%$ & $1.400 \%$ & $0.317 \%$ & $17.853 \%$ & $0.775 \%$ & $32.231 \%$ \\
\hline P33 & $1.590 \%$ & $0.157 \%$ & $5.611 \%$ & $0.984 \%$ & $9.071 \%$ & $1.436 \%$ & $0.323 \%$ & $17.787 \%$ & $0.797 \%$ & $32.066 \%$ \\
\hline P34 & $1.602 \%$ & $0.159 \%$ & $5.692 \%$ & $0.990 \%$ & $9.206 \%$ & $1.473 \%$ & $0.329 \%$ & $17.724 \%$ & $0.818 \%$ & $31.937 \%$ \\
\hline P35 & $1.613 \%$ & $0.161 \%$ & $5.770 \%$ & $0.997 \%$ & $9.336 \%$ & $1.510 \%$ & $0.334 \%$ & $17.660 \%$ & $0.838 \%$ & $31.839 \%$ \\
\hline P36 & $1.625 \%$ & $0.163 \%$ & $5.847 \%$ & $1.004 \%$ & $9.462 \%$ & $1.548 \%$ & $0.340 \%$ & $17.598 \%$ & $0.859 \%$ & $31.698 \%$ \\
\hline P37 & $1.638 \%$ & $0.164 \%$ & $5.920 \%$ & $1.012 \%$ & $9.584 \%$ & $1.585 \%$ & $0.345 \%$ & $17.538 \%$ & $0.881 \%$ & $31.558 \%$ \\
\hline P38 & $1.650 \%$ & $0.166 \%$ & $5.991 \%$ & $1.020 \%$ & $9.698 \%$ & $1.622 \%$ & $0.351 \%$ & $17.480 \%$ & $0.902 \%$ & $31.443 \%$ \\
\hline P39 & $1.663 \%$ & $0.168 \%$ & $6.061 \%$ & $1.028 \%$ & $9.806 \%$ & $1.660 \%$ & $0.357 \%$ & $17.422 \%$ & $0.922 \%$ & $31.355 \%$ \\
\hline P40 & $1.680 \%$ & $0.170 \%$ & $6.117 \%$ & $1.036 \%$ & $9.918 \%$ & $1.698 \%$ & $0.362 \%$ & $17.363 \%$ & $0.942 \%$ & $31.281 \%$ \\
\hline P41 & $1.701 \%$ & $0.172 \%$ & $6.152 \%$ & $1.047 \%$ & $9.992 \%$ & $1.736 \%$ & $0.368 \%$ & $17.304 \%$ & $0.963 \%$ & $31.190 \%$ \\
\hline P42 & $1.729 \%$ & $0.174 \%$ & $6.166 \%$ & $1.059 \%$ & $10.065 \%$ & $1.775 \%$ & $0.374 \%$ & $17.242 \%$ & $0.985 \%$ & $31.073 \%$ \\
\hline $\mathbf{P 4 3}$ & $1.761 \%$ & $0.176 \%$ & $6.163 \%$ & $1.071 \%$ & $10.132 \%$ & $1.814 \%$ & $0.379 \%$ & $17.175 \%$ & $1.006 \%$ & $30.966 \%$ \\
\hline P44 & $1.796 \%$ & $0.178 \%$ & $6.151 \%$ & $1.085 \%$ & $10.181 \%$ & $1.854 \%$ & $0.385 \%$ & $17.106 \%$ & $1.027 \%$ & $30.877 \%$ \\
\hline P45 & $1.832 \%$ & $0.180 \%$ & $6.135 \%$ & $1.102 \%$ & $10.195 \%$ & $1.895 \%$ & $0.390 \%$ & $17.035 \%$ & $1.050 \%$ & $30.755 \%$ \\
\hline P46 & $1.870 \%$ & $0.182 \%$ & $6.112 \%$ & $1.121 \%$ & $10.200 \%$ & $1.936 \%$ & $0.396 \%$ & $16.964 \%$ & $1.072 \%$ & $30.647 \%$ \\
\hline P47 & $1.910 \%$ & $0.184 \%$ & $6.086 \%$ & $1.138 \%$ & $10.212 \%$ & $1.980 \%$ & $0.402 \%$ & $16.876 \%$ & $1.093 \%$ & $30.566 \%$ \\
\hline P48 & $1.952 \%$ & $0.186 \%$ & $6.054 \%$ & $1.160 \%$ & $10.183 \%$ & $2.027 \%$ & $0.407 \%$ & $16.757 \%$ & $1.115 \%$ & $30.478 \%$ \\
\hline P49 & $2.057 \%$ & $0.188 \%$ & $5.839 \%$ & $1.234 \%$ & $9.735 \%$ & $2.081 \%$ & $0.413 \%$ & $16.591 \%$ & $1.139 \%$ & $30.321 \%$ \\
\hline P50 & $2.335 \%$ & $0.190 \%$ & $5.226 \%$ & $1.411 \%$ & $8.648 \%$ & $2.141 \%$ & $0.418 \%$ & $16.391 \%$ & $1.164 \%$ & $30.159 \%$ \\
\hline
\end{tabular}

Source: author. 
Table 9. Efficient portfolios based in the Filtered Historical Simulation (FHS)

\begin{tabular}{|c|c|c|c|c|c|c|c|c|c|c|c|c|}
\hline \multicolumn{13}{|c|}{ Filtered Historical Simulation model } \\
\hline ortfolios & $\begin{array}{c}\text { Risk } \\
\text { Europe HS } \\
\end{array}$ & $\begin{array}{c}\text { Risk Europe } \\
\text { FHS }\end{array}$ & $\begin{array}{l}\text { Return } \\
\text { Europe }\end{array}$ & $\begin{array}{l}\text { Sharpe } \\
\text { Ratio }\end{array}$ & $\begin{array}{l}\text { Downside } \\
\text { Risk FHS } \\
\end{array}$ & $\begin{array}{l}\text { Sortino } \\
\text { Ratio }\end{array}$ & $\begin{array}{l}\text { Risk Europe } \\
+ \text { Africa HS } \\
\end{array}$ & $\begin{array}{l}\text { Risk Europe } \\
+ \text { Africa FHS } \\
\end{array}$ & $\begin{array}{c}\text { Return Europe } \\
+ \text { Africa }\end{array}$ & $\begin{array}{c}\text { Sharpe } \\
\text { Ratio }\end{array}$ & $\begin{array}{l}\text { Downside } \\
\text { Risk FHS } \\
\end{array}$ & $\begin{array}{c}\text { Sortino } \\
\text { Ratio }\end{array}$ \\
\hline MVP & $4.394 \%$ & $5.710 \%$ & $0.076 \%$ & $0.153 \%$ & $4.637 \%$ & $0.188 \%$ & $3.514 \%$ & $5.087 \%$ & $0.090 \%$ & $0.442 \%$ & $3.545 \%$ & $0.634 \%$ \\
\hline $\mathbf{P 2}$ & $4.745 \%$ & $6.137 \%$ & $0.081 \%$ & $0.223 \%$ & $4.771 \%$ & $0.287 \%$ & $3.540 \%$ & $5.264 \%$ & $0.092 \%$ & $0.471 \%$ & $3.851 \%$ & $0.644 \%$ \\
\hline $\mathbf{P 3}$ & $4.752 \%$ & $5.986 \%$ & $0.086 \%$ & $0.311 \%$ & $4.786 \%$ & $0.389 \%$ & $3.543 \%$ & $5.196 \%$ & $0.090 \%$ & $0.439 \%$ & $3.920 \%$ & $0.582 \%$ \\
\hline P4 & $4.755 \%$ & $5.991 \%$ & $0.068 \%$ & $0.002 \%$ & $4.815 \%$ & $0.003 \%$ & $3.549 \%$ & $5.229 \%$ & $0.093 \%$ & $0.483 \%$ & $3.948 \%$ & $0.640 \%$ \\
\hline P5 & $4.765 \%$ & $5.872 \%$ & $0.080 \%$ & $0.211 \%$ & $4.836 \%$ & $0.257 \%$ & $3.552 \%$ & $4.806 \%$ & $0.093 \%$ & $0.532 \%$ & $3.966 \%$ & $0.645 \%$ \\
\hline P6 & $4.783 \%$ & $5.973 \%$ & $0.068 \%$ & $0.002 \%$ & $4.840 \%$ & $0.003 \%$ & $3.561 \%$ & $250 \%$ & $087 \%$ & $0.363 \%$ & $3.972 \%$ & $0.480 \%$ \\
\hline P7 & $4.799 \%$ & $5.887 \%$ & $0.080 \%$ & $0.205 \%$ & $4.846 \%$ & $0.249 \%$ & $3.562 \%$ & $5.333 \%$ & $0.085 \%$ & $0.332 \%$ & $3.984 \%$ & $0.444 \%$ \\
\hline P8 & $4.818 \%$ & $5.777 \%$ & $0.078 \%$ & $0.182 \%$ & $4.849 \%$ & $0.216 \%$ & $3.564 \%$ & $5.288 \%$ & $0.087 \%$ & $0.362 \%$ & $3.992 \%$ & $0.479 \%$ \\
\hline P9 & $4.844 \%$ & $5.890 \%$ & $0.081 \%$ & $0.229 \%$ & $4.858 \%$ & $0.278 \%$ & $3.570 \%$ & $5.281 \%$ & $086 \%$ & $0.353 \%$ & $3.995 \%$ & $0.467 \%$ \\
\hline P10 & $4.855 \%$ & $5.906 \%$ & $0.080 \%$ & $0.205 \%$ & $4.865 \%$ & $0.249 \%$ & $3.572 \%$ & $5.235 \%$ & $0.087 \%$ & $0.370 \%$ & $3.999 \%$ & $0.484 \%$ \\
\hline P11 & $4.857 \%$ & $5.895 \%$ & $0.068 \%$ & $0.002 \%$ & $4.877 \%$ & $0.003 \%$ & $3.573 \%$ & $5.255 \%$ & $0.086 \%$ & $0.358 \%$ & $4.007 \%$ & $0.470 \%$ \\
\hline P12 & $4.861 \%$ & $5.956 \%$ & $0.081 \%$ & $0.232 \%$ & $4.882 \%$ & $0.283 \%$ & & & $086 \%$ & $0.359 \%$ & $4.021 \%$ & $0.470 \%$ \\
\hline P13 & $4.862 \%$ & $5.810 \%$ & $0.078 \%$ & $0.189 \%$ & $4.883 \%$ & $0.224 \%$ & $3.575 \%$ & $5.235 \%$ & $0.093 \%$ & $0.487 \%$ & $4.057 \%$ & $0.628 \%$ \\
\hline P14 & $4.866 \%$ & $5.780 \%$ & $0.078 \%$ & $0.189 \%$ & $4.884 \%$ & $0.223 \%$ & $3.577 \%$ & $5.293 \%$ & $0.086 \%$ & $0.357 \%$ & $4.058 \%$ & $0.465 \%$ \\
\hline P15 & & & & $0.186 \%$ & & $0.224 \%$ & & & & $0.350 \%$ & $4.058 \%$ & $0.459 \%$ \\
\hline P16 & $4.869 \%$ & $5.823 \%$ & $0.081 \%$ & $0.238 \%$ & $4.888 \%$ & $0.284 \%$ & $3.578 \%$ & $5.283 \%$ & $0.086 \%$ & $0.354 \%$ & $4.061 \%$ & $0.461 \%$ \\
\hline P17 & $4.869 \%$ & $5.884 \%$ & $0.078 \%$ & $0.185 \%$ & $4.888 \%$ & $0.222 \%$ & $3.579 \%$ & $5.276 \%$ & $0.086 \%$ & $0.355 \%$ & $4.064 \%$ & $0.461 \%$ \\
\hline P18 & & & & $0.172 \%$ & & & & & & & & \\
\hline P19 & $4.876 \%$ & $5.822 \%$ & $0.075 \%$ & $0.137 \%$ & $4.893 \%$ & $0.163 \%$ & $3.581 \%$ & $5.314 \%$ & $0.086 \%$ & $0.353 \%$ & $4.066 \%$ & $0.461 \%$ \\
\hline P20 & $4.878 \%$ & $5.836 \%$ & $0.081 \%$ & $0.226 \%$ & $4.893 \%$ & $0.270 \%$ & $3.585 \%$ & $5.363 \%$ & $0.085 \%$ & $0.318 \%$ & $4.067 \%$ & $0.419 \%$ \\
\hline $\mathbf{P 2 1}$ & & & & 0.1 & & & & & & 0 & & $0.479 \%$ \\
\hline P22 & $4.880 \%$ & $5.892 \%$ & $0.082 \%$ & $0.238 \%$ & $4.904 \%$ & $0.287 \%$ & $3.585 \%$ & $5.218 \%$ & $0.086 \%$ & $0.357 \%$ & $4.073 \%$ & $0.457 \%$ \\
\hline $\mathbf{P 2 3}$ & $4.881 \%$ & $5.932 \%$ & $0.081 \%$ & $0.233 \%$ & $4.905 \%$ & $0.282 \%$ & $3.589 \%$ & $5.303 \%$ & $0.086 \%$ & $0.350 \%$ & $4.075 \%$ & $0.455 \%$ \\
\hline P24 & $4.884 \%$ & & $0.079 \%$ & $0.198 \%$ & $4.905 \%$ & $0.239 \%$ & $3.598 \%$ & & $\%$ & $0 \%$ & 4.0 & $0.440 \%$ \\
\hline P25 & $4.885 \%$ & $6.171 \%$ & $0.068 \%$ & $0.002 \%$ & $4.910 \%$ & $0.002 \%$ & $3.603 \%$ & & $0.086 \%$ & $0.357 \%$ & $4.081 \%$ & \\
\hline P26 & $4.889 \%$ & $5.910 \%$ & $0.079 \%$ & $0.188 \%$ & $4.916 \%$ & $0.226 \%$ & $3.611 \%$ & $5.299 \%$ & $0.085 \%$ & $0.335 \%$ & $4.091 \%$ & $0.434 \%$ \\
\hline P27 & $4.889 \%$ & $6.163 \%$ & $0.084 \%$ & $0.265 \%$ & $4.930 \%$ & $0.331 \%$ & $3.614 \%$ & $5.090 \%$ & $0.086 \%$ & $0.368 \%$ & $4.091 \%$ & $0.458 \%$ \\
\hline P28 & & & & & & & & & $0.090 \%$ & $0.428 \%$ & & $0.544 \%$ \\
\hline P29 & $4.896 \%$ & $5.799 \%$ & $0.078 \%$ & $0.176 \%$ & $4.948 \%$ & $0.207 \%$ & $3.624 \%$ & $5.010 \%$ & $0.091 \%$ & $0.467 \%$ & $4.097 \%$ & $0.572 \%$ \\
\hline P30 & $4.900 \%$ & $5.812 \%$ & $0.076 \%$ & $0.147 \%$ & $4.952 \%$ & $0.172 \%$ & $3.650 \%$ & $5.111 \%$ & $0.093 \%$ & $0.495 \%$ & $4.098 \%$ & $0.618 \%$ \\
\hline P31 & & & & $0.136 \%$ & & $0.161 \%$ & & & & & & $0.216 \%$ \\
\hline P32 & $4.908 \%$ & $5.905 \%$ & $0.075 \%$ & $0.132 \%$ & $4.973 \%$ & $0.156 \%$ & $3.676 \%$ & $5.083 \%$ & $0.077 \%$ & $0.196 \%$ & $4.101 \%$ & $0.243 \%$ \\
\hline P33 & $4.912 \%$ & $5.902 \%$ & $0.075 \%$ & $0.134 \%$ & $4.977 \%$ & $0.159 \%$ & $3.686 \%$ & $4.251 \%$ & $0.073 \%$ & $0.118 \%$ & $4.101 \%$ & $0.123 \%$ \\
\hline P34 & $4.919 \%$ & $5.933 \%$ & $0.076 \%$ & $0.136 \%$ & $4.982 \%$ & $0.163 \%$ & $3.700 \%$ & & $0.078 \%$ & $0.203 \%$ & $4.103 \%$ & $0.256 \%$ \\
\hline P35 & $4.920 \%$ & $5.873 \%$ & $0.075 \%$ & $0.136 \%$ & $4.982 \%$ & $0.160 \%$ & $3.710 \%$ & & $0.070 \%$ & $0.056 \%$ & $4.105 \%$ & $0.068 \%$ \\
\hline P36 & $4.924 \%$ & $5.950 \%$ & $0.075 \%$ & $0.123 \%$ & $4.998 \%$ & $0.146 \%$ & $3.713 \%$ & $5.063 \%$ & $0.092 \%$ & $0.475 \%$ & $4.117 \%$ & $0.584 \%$ \\
\hline P37 & $4.928 \%$ & $5.869 \%$ & $0.075 \%$ & $0.128 \%$ & $5.007 \%$ & $0.149 \%$ & $3.716 \%$ & & $0.078 \%$ & $0.216 \%$ & $4.122 \%$ & $0.263 \%$ \\
\hline P38 & $4.940 \%$ & $6.031 \%$ & $0.073 \%$ & $0.096 \%$ & $5.015 \%$ & $0.115 \%$ & $3.729 \%$ & $4.947 \%$ & $0.091 \%$ & $0.471 \%$ & $4.123 \%$ & $0.565 \%$ \\
\hline P39 & $5.010 \%$ & $5.890 \%$ & $0.068 \%$ & $0.002 \%$ & $5.019 \%$ & $0.003 \%$ & $3.771 \%$ & $4.979 \%$ & $0.068 \%$ & $0.006 \%$ & $4.129 \%$ & $0.007 \%$ \\
\hline P40 & & $6.066 \%$ & $0.068 \%$ & $0.003 \%$ & $5.028 \%$ & $0.003 \%$ & $3.779 \%$ & & $0.069 \%$ & $0.028 \%$ & $4.134 \%$ & $0.034 \%$ \\
\hline P41 & $5.034 \%$ & $5.905 \%$ & $0.068 \%$ & $0.002 \%$ & $5.037 \%$ & $0.002 \%$ & $3.800 \%$ & $4.985 \%$ & $0.071 \%$ & $0.080 \%$ & $4.136 \%$ & $0.096 \%$ \\
\hline P42 & $5.035 \%$ & $5.960 \%$ & $0.073 \%$ & $0.091 \%$ & $5.048 \%$ & $0.108 \%$ & $3.801 \%$ & $5.059 \%$ & $0.069 \%$ & $0.031 \%$ & $4.141 \%$ & $0.038 \%$ \\
\hline $\mathbf{P 4 3}$ & $5.059 \%$ & $6.014 \%$ & $0.071 \%$ & $0.051 \%$ & $5.055 \%$ & $0.061 \%$ & $3.805 \%$ & $5.129 \%$ & $0.074 \%$ & $0.121 \%$ & $4.143 \%$ & $0.150 \%$ \\
\hline P44 & $5.062 \%$ & $5.917 \%$ & $0.068 \%$ & $0.001 \%$ & $5.061 \%$ & $0.001 \%$ & $3.820 \%$ & $5.002 \%$ & $0.071 \%$ & $0.063 \%$ & $4.144 \%$ & $0.076 \%$ \\
\hline P45 & $5.082 \%$ & $6.113 \%$ & $0.071 \%$ & $0.062 \%$ & $5.080 \%$ & $0.074 \%$ & $3.836 \%$ & $5.036 \%$ & $0.068 \%$ & $0.018 \%$ & $4.153 \%$ & $0.022 \%$ \\
\hline P46 & $5.108 \%$ & $5.853 \%$ & $0.071 \%$ & $0.051 \%$ & $5.093 \%$ & $0.059 \%$ & $3.851 \%$ & $4.863 \%$ & $0.069 \%$ & $0.041 \%$ & $4.177 \%$ & $0.047 \%$ \\
\hline P47 & $5.114 \%$ & $6.256 \%$ & $0.072 \%$ & $0.077 \%$ & $5.094 \%$ & $0.095 \%$ & $3.864 \%$ & $5.009 \%$ & $0.069 \%$ & $0.040 \%$ & $4.180 \%$ & $0.048 \%$ \\
\hline P48 & $5.127 \%$ & $6.062 \%$ & $0.071 \%$ & $0.059 \%$ & $5.101 \%$ & $0.070 \%$ & $3.876 \%$ & $5.042 \%$ & $0.070 \%$ & $0.051 \%$ & $4.181 \%$ & $0.062 \%$ \\
\hline P49 & $5.139 \%$ & $5.982 \%$ & $0.069 \%$ & $0.024 \%$ & $5.141 \%$ & $0.028 \%$ & $3.914 \%$ & $5.017 \%$ & $0.071 \%$ & $0.064 \%$ & $4.184 \%$ & $0.077 \%$ \\
\hline P50 & $5.148 \%$ & $6.051 \%$ & $0.073 \%$ & $0.090 \%$ & $5.156 \%$ & $0.105 \%$ & $3.914 \%$ & $5.096 \%$ & $0.072 \%$ & $0.079 \%$ & $4.215 \%$ & $0.095 \%$ \\
\hline
\end{tabular}

Source: author. 


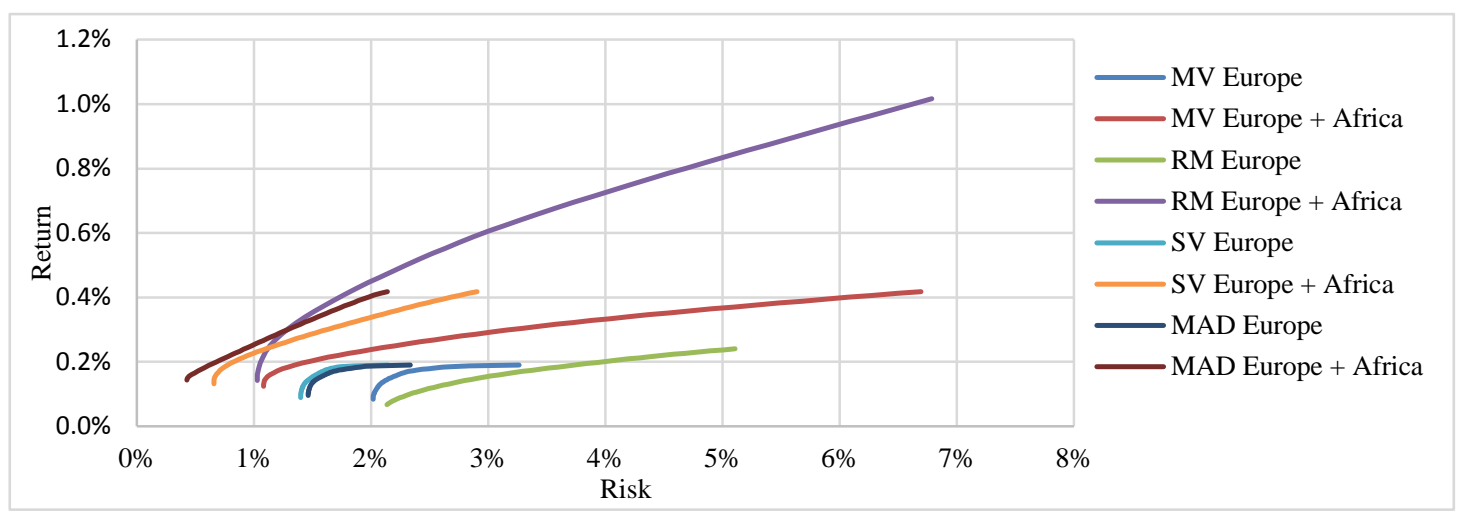

Figure 1. Efficient frontiers of the investment strategies

This figure shows us the efficient frontiers of the investment strategies for each optimization model start from period August 05, 2004 to July 07, 2016 based in the criteria risk and return. As we can to see the diversification strategy of European investment portfolio with African assets shown to be more efficient than European investment portfolios, for all optimization models. However, there are some diversified European investment portfolios with African assets show riskier than European investment portfolios but show better return.

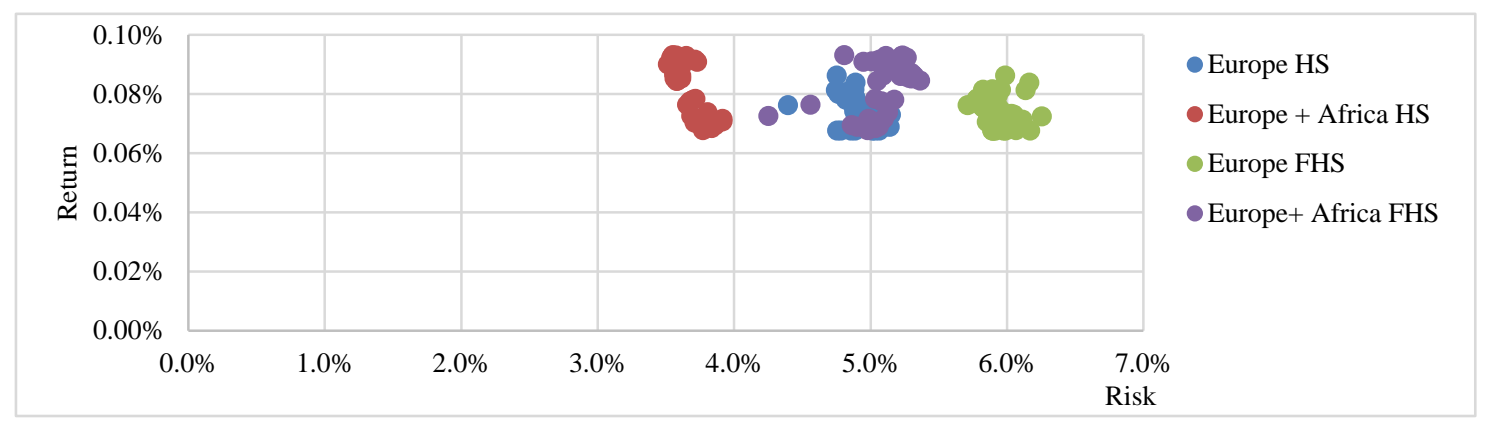

Figure 2. Efficient frontiers of the investment strategies by FHS method

This figure shows us the efficient frontiers of the investment strategies by FHS optimization model start from period August 05, 2004 to July 07, 2016 based in the criteria risk and return. This our contribution for literature, then we think the efficient portfolios representations by point is better than line because the convex properties of model. We remember that this model combine Historical Simulation (HS) method with GARCH volatility model. As shows the figure, for both HS and FHS methods the diversification strategy of European investment portfolio with African assets shown to be more efficient than European investment portfolios, that is, show lesser risk and more return.

Table 10. The average performance of the investment strategies

\begin{tabular}{|c|c|c|c|c|c|c|c|c|c|c|}
\hline \multirow[t]{2}{*}{ Models/Indicators } & \multicolumn{2}{|c|}{ Mean Variance } & \multicolumn{2}{|c|}{ Resample Michaud } & \multicolumn{2}{|c|}{ SemiVariance } & \multicolumn{2}{|c|}{ Mean Absolute Devition } & \multicolumn{2}{|c|}{$\begin{array}{c}\text { Filtered Historical } \\
\text { Simulation }\end{array}$} \\
\hline & Europe & $\begin{array}{c}\text { Europe + } \\
\text { Africa } \\
\end{array}$ & Europe & $\begin{array}{c}\text { Europe + } \\
\text { Africa } \\
\end{array}$ & Europe & $\begin{array}{l}\text { Europe } \\
+ \text { Africa }\end{array}$ & Europe & $\begin{array}{c}\text { Europe }+ \\
\text { Africa } \\
\end{array}$ & Europe & $\begin{array}{c}\text { Europe + } \\
\text { Africa } \\
\end{array}$ \\
\hline Risk & $2.197 \%$ & $3.025 \%$ & $3.223 \%$ & $3.199 \%$ & $1.504 \%$ & $1.513 \%$ & $1.597 \%$ & $1.194 \%$ & $5.930 \%$ & $5.118 \%$ \\
\hline Return & $0.136 \%$ & $0.271 \%$ & $0.154 \%$ & $0.579 \%$ & $0.139 \%$ & $0.275 \%$ & $0.142 \%$ & $0.281 \%$ & $0.075 \%$ & $0.082 \%$ \\
\hline Sharpe Ratio & $3.054 \%$ & $7.273 \%$ & $2.451 \%$ & $16.292 \%$ & $4.666 \%$ & $14.026 \%$ & $4.585 \%$ & $18.265 \%$ & $0.134 \%$ & $0.284 \%$ \\
\hline Downside Risk & $1.508 \%$ & $1.537 \%$ & $1.825 \%$ & $1.676 \%$ & $1.504 \%$ & $1.513 \%$ & $0.983 \%$ & $0.643 \%$ & $4.940 \%$ & $4.067 \%$ \\
\hline Sortino Ratio & $4.463 \%$ & $13.310 \%$ & $4.329 \%$ & $30.976 \%$ & $4.666 \%$ & $14.026 \%$ & $7.456 \%$ & $35.429 \%$ & $0.161 \%$ & $0.364 \%$ \\
\hline
\end{tabular}

This table illustrates the average contribution in terms risk and return of the investment strategies and their performances through Sharpe Ratio and Sortino Ratio. To MV and SV models, the Diversification of European pinvestment portfolios with African assets is riskier than European investment portfolio but presents better return and performance. To RM, MAD and FHS models, the Diversification of European investment portfolios with 
African assets show to be more efficient than European investment portfolio

Table 11. Statistical test results of the performance of the investment strategies

\begin{tabular}{ccccc}
\hline Models & & \multicolumn{2}{c}{ Test result Sharpe Ratio (SR) } & Reject or No reject \\
\hline Mean Variance (MV) & Null hypotheses & t-statistic & P-value & No Reject \\
Resample Michaud (RM) & SR2>SR1 & 17.19 & 0.000 & No Reject \\
SemiVariance (SV) & SR2>SR1 & 34.25 & 0.000 & No Reject \\
Mean Absolute Deviation (MAD) & SR2>SR1 & 29.40 & 0.000 & No Reject \\
Filtered Historical Simulation (FHS) & SR2>SR1 & 57.13 & 0.000 & No Reject \\
\hline & SR2>SR1 & 5.85 & 0.000 & Reject or No reject \\
\hline Models & Null hypotheses & test result Sortino Ratio (S) & P-value & No Reject \\
\hline Mean Variance (MV) & S2>S1 & 27.04 & 0.0000 & No Reject \\
Resample Michaud (RM) & S2>S1 & 34.68 & 0.0000 & No Reject \\
SemiVariance (SV) & S2>S1 & 29.40 & 0.0000 & No Reject
\end{tabular}

The table 11shows us the statistical test result of the performance of investment strategies. where SR2 and S2 corresponding to the strategy 2 performances and SR1 and S1 are strategy 1 performances. Thus, we considered $1 \%$ significance level. We can to see high t-statistic value to all optimization models and p-value less $1 \%$, it that mean not rejection of null hypotheses. Then, we say that the Diversification of European investment portfolios with African assets presents better performance than European investment portfolio without diversification.

Table 12. The average contribution of the Europe portfolio diversification with African capital market assets

\begin{tabular}{lccccc}
\hline Contribuition Measure & MV & RM & SV & MAD & FHS \\
\hline Overall Benefit & $0.176 \%$ & $0.488 \%$ & $0.187 \%$ & $0.198 \%$ & $0.013 \%$ \\
Diversification Benefit & $0.041 \%$ & $0.063 \%$ & $0.051 \%$ & $0.059 \%$ & $0.006 \%$ \\
Return Benefit & $0.135 \%$ & $0.425 \%$ & $0.136 \%$ & $0.139 \%$ & $0.007 \%$ \\
\hline
\end{tabular}

The table 12 represents the real contribution of the Europe portfolio diversification with African capital market assets. based in equation 22, 23 e 24 considering all optimization models used in this study. To all optimization models, the Diversification of European investment portfolios with African assets generates benefits in the returns and diversification that correspond the overall benefits.

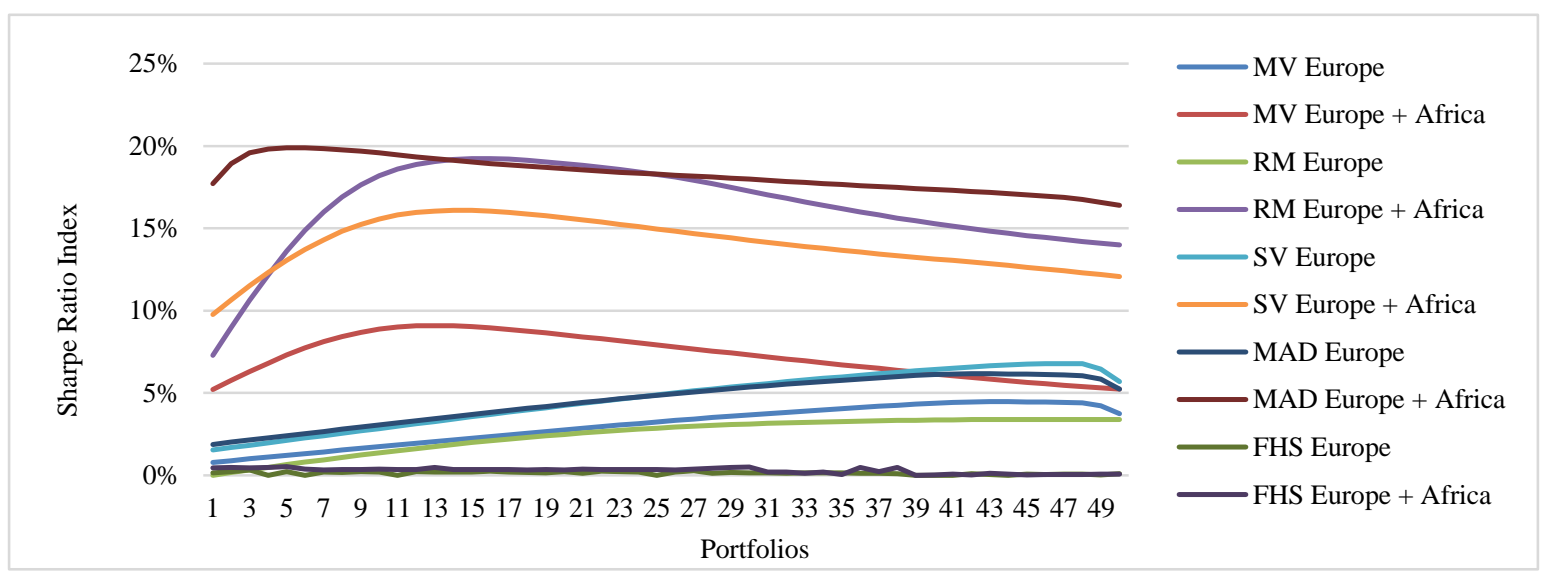

Figure 3. Portfolio performances of the investment strategies for each optimization models by Sharpe Ratio

The figure 3 shows us the portfolio performances of the investment strategies for each optimization model measured by Sharpe Ratio. However, investment strategy with higher value of Sharpe Ratio, show better performance. The diversification strategy of European investment portfolio with African assets shown to have better performances than European investment portfolios, for all optimization models. 


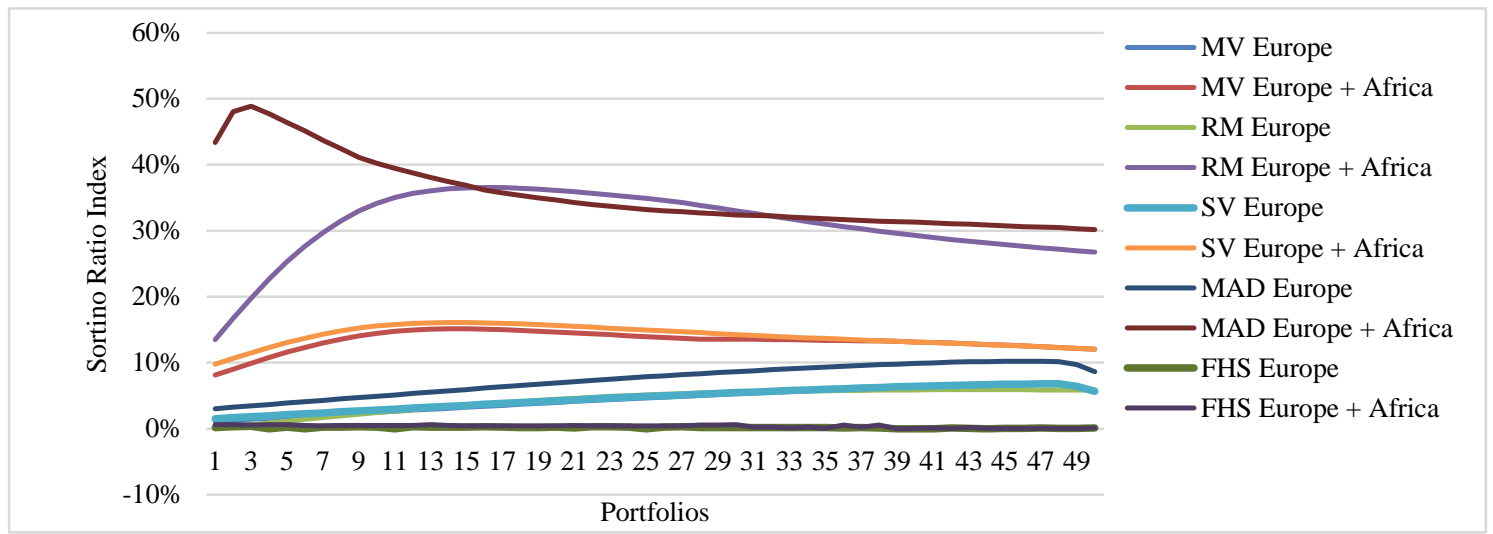

Figure 4. Portfolio performances of the investment strategies for each optimization models by Sortino Ratio

The figure 4 shows us the portfolio performances of the investment strategies for each optimization model measured by Sharpe Ratio. However, investment strategy with higher value of Sortino Ratio, show better performance. The diversification strategy of European investment portfolio with African assets shown to have better performances than European investment portfolios, for all optimization models.

Table 13. Portfolio weights by Mean Variance model

\begin{tabular}{|c|c|c|c|c|c|c|c|c|c|c|c|c|c|c|c|}
\hline Portfolios & Namibi & igeria & Gongo & $\begin{array}{c}\text { Cote } \\
\text { D'Ivoire }\end{array}$ & Egy & Morrocco & Tunisia & Botswana & Mauritius & Kenya & Uganda & Zambia & $\begin{array}{l}\text { South } \\
\text { Africa }\end{array}$ & Europe & $\begin{array}{c}\text { Total } \\
\text { PortfolioWeight }\end{array}$ \\
\hline MPV & $0 \%$ & $1 \%$ & $26 \%$ & $2 \%$ & $0 \%$ & $0 \%$ & $24 \%$ & $15 \%$ & $12 \%$ & $4 \%$ & $1 \%$ & $3 \%$ & $0 \%$ & $9 \%$ & $100 \%$ \\
\hline P2 & $0 \%$ & $1 \%$ & $27 \%$ & $3 \%$ & $0 \%$ & $0 \%$ & $25 \%$ & $15 \%$ & $13 \%$ & $3 \%$ & $1 \%$ & $4 \%$ & $0 \%$ & $9 \%$ & $100 \%$ \\
\hline P3 & $0 \%$ & $1 \%$ & $27 \%$ & $3 \%$ & $0 \%$ & $0 \%$ & $25 \%$ & $14 \%$ & $13 \%$ & $2 \%$ & $2 \%$ & $4 \%$ & $0 \%$ & $10 \%$ & $100 \%$ \\
\hline P4 & $0 \%$ & $0 \%$ & $27 \%$ & $3 \%$ & $0 \%$ & $0 \%$ & $25 \%$ & $13 \%$ & $13 \%$ & $0 \%$ & $3 \%$ & $5 \%$ & $0 \%$ & $10 \%$ & $100 \%$ \\
\hline P5 & $0 \%$ & $0 \%$ & $27 \%$ & $4 \%$ & $0 \%$ & $0 \%$ & $25 \%$ & $11 \%$ & $12 \%$ & $0 \%$ & $3 \%$ & $6 \%$ & $0 \%$ & $11 \%$ & $100 \%$ \\
\hline P6 & $0 \%$ & $0 \%$ & $27 \%$ & $5 \%$ & $0 \%$ & $0 \%$ & $26 \%$ & $8 \%$ & $12 \%$ & $0 \%$ & $3 \%$ & $7 \%$ & $0 \%$ & $12 \%$ & $100 \%$ \\
\hline P7 & $0 \%$ & $0 \%$ & $28 \%$ & $6 \%$ & $0 \%$ & $0 \%$ & $26 \%$ & $6 \%$ & $11 \%$ & $0 \%$ & $3 \%$ & $9 \%$ & $0 \%$ & $12 \%$ & $100 \%$ \\
\hline P8 & $0 \%$ & $0 \%$ & $28 \%$ & $7 \%$ & $0 \%$ & $0 \%$ & $26 \%$ & $3 \%$ & $10 \%$ & $0 \%$ & $3 \%$ & $10 \%$ & $0 \%$ & $13 \%$ & $100 \%$ \\
\hline P9 & $0 \%$ & $0 \%$ & $28 \%$ & $7 \%$ & $0 \%$ & $0 \%$ & $27 \%$ & $0 \%$ & $10 \%$ & $0 \%$ & $3 \%$ & $11 \%$ & $0 \%$ & $14 \%$ & $100 \%$ \\
\hline P10 & $0 \%$ & $0 \%$ & $28 \%$ & $9 \%$ & $0 \%$ & $0 \%$ & $26 \%$ & $0 \%$ & $8 \%$ & $0 \%$ & $3 \%$ & $13 \%$ & $0 \%$ & $14 \%$ & $100 \%$ \\
\hline P11 & $0 \%$ & $0 \%$ & $27 \%$ & $10 \%$ & $0 \%$ & $0 \%$ & $24 \%$ & $0 \%$ & $7 \%$ & $0 \%$ & $3 \%$ & $14 \%$ & $0 \%$ & $15 \%$ & $100 \%$ \\
\hline P12 & $0 \%$ & $0 \%$ & $27 \%$ & $11 \%$ & $0 \%$ & $0 \%$ & $23 \%$ & $0 \%$ & $5 \%$ & $0 \%$ & $2 \%$ & $16 \%$ & $0 \%$ & $15 \%$ & $100 \%$ \\
\hline P13 & $0 \%$ & $0 \%$ & $27 \%$ & $13 \%$ & $0 \%$ & $0 \%$ & $22 \%$ & $0 \%$ & $3 \%$ & $0 \%$ & $2 \%$ & $18 \%$ & $0 \%$ & $15 \%$ & $100 \%$ \\
\hline P14 & $0 \%$ & $0 \%$ & $26 \%$ & $14 \%$ & $1 \%$ & $0 \%$ & $20 \%$ & $0 \%$ & $2 \%$ & $0 \%$ & $2 \%$ & $19 \%$ & $0 \%$ & $16 \%$ & $100 \%$ \\
\hline P15 & $0 \%$ & $0 \%$ & $26 \%$ & $15 \%$ & $1 \%$ & $0 \%$ & $18 \%$ & $0 \%$ & $0 \%$ & $0 \%$ & $2 \%$ & $21 \%$ & $0 \%$ & $17 \%$ & $100 \%$ \\
\hline P16 & $0 \%$ & $0 \%$ & $25 \%$ & $17 \%$ & $0 \%$ & $0 \%$ & $16 \%$ & $0 \%$ & $0 \%$ & $0 \%$ & $1 \%$ & $22 \%$ & $0 \%$ & $18 \%$ & $100 \%$ \\
\hline P17 & $0 \%$ & $0 \%$ & $24 \%$ & $19 \%$ & $0 \%$ & $0 \%$ & $13 \%$ & $0 \%$ & $0 \%$ & $0 \%$ & $1 \%$ & $24 \%$ & $0 \%$ & $19 \%$ & $100 \%$ \\
\hline P18 & $0 \%$ & $0 \%$ & $23 \%$ & $20 \%$ & $0 \%$ & $0 \%$ & $10 \%$ & $0 \%$ & $0 \%$ & $0 \%$ & $0 \%$ & $26 \%$ & $0 \%$ & $21 \%$ & $100 \%$ \\
\hline P19 & $0 \%$ & $0 \%$ & $22 \%$ & $22 \%$ & $0 \%$ & $0 \%$ & $7 \%$ & $0 \%$ & $0 \%$ & $0 \%$ & $0 \%$ & $27 \%$ & $0 \%$ & $22 \%$ & $100 \%$ \\
\hline P20 & $0 \%$ & $0 \%$ & $21 \%$ & $23 \%$ & $0 \%$ & $0 \%$ & $4 \%$ & $0 \%$ & $0 \%$ & $0 \%$ & $0 \%$ & $29 \%$ & $0 \%$ & $23 \%$ & $100 \%$ \\
\hline P21 & $0 \%$ & $0 \%$ & $20 \%$ & $25 \%$ & $0 \%$ & $0 \%$ & $1 \%$ & $0 \%$ & $0 \%$ & $0 \%$ & $0 \%$ & $30 \%$ & $0 \%$ & $24 \%$ & $100 \%$ \\
\hline P22 & $0 \%$ & $0 \%$ & $18 \%$ & $27 \%$ & $0 \%$ & $0 \%$ & $0 \%$ & $0 \%$ & $0 \%$ & $0 \%$ & $0 \%$ & $32 \%$ & $0 \%$ & $24 \%$ & $100 \%$ \\
\hline P23 & $0 \%$ & $0 \%$ & $15 \%$ & $29 \%$ & $0 \%$ & $0 \%$ & $0 \%$ & $0 \%$ & $0 \%$ & $0 \%$ & $0 \%$ & $33 \%$ & $0 \%$ & $23 \%$ & $100 \%$ \\
\hline P24 & $0 \%$ & $0 \%$ & $12 \%$ & $30 \%$ & $0 \%$ & $0 \%$ & $0 \%$ & $0 \%$ & $0 \%$ & $0 \%$ & $0 \%$ & $35 \%$ & $0 \%$ & $23 \%$ & $100 \%$ \\
\hline P25 & $0 \%$ & $0 \%$ & $9 \%$ & $32 \%$ & $0 \%$ & $0 \%$ & $0 \%$ & $0 \%$ & $0 \%$ & $0 \%$ & $0 \%$ & $36 \%$ & $0 \%$ & $23 \%$ & $100 \%$ \\
\hline P26 & $0 \%$ & $0 \%$ & $6 \%$ & $34 \%$ & $0 \%$ & $0 \%$ & $0 \%$ & $0 \%$ & $0 \%$ & $0 \%$ & $0 \%$ & $37 \%$ & $0 \%$ & $22 \%$ & $100 \%$ \\
\hline P27 & $0 \%$ & $0 \%$ & $3 \%$ & $36 \%$ & $0 \%$ & $0 \%$ & $0 \%$ & $0 \%$ & $0 \%$ & $0 \%$ & $0 \%$ & $39 \%$ & $0 \%$ & $22 \%$ & $100 \%$ \\
\hline P28 & $0 \%$ & $0 \%$ & $0 \%$ & $38 \%$ & $0 \%$ & $0 \%$ & $0 \%$ & $0 \%$ & $0 \%$ & $0 \%$ & $0 \%$ & $40 \%$ & $0 \%$ & $22 \%$ & $100 \%$ \\
\hline P29 & $0 \%$ & $0 \%$ & $0 \%$ & $41 \%$ & $0 \%$ & $0 \%$ & $0 \%$ & $0 \%$ & $0 \%$ & $0 \%$ & $0 \%$ & $41 \%$ & $0 \%$ & $18 \%$ & $100 \%$ \\
\hline P30 & $0 \%$ & $0 \%$ & $0 \%$ & $43 \%$ & $0 \%$ & $0 \%$ & $0 \%$ & $0 \%$ & $0 \%$ & $0 \%$ & $0 \%$ & $42 \%$ & $0 \%$ & $15 \%$ & $100 \%$ \\
\hline P31 & $0 \%$ & $0 \%$ & $0 \%$ & $46 \%$ & $0 \%$ & $0 \%$ & $0 \%$ & $0 \%$ & $0 \%$ & $0 \%$ & $0 \%$ & $42 \%$ & $0 \%$ & $12 \%$ & $100 \%$ \\
\hline P32 & $0 \%$ & $0 \%$ & $0 \%$ & $48 \%$ & $0 \%$ & $0 \%$ & $0 \%$ & $0 \%$ & $0 \%$ & $0 \%$ & $0 \%$ & $43 \%$ & $0 \%$ & $9 \%$ & $100 \%$ \\
\hline P33 & $0 \%$ & $0 \%$ & $0 \%$ & $51 \%$ & $0 \%$ & $0 \%$ & $0 \%$ & $0 \%$ & $0 \%$ & $0 \%$ & $0 \%$ & $43 \%$ & $0 \%$ & $6 \%$ & $100 \%$ \\
\hline P34 & $0 \%$ & $0 \%$ & $0 \%$ & $53 \%$ & $0 \%$ & $0 \%$ & $0 \%$ & $0 \%$ & $0 \%$ & $0 \%$ & $0 \%$ & $44 \%$ & $0 \%$ & $3 \%$ & $100 \%$ \\
\hline P35 & $0 \%$ & $0 \%$ & $0 \%$ & $56 \%$ & $0 \%$ & $0 \%$ & $0 \%$ & $0 \%$ & $0 \%$ & $0 \%$ & $0 \%$ & $44 \%$ & $0 \%$ & $0 \%$ & $100 \%$ \\
\hline P36 & $0 \%$ & $0 \%$ & $0 \%$ & $59 \%$ & $0 \%$ & $0 \%$ & $0 \%$ & $0 \%$ & $0 \%$ & $0 \%$ & $0 \%$ & $41 \%$ & $0 \%$ & $0 \%$ & $100 \%$ \\
\hline
\end{tabular}




\begin{tabular}{llllllllllllllll}
\hline P37 & $0 \%$ & $0 \%$ & $0 \%$ & $61 \%$ & $0 \%$ & $0 \%$ & $0 \%$ & $0 \%$ & $0 \%$ & $0 \%$ & $0 \%$ & $39 \%$ & $0 \%$ & $\mathbf{0 \%}$ & $100 \%$ \\
P38 & $0 \%$ & $0 \%$ & $0 \%$ & $64 \%$ & $0 \%$ & $0 \%$ & $0 \%$ & $0 \%$ & $0 \%$ & $0 \%$ & $0 \%$ & $36 \%$ & $0 \%$ & $\mathbf{0 \%}$ & $100 \%$ \\
$\mathbf{P 3 9}$ & $0 \%$ & $0 \%$ & $0 \%$ & $67 \%$ & $0 \%$ & $0 \%$ & $0 \%$ & $0 \%$ & $0 \%$ & $0 \%$ & $0 \%$ & $33 \%$ & $0 \%$ & $\mathbf{0 \%}$ & $100 \%$ \\
$\mathbf{P 4 0}$ & $0 \%$ & $0 \%$ & $0 \%$ & $70 \%$ & $0 \%$ & $0 \%$ & $0 \%$ & $0 \%$ & $0 \%$ & $0 \%$ & $0 \%$ & $30 \%$ & $0 \%$ & $\mathbf{0 \%}$ & $100 \%$ \\
$\mathbf{P 4 1}$ & $0 \%$ & $0 \%$ & $0 \%$ & $73 \%$ & $0 \%$ & $0 \%$ & $0 \%$ & $0 \%$ & $0 \%$ & $0 \%$ & $0 \%$ & $27 \%$ & $0 \%$ & $\mathbf{0 \%}$ & $100 \%$ \\
$\mathbf{P 4 2}$ & $0 \%$ & $0 \%$ & $0 \%$ & $76 \%$ & $0 \%$ & $0 \%$ & $0 \%$ & $0 \%$ & $0 \%$ & $0 \%$ & $0 \%$ & $24 \%$ & $0 \%$ & $\mathbf{0 \%}$ & $100 \%$ \\
$\mathbf{P 4 3}$ & $0 \%$ & $0 \%$ & $0 \%$ & $79 \%$ & $0 \%$ & $0 \%$ & $0 \%$ & $0 \%$ & $0 \%$ & $0 \%$ & $0 \%$ & $21 \%$ & $0 \%$ & $\mathbf{0 \%}$ & $100 \%$ \\
$\mathbf{P 4 4}$ & $0 \%$ & $0 \%$ & $0 \%$ & $82 \%$ & $0 \%$ & $0 \%$ & $0 \%$ & $0 \%$ & $0 \%$ & $0 \%$ & $0 \%$ & $18 \%$ & $0 \%$ & $\mathbf{0 \%}$ & $100 \%$ \\
$\mathbf{P 4 5}$ & $0 \%$ & $0 \%$ & $0 \%$ & $85 \%$ & $0 \%$ & $0 \%$ & $0 \%$ & $0 \%$ & $0 \%$ & $0 \%$ & $0 \%$ & $15 \%$ & $0 \%$ & $\mathbf{0 \%}$ & $100 \%$ \\
$\mathbf{P 4 6}$ & $0 \%$ & $0 \%$ & $0 \%$ & $88 \%$ & $0 \%$ & $0 \%$ & $0 \%$ & $0 \%$ & $0 \%$ & $0 \%$ & $0 \%$ & $12 \%$ & $0 \%$ & $\mathbf{0 \%}$ & $100 \%$ \\
$\mathbf{P 4 7}$ & $0 \%$ & $0 \%$ & $0 \%$ & $91 \%$ & $0 \%$ & $0 \%$ & $0 \%$ & $0 \%$ & $0 \%$ & $0 \%$ & $0 \%$ & $9 \%$ & $0 \%$ & $\mathbf{0 \%}$ & $100 \%$ \\
$\mathbf{P 4 8}$ & $0 \%$ & $0 \%$ & $0 \%$ & $94 \%$ & $0 \%$ & $0 \%$ & $0 \%$ & $0 \%$ & $0 \%$ & $0 \%$ & $0 \%$ & $6 \%$ & $0 \%$ & $\mathbf{0 \%}$ & $100 \%$ \\
$\mathbf{P 4 9}$ & $0 \%$ & $0 \%$ & $0 \%$ & $97 \%$ & $0 \%$ & $0 \%$ & $0 \%$ & $0 \%$ & $0 \%$ & $0 \%$ & $0 \%$ & $3 \%$ & $0 \%$ & $\mathbf{0 \%}$ & $100 \%$ \\
P50 & $0 \%$ & $0 \%$ & $0 \%$ & $100 \%$ & $0 \%$ & $0 \%$ & $0 \%$ & $0 \%$ & $0 \%$ & $0 \%$ & $0 \%$ & $0 \%$ & $0 \%$ & $\mathbf{0 \%}$ & $100 \%$ \\
\hline
\end{tabular}

Table 14. Portfolio weights by Resample Michaud model

\begin{tabular}{|c|c|c|c|c|c|c|c|c|c|c|c|c|c|c|c|}
\hline Portfolios & Namibi & Nigeria & Gongo & $\begin{array}{c}\text { Cote } \\
\text { D'Ivoire }\end{array}$ & Egypt & t Morrocco & Tunisia & Botswana & Mauritius & s Kenya & Uganda & Zambia & $\begin{array}{l}\text { South } \\
\text { Africa }\end{array}$ & Europe & $\begin{array}{c}\text { Total } \\
\text { PortfolioWeight }\end{array}$ \\
\hline MPV & $0 \%$ & $2 \%$ & $27 \%$ & $1 \%$ & $0 \%$ & $0 \%$ & $26 \%$ & $16 \%$ & $13 \%$ & $3 \%$ & $0 \%$ & $2 \%$ & $0 \%$ & $9 \%$ & $100 \%$ \\
\hline P2 & $0 \%$ & $2 \%$ & $27 \%$ & $2 \%$ & $0 \%$ & $1 \%$ & $25 \%$ & $17 \%$ & $15 \%$ & $3 \%$ & $0 \%$ & $2 \%$ & $0 \%$ & $7 \%$ & $100 \%$ \\
\hline P3 & $0 \%$ & $1 \%$ & $28 \%$ & $2 \%$ & $0 \%$ & $1 \%$ & $24 \%$ & $17 \%$ & $17 \%$ & $2 \%$ & $0 \%$ & $1 \%$ & $0 \%$ & $5 \%$ & $100 \%$ \\
\hline P4 & $0 \%$ & $1 \%$ & $28 \%$ & $3 \%$ & $0 \%$ & $1 \%$ & $23 \%$ & $18 \%$ & $20 \%$ & $1 \%$ & $0 \%$ & $0 \%$ & $0 \%$ & $4 \%$ & $100 \%$ \\
\hline P5 & $0 \%$ & $1 \%$ & $28 \%$ & $4 \%$ & $0 \%$ & $2 \%$ & $22 \%$ & $18 \%$ & $22 \%$ & $0 \%$ & $0 \%$ & $0 \%$ & $0 \%$ & $4 \%$ & $100 \%$ \\
\hline P6 & $0 \%$ & $0 \%$ & $28 \%$ & $5 \%$ & $0 \%$ & $1 \%$ & $21 \%$ & $18 \%$ & $25 \%$ & $0 \%$ & $0 \%$ & $0 \%$ & $0 \%$ & $2 \%$ & $100 \%$ \\
\hline P7 & $0 \%$ & $0 \%$ & $27 \%$ & $6 \%$ & $0 \%$ & $1 \%$ & $19 \%$ & $18 \%$ & $27 \%$ & $0 \%$ & $0 \%$ & $0 \%$ & $0 \%$ & $1 \%$ & $100 \%$ \\
\hline P8 & $0 \%$ & $0 \%$ & $27 \%$ & $7 \%$ & $0 \%$ & $1 \%$ & $17 \%$ & $18 \%$ & $30 \%$ & $0 \%$ & $0 \%$ & $0 \%$ & $0 \%$ & $0 \%$ & $100 \%$ \\
\hline P9 & $0 \%$ & $0 \%$ & $26 \%$ & $9 \%$ & $0 \%$ & $1 \%$ & $15 \%$ & $17 \%$ & $32 \%$ & $0 \%$ & $0 \%$ & $0 \%$ & $0 \%$ & $0 \%$ & $100 \%$ \\
\hline P10 & $0 \%$ & $0 \%$ & $25 \%$ & $10 \%$ & $0 \%$ & $1 \%$ & $12 \%$ & $16 \%$ & $35 \%$ & $0 \%$ & $0 \%$ & $0 \%$ & $0 \%$ & $0 \%$ & $100 \%$ \\
\hline P11 & $0 \%$ & $0 \%$ & $25 \%$ & $12 \%$ & $0 \%$ & $1 \%$ & $9 \%$ & $16 \%$ & $37 \%$ & $0 \%$ & $0 \%$ & $0 \%$ & $0 \%$ & $0 \%$ & $100 \%$ \\
\hline P12 & $0 \%$ & $0 \%$ & $24 \%$ & $13 \%$ & $0 \%$ & $1 \%$ & $7 \%$ & $15 \%$ & $40 \%$ & $0 \%$ & $0 \%$ & $0 \%$ & $0 \%$ & $0 \%$ & $100 \%$ \\
\hline P13 & $0 \%$ & $0 \%$ & $23 \%$ & $14 \%$ & $0 \%$ & $2 \%$ & $4 \%$ & $14 \%$ & $43 \%$ & $0 \%$ & $0 \%$ & $0 \%$ & $0 \%$ & $0 \%$ & $100 \%$ \\
\hline P14 & $0 \%$ & $0 \%$ & $22 \%$ & $16 \%$ & $0 \%$ & $2 \%$ & $1 \%$ & $14 \%$ & $45 \%$ & $0 \%$ & $0 \%$ & $0 \%$ & $0 \%$ & $0 \%$ & $100 \%$ \\
\hline P15 & $0 \%$ & $0 \%$ & $21 \%$ & $17 \%$ & $0 \%$ & $1 \%$ & $0 \%$ & $12 \%$ & $48 \%$ & $0 \%$ & $0 \%$ & $0 \%$ & $0 \%$ & $0 \%$ & $100 \%$ \\
\hline P16 & $0 \%$ & $0 \%$ & $20 \%$ & $19 \%$ & $0 \%$ & $1 \%$ & $0 \%$ & $10 \%$ & $50 \%$ & $0 \%$ & $0 \%$ & $0 \%$ & $0 \%$ & $0 \%$ & $100 \%$ \\
\hline P17 & $0 \%$ & $0 \%$ & $18 \%$ & $21 \%$ & $0 \%$ & $0 \%$ & $0 \%$ & $9 \%$ & $53 \%$ & $0 \%$ & $0 \%$ & $0 \%$ & $0 \%$ & $0 \%$ & $100 \%$ \\
\hline P18 & $0 \%$ & $0 \%$ & $17 \%$ & $22 \%$ & $0 \%$ & $0 \%$ & $0 \%$ & $6 \%$ & $55 \%$ & $0 \%$ & $0 \%$ & $0 \%$ & $0 \%$ & $0 \%$ & $100 \%$ \\
\hline P19 & $0 \%$ & $0 \%$ & $15 \%$ & $24 \%$ & $0 \%$ & $0 \%$ & $0 \%$ & $4 \%$ & $57 \%$ & $0 \%$ & $0 \%$ & $0 \%$ & $0 \%$ & $0 \%$ & $100 \%$ \\
\hline P20 & $0 \%$ & $0 \%$ & $13 \%$ & $25 \%$ & $0 \%$ & $0 \%$ & $0 \%$ & $2 \%$ & $60 \%$ & $0 \%$ & $0 \%$ & $0 \%$ & $0 \%$ & $0 \%$ & $100 \%$ \\
\hline P21 & $0 \%$ & $0 \%$ & $12 \%$ & $27 \%$ & $0 \%$ & $0 \%$ & $0 \%$ & $0 \%$ & $62 \%$ & $0 \%$ & $0 \%$ & $0 \%$ & $0 \%$ & $0 \%$ & $100 \%$ \\
\hline P22 & $0 \%$ & $0 \%$ & $8 \%$ & $29 \%$ & $0 \%$ & $0 \%$ & $0 \%$ & $0 \%$ & $63 \%$ & $0 \%$ & $0 \%$ & $0 \%$ & $0 \%$ & $0 \%$ & $100 \%$ \\
\hline P23 & $0 \%$ & $0 \%$ & $5 \%$ & $30 \%$ & $0 \%$ & $0 \%$ & $0 \%$ & $0 \%$ & $64 \%$ & $0 \%$ & $0 \%$ & $0 \%$ & $0 \%$ & $0 \%$ & $100 \%$ \\
\hline P24 & $0 \%$ & $0 \%$ & $2 \%$ & $32 \%$ & $0 \%$ & $0 \%$ & $0 \%$ & $0 \%$ & $65 \%$ & $0 \%$ & $0 \%$ & $0 \%$ & $0 \%$ & $0 \%$ & $100 \%$ \\
\hline P25 & $0 \%$ & $0 \%$ & $0 \%$ & $34 \%$ & $0 \%$ & $0 \%$ & $0 \%$ & $0 \%$ & $66 \%$ & $0 \%$ & $0 \%$ & $0 \%$ & $0 \%$ & $0 \%$ & $100 \%$ \\
\hline P26 & $0 \%$ & $0 \%$ & $0 \%$ & $37 \%$ & $0 \%$ & $0 \%$ & $0 \%$ & $0 \%$ & $63 \%$ & $0 \%$ & $0 \%$ & $0 \%$ & $0 \%$ & $0 \%$ & $100 \%$ \\
\hline P27 & $0 \%$ & $0 \%$ & $0 \%$ & $39 \%$ & $0 \%$ & $0 \%$ & $0 \%$ & $0 \%$ & $61 \%$ & $0 \%$ & $0 \%$ & $0 \%$ & $0 \%$ & $0 \%$ & $100 \%$ \\
\hline P28 & $0 \%$ & $0 \%$ & $0 \%$ & $42 \%$ & $0 \%$ & $0 \%$ & $0 \%$ & $0 \%$ & $58 \%$ & $0 \%$ & $0 \%$ & $0 \%$ & $0 \%$ & $0 \%$ & $100 \%$ \\
\hline P29 & $0 \%$ & $0 \%$ & $0 \%$ & $45 \%$ & $0 \%$ & $0 \%$ & $0 \%$ & $0 \%$ & $55 \%$ & $0 \%$ & $0 \%$ & $0 \%$ & $0 \%$ & $0 \%$ & $100 \%$ \\
\hline P30 & $0 \%$ & $0 \%$ & $0 \%$ & $47 \%$ & $0 \%$ & $0 \%$ & $0 \%$ & $0 \%$ & $53 \%$ & $0 \%$ & $0 \%$ & $0 \%$ & $0 \%$ & $0 \%$ & $100 \%$ \\
\hline P31 & $0 \%$ & $0 \%$ & $0 \%$ & $50 \%$ & $0 \%$ & $0 \%$ & $0 \%$ & $0 \%$ & $50 \%$ & $0 \%$ & $0 \%$ & $0 \%$ & $0 \%$ & $0 \%$ & $100 \%$ \\
\hline P32 & $0 \%$ & $0 \%$ & $0 \%$ & $53 \%$ & $0 \%$ & $0 \%$ & $0 \%$ & $0 \%$ & $47 \%$ & $0 \%$ & $0 \%$ & $0 \%$ & $0 \%$ & $0 \%$ & $100 \%$ \\
\hline P33 & $0 \%$ & $0 \%$ & $0 \%$ & $55 \%$ & $0 \%$ & $0 \%$ & $0 \%$ & $0 \%$ & $45 \%$ & $0 \%$ & $0 \%$ & $0 \%$ & $0 \%$ & $0 \%$ & $100 \%$ \\
\hline P34 & $0 \%$ & $0 \%$ & $0 \%$ & $58 \%$ & $0 \%$ & $0 \%$ & $0 \%$ & $0 \%$ & $42 \%$ & $0 \%$ & $0 \%$ & $0 \%$ & $0 \%$ & $0 \%$ & $100 \%$ \\
\hline P35 & $0 \%$ & $0 \%$ & $0 \%$ & $61 \%$ & $0 \%$ & $0 \%$ & $0 \%$ & $0 \%$ & $39 \%$ & $0 \%$ & $0 \%$ & $0 \%$ & $0 \%$ & $0 \%$ & $100 \%$ \\
\hline P36 & $0 \%$ & $0 \%$ & $0 \%$ & $63 \%$ & $0 \%$ & $0 \%$ & $0 \%$ & $0 \%$ & $37 \%$ & $0 \%$ & $0 \%$ & $0 \%$ & $0 \%$ & $0 \%$ & $100 \%$ \\
\hline P37 & $0 \%$ & $0 \%$ & $0 \%$ & $66 \%$ & $0 \%$ & $0 \%$ & $0 \%$ & $0 \%$ & $34 \%$ & $0 \%$ & $0 \%$ & $0 \%$ & $0 \%$ & $0 \%$ & $100 \%$ \\
\hline P38 & $0 \%$ & $0 \%$ & $0 \%$ & $68 \%$ & $0 \%$ & $0 \%$ & $0 \%$ & $0 \%$ & $32 \%$ & $0 \%$ & $0 \%$ & $0 \%$ & $0 \%$ & $0 \%$ & $100 \%$ \\
\hline P39 & $0 \%$ & $0 \%$ & $0 \%$ & $71 \%$ & $0 \%$ & $0 \%$ & $0 \%$ & $0 \%$ & $29 \%$ & $0 \%$ & $0 \%$ & $0 \%$ & $0 \%$ & $0 \%$ & $100 \%$ \\
\hline P40 & $0 \%$ & $0 \%$ & $0 \%$ & $74 \%$ & $0 \%$ & $0 \%$ & $0 \%$ & $0 \%$ & $26 \%$ & $0 \%$ & $0 \%$ & $0 \%$ & $0 \%$ & $0 \%$ & $100 \%$ \\
\hline P41 & $0 \%$ & $0 \%$ & $0 \%$ & $76 \%$ & $0 \%$ & $0 \%$ & $0 \%$ & $0 \%$ & $24 \%$ & $0 \%$ & $0 \%$ & $0 \%$ & $0 \%$ & $0 \%$ & $100 \%$ \\
\hline P42 & $0 \%$ & $0 \%$ & $0 \%$ & $79 \%$ & $0 \%$ & $0 \%$ & $0 \%$ & $0 \%$ & $21 \%$ & $0 \%$ & $0 \%$ & $0 \%$ & $0 \%$ & $0 \%$ & $100 \%$ \\
\hline P43 & $0 \%$ & $0 \%$ & $0 \%$ & $82 \%$ & $0 \%$ & $0 \%$ & $0 \%$ & $0 \%$ & $18 \%$ & $0 \%$ & $0 \%$ & $0 \%$ & $0 \%$ & $0 \%$ & $100 \%$ \\
\hline
\end{tabular}




\begin{tabular}{llllllllllllllll}
\hline P44 & $0 \%$ & $0 \%$ & $0 \%$ & $84 \%$ & $0 \%$ & $0 \%$ & $0 \%$ & $0 \%$ & $16 \%$ & $0 \%$ & $0 \%$ & $0 \%$ & $0 \%$ & $\mathbf{0 \%}$ & $100 \%$ \\
P45 & $0 \%$ & $0 \%$ & $0 \%$ & $87 \%$ & $0 \%$ & $0 \%$ & $0 \%$ & $0 \%$ & $13 \%$ & $0 \%$ & $0 \%$ & $0 \%$ & $0 \%$ & $\mathbf{0 \%}$ & $100 \%$ \\
$\mathbf{P 4 6}$ & $0 \%$ & $0 \%$ & $0 \%$ & $89 \%$ & $0 \%$ & $0 \%$ & $0 \%$ & $0 \%$ & $11 \%$ & $0 \%$ & $0 \%$ & $0 \%$ & $0 \%$ & $\mathbf{0 \%}$ & $100 \%$ \\
$\mathbf{P 4 7}$ & $0 \%$ & $0 \%$ & $0 \%$ & $92 \%$ & $0 \%$ & $0 \%$ & $0 \%$ & $0 \%$ & $8 \%$ & $0 \%$ & $0 \%$ & $0 \%$ & $0 \%$ & $\mathbf{0 \%}$ & $100 \%$ \\
$\mathbf{P 4 8}$ & $0 \%$ & $0 \%$ & $0 \%$ & $95 \%$ & $0 \%$ & $0 \%$ & $0 \%$ & $0 \%$ & $5 \%$ & $0 \%$ & $0 \%$ & $0 \%$ & $0 \%$ & $\mathbf{0 \%}$ & $100 \%$ \\
$\mathbf{P 4 9}$ & $0 \%$ & $0 \%$ & $0 \%$ & $97 \%$ & $0 \%$ & $0 \%$ & $0 \%$ & $0 \%$ & $3 \%$ & $0 \%$ & $0 \%$ & $0 \%$ & $0 \%$ & $\mathbf{0 \%}$ & $100 \%$ \\
P50 & $0 \%$ & $0 \%$ & $0 \%$ & $100 \%$ & $0 \%$ & $0 \%$ & $0 \%$ & $0 \%$ & $0 \%$ & $0 \%$ & $0 \%$ & $0 \%$ & $0 \%$ & $\mathbf{0 \%}$ & $100 \%$ \\
\hline
\end{tabular}

Table 15. Portfolio weights by SemiVariance model

\begin{tabular}{|c|c|c|c|c|c|c|c|c|c|c|c|c|c|c|c|}
\hline Portfolios & Namibia $N$ & gerian C & Gongo & $\begin{array}{c}\text { Cote } \\
\text { D'Ivoire } \\
\end{array}$ & Egypt $\Lambda$ & Morrocco T & Tunisia & Botswana 1 & Mauritius & Kenya $L$ & Uganda & Zambia & $\begin{array}{l}\text { South } \\
\text { Africa }\end{array}$ & Euro & $\begin{array}{c}\text { Total } \\
\text { PortfolioWeight } \\
\end{array}$ \\
\hline MPV & $0 \%$ & $0 \%$ & $38 \%$ & $2 \%$ & $0 \%$ & $0 \%$ & $26 \%$ & $12 \%$ & $13 \%$ & $2 \%$ & $0 \%$ & $2 \%$ & $0 \%$ & $5 \%$ & $100 \%$ \\
\hline $\mathbf{P 2}$ & $0 \%$ & $0 \%$ & $38 \%$ & $2 \%$ & $0 \%$ & $0 \%$ & $27 \%$ & $12 \%$ & $13 \%$ & $1 \%$ & $0 \%$ & $2 \%$ & $0 \%$ & $5 \%$ & $100 \%$ \\
\hline P3 & $0 \%$ & $0 \%$ & $38 \%$ & $3 \%$ & $0 \%$ & $0 \%$ & $27 \%$ & $11 \%$ & $13 \%$ & $0 \%$ & $0 \%$ & $3 \%$ & $0 \%$ & $6 \%$ & $100 \%$ \\
\hline P4 & $0 \%$ & $0 \%$ & $38 \%$ & $4 \%$ & $0 \%$ & $0 \%$ & $27 \%$ & $9 \%$ & $12 \%$ & $0 \%$ & $0 \%$ & $4 \%$ & $0 \%$ & $5 \%$ & $100 \%$ \\
\hline P5 & $0 \%$ & $0 \%$ & $39 \%$ & $5 \%$ & $0 \%$ & $0 \%$ & $27 \%$ & $7 \%$ & $12 \%$ & $0 \%$ & $0 \%$ & $4 \%$ & $0 \%$ & $6 \%$ & $100 \%$ \\
\hline P6 & $0 \%$ & $0 \%$ & $39 \%$ & $7 \%$ & $0 \%$ & $0 \%$ & $27 \%$ & $4 \%$ & $11 \%$ & $0 \%$ & $0 \%$ & $5 \%$ & $0 \%$ & $6 \%$ & $100 \%$ \\
\hline P7 & $0 \%$ & $0 \%$ & $39 \%$ & $8 \%$ & $0 \%$ & $0 \%$ & $27 \%$ & $2 \%$ & $11 \%$ & $0 \%$ & $0 \%$ & $6 \%$ & $0 \%$ & $6 \%$ & $100 \%$ \\
\hline P8 & $0 \%$ & $0 \%$ & $40 \%$ & $9 \%$ & $0 \%$ & $0 \%$ & $27 \%$ & $0 \%$ & $10 \%$ & $0 \%$ & $0 \%$ & $7 \%$ & $0 \%$ & $6 \%$ & $100 \%$ \\
\hline P9 & $0 \%$ & $0 \%$ & $40 \%$ & $11 \%$ & $0 \%$ & $0 \%$ & $26 \%$ & $0 \%$ & $9 \%$ & $0 \%$ & $0 \%$ & $8 \%$ & $0 \%$ & $6 \%$ & $100 \%$ \\
\hline P10 & $0 \%$ & $0 \%$ & $39 \%$ & $12 \%$ & $0 \%$ & $0 \%$ & $25 \%$ & $0 \%$ & $8 \%$ & $0 \%$ & $0 \%$ & $8 \%$ & $0 \%$ & 6\% & $100 \%$ \\
\hline P11 & $0 \%$ & $0 \%$ & $39 \%$ & $14 \%$ & $0 \%$ & $0 \%$ & $24 \%$ & $0 \%$ & $7 \%$ & $0 \%$ & $0 \%$ & $9 \%$ & $0 \%$ & $6 \%$ & $100 \%$ \\
\hline P12 & $0 \%$ & $0 \%$ & $39 \%$ & $16 \%$ & $0 \%$ & $0 \%$ & $23 \%$ & $0 \%$ & $5 \%$ & $0 \%$ & $0 \%$ & $6 \quad 10 \%$ & $0 \%$ & $7 \%$ & $100 \%$ \\
\hline P13 & $0 \%$ & $0 \%$ & $39 \%$ & $18 \%$ & $0 \%$ & $0 \%$ & $22 \%$ & $0 \%$ & $4 \%$ & $0 \%$ & $0 \%$ & $11 \%$ & $0 \%$ & $6 \%$ & $100 \%$ \\
\hline P14 & $0 \%$ & $0 \%$ & $38 \%$ & $19 \%$ & $0 \%$ & $0 \%$ & $21 \%$ & $0 \%$ & $2 \%$ & $0 \%$ & $0 \%$ & $6 \quad 12 \%$ & $0 \%$ & $6 \%$ & $100 \%$ \\
\hline P15 & $0 \%$ & $0 \%$ & $38 \%$ & $21 \%$ & $1 \%$ & $0 \%$ & $20 \%$ & $0 \%$ & $1 \%$ & $0 \%$ & $0 \%$ & b $12 \%$ & $0 \%$ & $6 \%$ & $100 \%$ \\
\hline P16 & $0 \%$ & $0 \%$ & $38 \%$ & $23 \%$ & $1 \%$ & $0 \%$ & $18 \%$ & $0 \%$ & $0 \%$ & $0 \%$ & $0 \%$ & $13 \%$ & $0 \%$ & $67 \%$ & $100 \%$ \\
\hline P17 & $0 \%$ & $0 \%$ & $37 \%$ & $25 \%$ & $1 \%$ & $0 \%$ & $16 \%$ & $0 \%$ & $0 \%$ & $0 \%$ & $0 \%$ & $14 \%$ & $0 \%$ & $6 \%$ & $100 \%$ \\
\hline P18 & $0 \%$ & $0 \%$ & $36 \%$ & $27 \%$ & $1 \%$ & $0 \%$ & $14 \%$ & $0 \%$ & $0 \%$ & $0 \%$ & $0 \%$ & $14 \%$ & $0 \%$ & $6 \%$ & $100 \%$ \\
\hline P19 & $0 \%$ & $0 \%$ & $36 \%$ & $29 \%$ & $1 \%$ & $0 \%$ & $12 \%$ & $0 \%$ & $0 \%$ & $0 \%$ & $0 \%$ & $15 \%$ & $0 \%$ & $6 \%$ & $100 \%$ \\
\hline P20 & $0 \%$ & $0 \%$ & $35 \%$ & $31 \%$ & $1 \%$ & $0 \%$ & $10 \%$ & $0 \%$ & $0 \%$ & $0 \%$ & $0 \%$ & $15 \%$ & $0 \%$ & $67 \%$ & $100 \%$ \\
\hline P21 & $0 \%$ & $0 \%$ & $34 \%$ & $33 \%$ & $1 \%$ & $0 \%$ & $8 \%$ & $0 \%$ & $0 \%$ & $0 \%$ & $0 \%$ & $16 \%$ & $0 \%$ & $67 \%$ & $100 \%$ \\
\hline P22 & $0 \%$ & $0 \%$ & $34 \%$ & $35 \%$ & $1 \%$ & $0 \%$ & $6 \%$ & $0 \%$ & $0 \%$ & $0 \%$ & $0 \%$ & $17 \%$ & $0 \%$ & $6 \%$ & $100 \%$ \\
\hline P23 & $0 \%$ & $0 \%$ & $33 \%$ & $36 \%$ & $2 \%$ & $0 \%$ & $5 \%$ & $0 \%$ & $0 \%$ & $0 \%$ & $0 \%$ & $17 \%$ & $0 \%$ & $6 \%$ & $100 \%$ \\
\hline P24 & $0 \%$ & $0 \%$ & $32 \%$ & $38 \%$ & $2 \%$ & $0 \%$ & $3 \%$ & $0 \%$ & $0 \%$ & $0 \%$ & $0 \%$ & $18 \%$ & $0 \%$ & $67 \%$ & $100 \%$ \\
\hline P25 & $0 \%$ & $0 \%$ & $32 \%$ & $40 \%$ & $2 \%$ & $0 \%$ & $1 \%$ & $0 \%$ & $0 \%$ & $0 \%$ & $0 \%$ & $19 \%$ & $0 \%$ & $67 \%$ & $100 \%$ \\
\hline P26 & $0 \%$ & $0 \%$ & $30 \%$ & $42 \%$ & $2 \%$ & $0 \%$ & $0 \%$ & $0 \%$ & $0 \%$ & $0 \%$ & $0 \%$ & $19 \%$ & $0 \%$ & $6 \%$ & $100 \%$ \\
\hline P27 & $0 \%$ & $0 \%$ & $28 \%$ & $44 \%$ & $2 \%$ & $0 \%$ & $0 \%$ & $0 \%$ & $0 \%$ & $0 \%$ & $0 \%$ & $19 \%$ & $0 \%$ & $6 \%$ & $100 \%$ \\
\hline P28 & $0 \%$ & $0 \%$ & $27 \%$ & $47 \%$ & $2 \%$ & $0 \%$ & $0 \%$ & $0 \%$ & $0 \%$ & $0 \%$ & $0 \%$ & $20 \%$ & $0 \%$ & $5 \%$ & $100 \%$ \\
\hline P29 & $0 \%$ & $0 \%$ & $25 \%$ & $49 \%$ & $2 \%$ & $0 \%$ & $0 \%$ & $0 \%$ & $0 \%$ & $0 \%$ & $0 \%$ & $20 \%$ & $0 \%$ & $65 \%$ & $100 \%$ \\
\hline P30 & $0 \%$ & $0 \%$ & $23 \%$ & $51 \%$ & $2 \%$ & $0 \%$ & $0 \%$ & $0 \%$ & $0 \%$ & $0 \%$ & $0 \%$ & $21 \%$ & $0 \%$ & $4 \%$ & $100 \%$ \\
\hline P31 & $0 \%$ & $0 \%$ & $21 \%$ & $53 \%$ & $1 \%$ & $0 \%$ & $0 \%$ & $0 \%$ & $0 \%$ & $0 \%$ & $0 \%$ & $21 \%$ & $0 \%$ & $4 \%$ & $100 \%$ \\
\hline P32 & $0 \%$ & $0 \%$ & $19 \%$ & $55 \%$ & $1 \%$ & $0 \%$ & $0 \%$ & $0 \%$ & $0 \%$ & $0 \%$ & $0 \%$ & $22 \%$ & $0 \%$ & $63 \%$ & $100 \%$ \\
\hline P33 & $0 \%$ & $0 \%$ & $17 \%$ & $57 \%$ & $1 \%$ & $0 \%$ & $0 \%$ & $0 \%$ & $0 \%$ & $0 \%$ & $0 \%$ & $22 \%$ & $0 \%$ & $6 \quad 2 \%$ & $100 \%$ \\
\hline P34 & $0 \%$ & $0 \%$ & $15 \%$ & $59 \%$ & $1 \%$ & $0 \%$ & $0 \%$ & $0 \%$ & $0 \%$ & $0 \%$ & $0 \%$ & $22 \%$ & $0 \%$ & $62 \%$ & $100 \%$ \\
\hline P35 & $0 \%$ & $0 \%$ & $13 \%$ & $62 \%$ & $1 \%$ & $0 \%$ & $0 \%$ & $0 \%$ & $0 \%$ & $0 \%$ & $0 \%$ & $23 \%$ & $0 \%$ & 6 $1 \%$ & $100 \%$ \\
\hline P36 & $0 \%$ & $0 \%$ & $11 \%$ & $64 \%$ & $1 \%$ & $0 \%$ & $0 \%$ & $0 \%$ & $0 \%$ & $0 \%$ & $0 \%$ & $23 \%$ & $0 \%$ & $6 \quad 1 \%$ & $100 \%$ \\
\hline P37 & $0 \%$ & $0 \%$ & $9 \%$ & $66 \%$ & $1 \%$ & $0 \%$ & $0 \%$ & $0 \%$ & $0 \%$ & $0 \%$ & $0 \%$ & $24 \%$ & $0 \%$ & $6 \%$ & $100 \%$ \\
\hline P38 & $0 \%$ & $0 \%$ & $7 \%$ & $68 \%$ & $1 \%$ & $0 \%$ & $0 \%$ & $0 \%$ & $0 \%$ & $0 \%$ & $0 \%$ & $24 \%$ & $0 \%$ & $6 \%$ & $100 \%$ \\
\hline P39 & $0 \%$ & $0 \%$ & $5 \%$ & $70 \%$ & $1 \%$ & $0 \%$ & $0 \%$ & $0 \%$ & $0 \%$ & $0 \%$ & $0 \%$ & $24 \%$ & $0 \%$ & $6 \%$ & $100 \%$ \\
\hline P40 & $0 \%$ & $0 \%$ & $3 \%$ & $72 \%$ & $1 \%$ & $0 \%$ & $0 \%$ & $0 \%$ & $0 \%$ & $0 \%$ & $0 \%$ & $25 \%$ & $0 \%$ & $6 \%$ & $100 \%$ \\
\hline P41 & $0 \%$ & $0 \%$ & $0 \%$ & $74 \%$ & $0 \%$ & $0 \%$ & $0 \%$ & $0 \%$ & $0 \%$ & $0 \%$ & $0 \%$ & $25 \%$ & $0 \%$ & $6 \%$ & $100 \%$ \\
\hline P42 & $0 \%$ & $0 \%$ & $0 \%$ & $77 \%$ & $0 \%$ & $0 \%$ & $0 \%$ & $0 \%$ & $0 \%$ & $0 \%$ & $0 \%$ & $23 \%$ & $0 \%$ & $0 \%$ & $100 \%$ \\
\hline P43 & $0 \%$ & $0 \%$ & $0 \%$ & $80 \%$ & $0 \%$ & $0 \%$ & $0 \%$ & $0 \%$ & $0 \%$ & $0 \%$ & $0 \%$ & $6 \quad 20 \%$ & $0 \%$ & $6 \%$ & $100 \%$ \\
\hline P44 & $0 \%$ & $0 \%$ & $0 \%$ & $83 \%$ & $0 \%$ & $0 \%$ & $0 \%$ & $0 \%$ & $0 \%$ & $0 \%$ & $0 \%$ & $17 \%$ & $0 \%$ & $6 \%$ & $100 \%$ \\
\hline P45 & $0 \%$ & $0 \%$ & $0 \%$ & $86 \%$ & $0 \%$ & $0 \%$ & $0 \%$ & $0 \%$ & $0 \%$ & $0 \%$ & $0 \%$ & $14 \%$ & $0 \%$ & $6 \%$ & $100 \%$ \\
\hline P46 & $0 \%$ & $0 \%$ & $0 \%$ & $88 \%$ & $0 \%$ & $0 \%$ & $0 \%$ & $0 \%$ & $0 \%$ & $0 \%$ & $0 \%$ & $12 \%$ & $0 \%$ & $0 \%$ & $100 \%$ \\
\hline P47 & $0 \%$ & $0 \%$ & $0 \%$ & $91 \%$ & $0 \%$ & $0 \%$ & $0 \%$ & $0 \%$ & $0 \%$ & $0 \%$ & $0 \%$ & $9 \%$ & $0 \%$ & $6 \%$ & $100 \%$ \\
\hline P48 & $0 \%$ & $0 \%$ & $0 \%$ & $94 \%$ & $0 \%$ & $0 \%$ & $0 \%$ & $0 \%$ & $0 \%$ & $0 \%$ & $0 \%$ & $6 \%$ & $0 \%$ & $0 \%$ & $100 \%$ \\
\hline P49 & $0 \%$ & $0 \%$ & $0 \%$ & $97 \%$ & $0 \%$ & $0 \%$ & $0 \%$ & $0 \%$ & $0 \%$ & $0 \%$ & $0 \%$ & $3 \%$ & $0 \%$ & $6 \%$ & $100 \%$ \\
\hline P50 & $0 \%$ & $0 \%$ & $0 \%$ & $100 \%$ & $0 \%$ & $0 \%$ & $0 \%$ & $0 \%$ & $0 \%$ & $0 \%$ & $0 \%$ & $0 \%$ & $0 \%$ & $0 \%$ & $100 \%$ \\
\hline
\end{tabular}


Table 16. Portfolio weights by Mean Absolute Deviation model

\begin{tabular}{|c|c|c|c|c|c|c|c|c|c|c|c|c|c|c|c|}
\hline Portfolios & Vamibia & Vigerian & Gongo & $\begin{array}{c}\text { Cote } \\
\text { D'Ivoire }\end{array}$ & Egypt & Morrocco & Tunisia & Botswana & Mauritius & Kenya & Uganda & Zambia & $\begin{array}{l}\text { South } \\
\text { Africa }\end{array}$ & Europe & $\begin{array}{c}\text { Total } \\
e_{\text {PortfolioWeight }} \\
\end{array}$ \\
\hline MPV & $0 \%$ & $0 \%$ & $92 \%$ & $0 \%$ & $0 \%$ & $0 \%$ & $2 \%$ & $1 \%$ & $2 \%$ & $0 \%$ & $0 \%$ & $0 \%$ & $0 \%$ & $2 \%$ & $100 \%$ \\
\hline $\mathbf{P 2}$ & $0 \%$ & $0 \%$ & $94 \%$ & $0 \%$ & $0 \%$ & $0 \%$ & $1 \%$ & $0 \%$ & $1 \%$ & $0 \%$ & $0 \%$ & $1 \%$ & $0 \%$ & $2 \%$ & $100 \%$ \\
\hline P3 & $0 \%$ & $0 \%$ & $93 \%$ & $2 \%$ & $0 \%$ & $0 \%$ & $1 \%$ & $0 \%$ & $0 \%$ & $0 \%$ & $0 \%$ & $1 \%$ & $0 \%$ & $2 \%$ & $100 \%$ \\
\hline P4 & $0 \%$ & $0 \%$ & $91 \%$ & $4 \%$ & $0 \%$ & $0 \%$ & $1 \%$ & $0 \%$ & $0 \%$ & $0 \%$ & $0 \%$ & $2 \%$ & $0 \%$ & $1 \%$ & $100 \%$ \\
\hline P5 & $0 \%$ & $0 \%$ & $89 \%$ & $6 \%$ & $0 \%$ & $0 \%$ & $1 \%$ & $0 \%$ & $0 \%$ & $0 \%$ & $0 \%$ & $2 \%$ & $0 \%$ & $1 \%$ & $100 \%$ \\
\hline P6 & $0 \%$ & $0 \%$ & $87 \%$ & $8 \%$ & $0 \%$ & $0 \%$ & $0 \%$ & $0 \%$ & $0 \%$ & $0 \%$ & $0 \%$ & $3 \%$ & $0 \%$ & $1 \%$ & $100 \%$ \\
\hline P7 & $0 \%$ & $0 \%$ & $85 \%$ & $10 \%$ & $1 \%$ & $0 \%$ & $0 \%$ & $0 \%$ & $0 \%$ & $0 \%$ & $1 \%$ & $3 \%$ & $0 \%$ & $1 \%$ & $100 \%$ \\
\hline P8 & $0 \%$ & $0 \%$ & $82 \%$ & $12 \%$ & $1 \%$ & $0 \%$ & $1 \%$ & $0 \%$ & $0 \%$ & $0 \%$ & $0 \%$ & $3 \%$ & $0 \%$ & $1 \%$ & $100 \%$ \\
\hline P9 & $0 \%$ & $0 \%$ & $79 \%$ & $14 \%$ & $1 \%$ & $0 \%$ & $0 \%$ & $0 \%$ & $0 \%$ & $0 \%$ & $1 \%$ & $4 \%$ & $0 \%$ & $1 \%$ & $100 \%$ \\
\hline P10 & $0 \%$ & $0 \%$ & $77 \%$ & $16 \%$ & $1 \%$ & $0 \%$ & $1 \%$ & $0 \%$ & $0 \%$ & $0 \%$ & $1 \%$ & $4 \%$ & $0 \%$ & $1 \%$ & $100 \%$ \\
\hline P11 & $0 \%$ & $0 \%$ & $75 \%$ & $18 \%$ & $1 \%$ & $0 \%$ & $0 \%$ & $0 \%$ & $0 \%$ & $0 \%$ & $1 \%$ & $4 \%$ & $0 \%$ & $1 \%$ & $100 \%$ \\
\hline P12 & $0 \%$ & $0 \%$ & $73 \%$ & $20 \%$ & $1 \%$ & $0 \%$ & $0 \%$ & $0 \%$ & $0 \%$ & $0 \%$ & $1 \%$ & $5 \%$ & $0 \%$ & $1 \%$ & $100 \%$ \\
\hline P13 & $0 \%$ & $0 \%$ & $70 \%$ & $22 \%$ & $1 \%$ & $0 \%$ & $0 \%$ & $0 \%$ & $0 \%$ & $0 \%$ & $1 \%$ & $5 \%$ & $0 \%$ & $1 \%$ & $100 \%$ \\
\hline P14 & $0 \%$ & $0 \%$ & $67 \%$ & $24 \%$ & $1 \%$ & $0 \%$ & $1 \%$ & $0 \%$ & $0 \%$ & $0 \%$ & $1 \%$ & $5 \%$ & $0 \%$ & $1 \%$ & $100 \%$ \\
\hline P15 & $0 \%$ & $0 \%$ & $65 \%$ & $26 \%$ & $1 \%$ & $0 \%$ & $1 \%$ & $0 \%$ & $0 \%$ & $0 \%$ & $1 \%$ & $5 \%$ & $0 \%$ & $1 \%$ & $100 \%$ \\
\hline P16 & $0 \%$ & $0 \%$ & $62 \%$ & $28 \%$ & $1 \%$ & $0 \%$ & $1 \%$ & $0 \%$ & $0 \%$ & $0 \%$ & $1 \%$ & $6 \%$ & $0 \%$ & $1 \%$ & $100 \%$ \\
\hline P17 & $0 \%$ & $0 \%$ & $60 \%$ & $30 \%$ & $1 \%$ & $0 \%$ & $1 \%$ & $0 \%$ & $0 \%$ & $0 \%$ & $1 \%$ & $6 \%$ & $0 \%$ & $1 \%$ & $100 \%$ \\
\hline P18 & $0 \%$ & $0 \%$ & $57 \%$ & $32 \%$ & $1 \%$ & $0 \%$ & $1 \%$ & $0 \%$ & $0 \%$ & $0 \%$ & $1 \%$ & $6 \%$ & $0 \%$ & $1 \%$ & $100 \%$ \\
\hline P19 & $0 \%$ & $0 \%$ & $54 \%$ & $34 \%$ & $1 \%$ & $0 \%$ & $2 \%$ & $0 \%$ & $0 \%$ & $0 \%$ & $1 \%$ & $6 \%$ & $0 \%$ & $2 \%$ & $100 \%$ \\
\hline P20 & $0 \%$ & $0 \%$ & $52 \%$ & $36 \%$ & $1 \%$ & $0 \%$ & $2 \%$ & $0 \%$ & $0 \%$ & $0 \%$ & $1 \%$ & $6 \%$ & $0 \%$ & $2 \%$ & $100 \%$ \\
\hline P21 & $0 \%$ & $0 \%$ & $49 \%$ & $38 \%$ & $1 \%$ & $0 \%$ & $3 \%$ & $0 \%$ & $0 \%$ & $0 \%$ & $1 \%$ & $7 \%$ & $0 \%$ & $2 \%$ & $100 \%$ \\
\hline P22 & $0 \%$ & $0 \%$ & $46 \%$ & $40 \%$ & $2 \%$ & $0 \%$ & $3 \%$ & $0 \%$ & $0 \%$ & $0 \%$ & $1 \%$ & $7 \%$ & $0 \%$ & $2 \%$ & $100 \%$ \\
\hline P23 & $0 \%$ & $0 \%$ & $44 \%$ & $42 \%$ & $1 \%$ & $0 \%$ & $3 \%$ & $0 \%$ & $0 \%$ & $0 \%$ & $1 \%$ & $7 \%$ & $0 \%$ & $2 \%$ & $100 \%$ \\
\hline P24 & $0 \%$ & $0 \%$ & $41 \%$ & $44 \%$ & $2 \%$ & $0 \%$ & $4 \%$ & $0 \%$ & $0 \%$ & $0 \%$ & $1 \%$ & $7 \%$ & $0 \%$ & $2 \%$ & $100 \%$ \\
\hline P25 & $0 \%$ & $0 \%$ & $39 \%$ & $46 \%$ & $2 \%$ & $0 \%$ & $4 \%$ & $0 \%$ & $0 \%$ & $0 \%$ & $1 \%$ & $7 \%$ & $0 \%$ & $2 \%$ & $100 \%$ \\
\hline P26 & $0 \%$ & $0 \%$ & $36 \%$ & $48 \%$ & $2 \%$ & $0 \%$ & $4 \%$ & $0 \%$ & $0 \%$ & $0 \%$ & $1 \%$ & $7 \%$ & $0 \%$ & $2 \%$ & $100 \%$ \\
\hline P27 & $0 \%$ & $0 \%$ & $35 \%$ & $50 \%$ & $2 \%$ & $0 \%$ & $4 \%$ & $0 \%$ & $0 \%$ & $0 \%$ & $1 \%$ & $7 \%$ & $0 \%$ & $2 \%$ & $100 \%$ \\
\hline P28 & $0 \%$ & $0 \%$ & $33 \%$ & $52 \%$ & $2 \%$ & $0 \%$ & $3 \%$ & $0 \%$ & $0 \%$ & $0 \%$ & $1 \%$ & $7 \%$ & $0 \%$ & $2 \%$ & $100 \%$ \\
\hline P29 & $0 \%$ & $0 \%$ & $32 \%$ & $54 \%$ & $2 \%$ & $0 \%$ & $3 \%$ & $0 \%$ & $0 \%$ & $0 \%$ & $0 \%$ & $8 \%$ & $0 \%$ & $2 \%$ & $100 \%$ \\
\hline P30 & $0 \%$ & $0 \%$ & $29 \%$ & $56 \%$ & $2 \%$ & $0 \%$ & $3 \%$ & $0 \%$ & $0 \%$ & $0 \%$ & $0 \%$ & $8 \%$ & $0 \%$ & $2 \%$ & $100 \%$ \\
\hline P31 & $0 \%$ & $0 \%$ & $29 \%$ & $58 \%$ & $2 \%$ & $0 \%$ & $2 \%$ & $0 \%$ & $0 \%$ & $0 \%$ & $0 \%$ & $8 \%$ & $0 \%$ & $1 \%$ & $100 \%$ \\
\hline P32 & $0 \%$ & $0 \%$ & $28 \%$ & $60 \%$ & $2 \%$ & $0 \%$ & $1 \%$ & $0 \%$ & $0 \%$ & $0 \%$ & $0 \%$ & $8 \%$ & $0 \%$ & $1 \%$ & $100 \%$ \\
\hline P33 & $0 \%$ & $0 \%$ & $26 \%$ & $62 \%$ & $2 \%$ & $0 \%$ & $1 \%$ & $0 \%$ & $0 \%$ & $0 \%$ & $0 \%$ & $9 \%$ & $0 \%$ & $1 \%$ & $100 \%$ \\
\hline P34 & $0 \%$ & $0 \%$ & $24 \%$ & $64 \%$ & $2 \%$ & $0 \%$ & $1 \%$ & $0 \%$ & $0 \%$ & $0 \%$ & $0 \%$ & $8 \%$ & $0 \%$ & $1 \%$ & $100 \%$ \\
\hline P35 & $0 \%$ & $0 \%$ & $23 \%$ & $66 \%$ & $2 \%$ & $0 \%$ & $0 \%$ & $0 \%$ & $0 \%$ & $0 \%$ & $0 \%$ & $9 \%$ & $0 \%$ & $1 \%$ & $100 \%$ \\
\hline P36 & $0 \%$ & $0 \%$ & $20 \%$ & $68 \%$ & $2 \%$ & $0 \%$ & $0 \%$ & $0 \%$ & $0 \%$ & $0 \%$ & $0 \%$ & $9 \%$ & $0 \%$ & $1 \%$ & $100 \%$ \\
\hline P37 & $0 \%$ & $0 \%$ & $18 \%$ & $70 \%$ & $2 \%$ & $0 \%$ & $0 \%$ & $0 \%$ & $0 \%$ & $0 \%$ & $0 \%$ & $9 \%$ & $0 \%$ & $1 \%$ & $100 \%$ \\
\hline P38 & $0 \%$ & $0 \%$ & $16 \%$ & $73 \%$ & $2 \%$ & $0 \%$ & $0 \%$ & $0 \%$ & $0 \%$ & $0 \%$ & $0 \%$ & $9 \%$ & $0 \%$ & $1 \%$ & $100 \%$ \\
\hline P39 & $0 \%$ & $0 \%$ & $14 \%$ & $75 \%$ & $2 \%$ & $0 \%$ & $0 \%$ & $0 \%$ & $0 \%$ & $0 \%$ & $0 \%$ & $9 \%$ & $0 \%$ & $1 \%$ & $100 \%$ \\
\hline P40 & $0 \%$ & $0 \%$ & $12 \%$ & $77 \%$ & $2 \%$ & $0 \%$ & $0 \%$ & $0 \%$ & $0 \%$ & $0 \%$ & $0 \%$ & $9 \%$ & $0 \%$ & $0 \%$ & $100 \%$ \\
\hline P41 & $0 \%$ & $0 \%$ & $10 \%$ & $79 \%$ & $2 \%$ & $0 \%$ & $0 \%$ & $0 \%$ & $0 \%$ & $0 \%$ & $0 \%$ & $9 \%$ & $0 \%$ & $0 \%$ & $100 \%$ \\
\hline P42 & $0 \%$ & $0 \%$ & $8 \%$ & $81 \%$ & $2 \%$ & $0 \%$ & $0 \%$ & $0 \%$ & $0 \%$ & $0 \%$ & $0 \%$ & $9 \%$ & $0 \%$ & $0 \%$ & $100 \%$ \\
\hline P43 & $0 \%$ & $0 \%$ & $6 \%$ & $83 \%$ & $2 \%$ & $0 \%$ & $0 \%$ & $0 \%$ & $0 \%$ & $0 \%$ & $0 \%$ & $9 \%$ & $0 \%$ & $0 \%$ & $100 \%$ \\
\hline P44 & $0 \%$ & $0 \%$ & $4 \%$ & $85 \%$ & $1 \%$ & $0 \%$ & $0 \%$ & $0 \%$ & $0 \%$ & $0 \%$ & $0 \%$ & $9 \%$ & $0 \%$ & $0 \%$ & $100 \%$ \\
\hline P45 & $0 \%$ & $0 \%$ & $2 \%$ & $87 \%$ & $1 \%$ & $0 \%$ & $0 \%$ & $0 \%$ & $0 \%$ & $0 \%$ & $0 \%$ & $9 \%$ & $0 \%$ & $0 \%$ & $100 \%$ \\
\hline P46 & $0 \%$ & $0 \%$ & $1 \%$ & $89 \%$ & $0 \%$ & $0 \%$ & $0 \%$ & $0 \%$ & $0 \%$ & $0 \%$ & $0 \%$ & $9 \%$ & $0 \%$ & $0 \%$ & $100 \%$ \\
\hline P47 & $0 \%$ & $0 \%$ & $0 \%$ & $92 \%$ & $0 \%$ & $0 \%$ & $0 \%$ & $0 \%$ & $0 \%$ & $0 \%$ & $0 \%$ & $8 \%$ & $0 \%$ & $0 \%$ & $100 \%$ \\
\hline P48 & $0 \%$ & $0 \%$ & $0 \%$ & $94 \%$ & $0 \%$ & $0 \%$ & $0 \%$ & $0 \%$ & $0 \%$ & $0 \%$ & $0 \%$ & $6 \%$ & $0 \%$ & $0 \%$ & $100 \%$ \\
\hline P49 & $0 \%$ & $0 \%$ & $0 \%$ & $97 \%$ & $0 \%$ & $0 \%$ & $0 \%$ & $0 \%$ & $0 \%$ & $0 \%$ & $0 \%$ & $3 \%$ & $0 \%$ & $0 \%$ & $100 \%$ \\
\hline P50 & $0 \%$ & $0 \%$ & $0 \%$ & $100 \%$ & $0 \%$ & $0 \%$ & $0 \%$ & $0 \%$ & $0 \%$ & $0 \%$ & $0 \%$ & $0 \%$ & $0 \%$ & $0 \%$ & $100 \%$ \\
\hline
\end{tabular}


Table 17. Portfolio weights by Mean Filtered Historical Simulation model

\begin{tabular}{|c|c|c|c|c|c|c|c|c|c|c|c|c|c|c|c|}
\hline Portfolios & Namibia & Vigerian & Gongo & $\begin{array}{c}\text { Cote } \\
\text { D'Ivoire }\end{array}$ & Egypt & Morrocco & Tunisia & Botswana & Mauritius & Kenya & Uganda & Zambia & $\begin{array}{c}\text { South } \\
\text { Africa }\end{array}$ & Europe & $\begin{array}{c}\text { Total } \\
\text { PortfolioWeight }\end{array}$ \\
\hline MPV & $3 \%$ & $1 \%$ & $3 \%$ & $6 \%$ & $3 \%$ & $2 \%$ & $3 \%$ & $3 \%$ & $3 \%$ & $2 \%$ & $3 \%$ & $3 \%$ & $3 \%$ & $62 \%$ & $100 \%$ \\
\hline P2 & $2 \%$ & $1 \%$ & $3 \%$ & $5 \%$ & $3 \%$ & $3 \%$ & $3 \%$ & $3 \%$ & $3 \%$ & $2 \%$ & $3 \%$ & $4 \%$ & $3 \%$ & $62 \%$ & $100 \%$ \\
\hline P3 & $2 \%$ & $2 \%$ & $3 \%$ & $6 \%$ & $3 \%$ & $3 \%$ & $3 \%$ & $3 \%$ & $3 \%$ & $2 \%$ & $3 \%$ & $3 \%$ & $3 \%$ & $61 \%$ & $100 \%$ \\
\hline P4 & $2 \%$ & $1 \%$ & $3 \%$ & $5 \%$ & $3 \%$ & $2 \%$ & $3 \%$ & $3 \%$ & $3 \%$ & $2 \%$ & $3 \%$ & $4 \%$ & $3 \%$ & $63 \%$ & $100 \%$ \\
\hline P5 & $2 \%$ & $1 \%$ & $5 \%$ & $5 \%$ & $2 \%$ & $3 \%$ & $3 \%$ & $3 \%$ & $3 \%$ & $3 \%$ & $3 \%$ & $3 \%$ & $3 \%$ & $61 \%$ & $100 \%$ \\
\hline P6 & $3 \%$ & $2 \%$ & $3 \%$ & $5 \%$ & $3 \%$ & $3 \%$ & $3 \%$ & $3 \%$ & $3 \%$ & $2 \%$ & $3 \%$ & $3 \%$ & $3 \%$ & $61 \%$ & $100 \%$ \\
\hline P7 & $2 \%$ & $2 \%$ & $3 \%$ & $5 \%$ & $4 \%$ & $3 \%$ & $3 \%$ & $3 \%$ & $3 \%$ & $2 \%$ & $3 \%$ & $3 \%$ & $3 \%$ & $62 \%$ & $100 \%$ \\
\hline P8 & $2 \%$ & $2 \%$ & $3 \%$ & $6 \%$ & $3 \%$ & $3 \%$ & $3 \%$ & $3 \%$ & $3 \%$ & $2 \%$ & $3 \%$ & $3 \%$ & $3 \%$ & $61 \%$ & $100 \%$ \\
\hline P9 & $2 \%$ & $2 \%$ & $3 \%$ & $5 \%$ & $3 \%$ & $3 \%$ & $3 \%$ & $3 \%$ & $3 \%$ & $2 \%$ & $3 \%$ & $3 \%$ & $3 \%$ & $62 \%$ & $100 \%$ \\
\hline P10 & $3 \%$ & $2 \%$ & $3 \%$ & $6 \%$ & $3 \%$ & $3 \%$ & $3 \%$ & $3 \%$ & $3 \%$ & $3 \%$ & $3 \%$ & $4 \%$ & $3 \%$ & $61 \%$ & $100 \%$ \\
\hline P11 & $3 \%$ & $2 \%$ & $3 \%$ & $6 \%$ & $3 \%$ & $3 \%$ & $3 \%$ & $3 \%$ & $3 \%$ & $2 \%$ & $3 \%$ & $3 \%$ & $3 \%$ & $62 \%$ & $100 \%$ \\
\hline P12 & $2 \%$ & $2 \%$ & $3 \%$ & $6 \%$ & $3 \%$ & $3 \%$ & $3 \%$ & $3 \%$ & $3 \%$ & $2 \%$ & $3 \%$ & $3 \%$ & $3 \%$ & $62 \%$ & $100 \%$ \\
\hline P13 & $3 \%$ & $1 \%$ & $2 \%$ & $6 \%$ & $2 \%$ & $3 \%$ & $4 \%$ & $3 \%$ & $4 \%$ & $3 \%$ & $3 \%$ & $2 \%$ & $2 \%$ & $63 \%$ & $100 \%$ \\
\hline P14 & $3 \%$ & $2 \%$ & $3 \%$ & $6 \%$ & $3 \%$ & $3 \%$ & $3 \%$ & $3 \%$ & $3 \%$ & $2 \%$ & $3 \%$ & $3 \%$ & $3 \%$ & $61 \%$ & $100 \%$ \\
\hline P15 & $3 \%$ & $2 \%$ & $3 \%$ & $5 \%$ & $3 \%$ & $3 \%$ & $3 \%$ & $3 \%$ & $3 \%$ & $2 \%$ & $3 \%$ & $3 \%$ & $3 \%$ & $62 \%$ & $100 \%$ \\
\hline P16 & $3 \%$ & $2 \%$ & $3 \%$ & $5 \%$ & $3 \%$ & $3 \%$ & $3 \%$ & $3 \%$ & $3 \%$ & $2 \%$ & $3 \%$ & $3 \%$ & $3 \%$ & $61 \%$ & $100 \%$ \\
\hline P17 & $2 \%$ & $2 \%$ & $3 \%$ & $5 \%$ & $3 \%$ & $3 \%$ & $3 \%$ & $3 \%$ & $3 \%$ & $2 \%$ & $3 \%$ & $3 \%$ & $3 \%$ & $62 \%$ & $100 \%$ \\
\hline P18 & $3 \%$ & $1 \%$ & $2 \%$ & $5 \%$ & $2 \%$ & $3 \%$ & $3 \%$ & $3 \%$ & $4 \%$ & $3 \%$ & $3 \%$ & $3 \%$ & $3 \%$ & $64 \%$ & $100 \%$ \\
\hline P19 & $3 \%$ & $2 \%$ & $3 \%$ & $5 \%$ & $3 \%$ & $3 \%$ & $3 \%$ & $3 \%$ & $3 \%$ & $2 \%$ & $3 \%$ & $3 \%$ & $3 \%$ & $62 \%$ & $100 \%$ \\
\hline P20 & $3 \%$ & $2 \%$ & $3 \%$ & $5 \%$ & $3 \%$ & $3 \%$ & $3 \%$ & $3 \%$ & $3 \%$ & $2 \%$ & $3 \%$ & $4 \%$ & $3 \%$ & $62 \%$ & $100 \%$ \\
\hline P21 & $3 \%$ & $1 \%$ & $3 \%$ & $5 \%$ & $3 \%$ & $3 \%$ & $3 \%$ & $3 \%$ & $3 \%$ & $2 \%$ & $3 \%$ & $3 \%$ & $3 \%$ & $62 \%$ & $100 \%$ \\
\hline P22 & $2 \%$ & $1 \%$ & $3 \%$ & $5 \%$ & $3 \%$ & $3 \%$ & $3 \%$ & $3 \%$ & $3 \%$ & $3 \%$ & $3 \%$ & $3 \%$ & $3 \%$ & $63 \%$ & $100 \%$ \\
\hline $\mathbf{P 2 3}$ & $2 \%$ & $2 \%$ & $3 \%$ & $5 \%$ & $3 \%$ & $3 \%$ & $3 \%$ & $3 \%$ & $3 \%$ & $2 \%$ & $3 \%$ & $3 \%$ & $3 \%$ & $62 \%$ & $100 \%$ \\
\hline P24 & $3 \%$ & $2 \%$ & $3 \%$ & $5 \%$ & $3 \%$ & $3 \%$ & $3 \%$ & $3 \%$ & $3 \%$ & $3 \%$ & $3 \%$ & $3 \%$ & $3 \%$ & $61 \%$ & $100 \%$ \\
\hline P25 & $3 \%$ & $2 \%$ & $3 \%$ & $5 \%$ & $3 \%$ & $3 \%$ & $3 \%$ & $3 \%$ & $3 \%$ & $2 \%$ & $3 \%$ & $3 \%$ & $3 \%$ & $61 \%$ & $100 \%$ \\
\hline P26 & $3 \%$ & $2 \%$ & $3 \%$ & $6 \%$ & $3 \%$ & $3 \%$ & $3 \%$ & $3 \%$ & $3 \%$ & $3 \%$ & $3 \%$ & $3 \%$ & $3 \%$ & $62 \%$ & $100 \%$ \\
\hline P27 & $2 \%$ & $1 \%$ & $3 \%$ & $5 \%$ & $3 \%$ & $4 \%$ & $3 \%$ & $3 \%$ & $3 \%$ & $3 \%$ & $3 \%$ & $4 \%$ & $3 \%$ & $61 \%$ & $100 \%$ \\
\hline P28 & $3 \%$ & $1 \%$ & $3 \%$ & $5 \%$ & $2 \%$ & $3 \%$ & $3 \%$ & $3 \%$ & $3 \%$ & $2 \%$ & $3 \%$ & $4 \%$ & $3 \%$ & $63 \%$ & $100 \%$ \\
\hline P29 & $3 \%$ & $1 \%$ & $3 \%$ & $5 \%$ & $3 \%$ & $2 \%$ & $4 \%$ & $3 \%$ & $3 \%$ & $3 \%$ & $4 \%$ & $4 \%$ & $3 \%$ & $60 \%$ & $100 \%$ \\
\hline P30 & $2 \%$ & $1 \%$ & $4 \%$ & $4 \%$ & $4 \%$ & $3 \%$ & $3 \%$ & $3 \%$ & $3 \%$ & $3 \%$ & $3 \%$ & $4 \%$ & $3 \%$ & $61 \%$ & $100 \%$ \\
\hline P31 & $3 \%$ & $2 \%$ & $3 \%$ & $4 \%$ & $3 \%$ & $3 \%$ & $3 \%$ & $3 \%$ & $3 \%$ & $2 \%$ & $3 \%$ & $3 \%$ & $3 \%$ & $63 \%$ & $100 \%$ \\
\hline P32 & $3 \%$ & $2 \%$ & $3 \%$ & $4 \%$ & $3 \%$ & $3 \%$ & $3 \%$ & $3 \%$ & $3 \%$ & $3 \%$ & $3 \%$ & $3 \%$ & $3 \%$ & $62 \%$ & $100 \%$ \\
\hline P33 & $3 \%$ & $2 \%$ & $3 \%$ & $3 \%$ & $3 \%$ & $3 \%$ & $2 \%$ & $3 \%$ & $3 \%$ & $3 \%$ & $3 \%$ & $3 \%$ & $2 \%$ & $63 \%$ & $100 \%$ \\
\hline P34 & $3 \%$ & $2 \%$ & $3 \%$ & $4 \%$ & $3 \%$ & $3 \%$ & $3 \%$ & $3 \%$ & $2 \%$ & $3 \%$ & $3 \%$ & $3 \%$ & $3 \%$ & $62 \%$ & $100 \%$ \\
\hline P35 & $3 \%$ & $2 \%$ & $3 \%$ & $3 \%$ & $3 \%$ & $3 \%$ & $3 \%$ & $3 \%$ & $3 \%$ & $3 \%$ & $3 \%$ & $2 \%$ & $2 \%$ & $63 \%$ & $100 \%$ \\
\hline P36 & $2 \%$ & $1 \%$ & $3 \%$ & $4 \%$ & $4 \%$ & $3 \%$ & $3 \%$ & $2 \%$ & $3 \%$ & $3 \%$ & $3 \%$ & $4 \%$ & $3 \%$ & $62 \%$ & $100 \%$ \\
\hline P37 & $2 \%$ & $2 \%$ & $4 \%$ & $3 \%$ & $3 \%$ & $3 \%$ & $3 \%$ & $2 \%$ & $3 \%$ & $3 \%$ & $3 \%$ & $4 \%$ & $3 \%$ & $61 \%$ & $100 \%$ \\
\hline P38 & $4 \%$ & $1 \%$ & $4 \%$ & $4 \%$ & $3 \%$ & $3 \%$ & $3 \%$ & $3 \%$ & $3 \%$ & $3 \%$ & $3 \%$ & $4 \%$ & $3 \%$ & $60 \%$ & $100 \%$ \\
\hline P39 & $3 \%$ & $2 \%$ & $3 \%$ & $3 \%$ & $3 \%$ & $3 \%$ & $3 \%$ & $3 \%$ & $3 \%$ & $3 \%$ & $3 \%$ & $3 \%$ & $3 \%$ & $63 \%$ & $100 \%$ \\
\hline P40 & $3 \%$ & $2 \%$ & $3 \%$ & $3 \%$ & $3 \%$ & $3 \%$ & $3 \%$ & $3 \%$ & $3 \%$ & $3 \%$ & $3 \%$ & $3 \%$ & $3 \%$ & $63 \%$ & $100 \%$ \\
\hline P41 & $3 \%$ & $2 \%$ & $3 \%$ & $3 \%$ & $3 \%$ & $3 \%$ & $3 \%$ & $3 \%$ & $3 \%$ & $3 \%$ & $3 \%$ & $3 \%$ & $3 \%$ & $64 \%$ & $100 \%$ \\
\hline P42 & $3 \%$ & $2 \%$ & $3 \%$ & $3 \%$ & $3 \%$ & $3 \%$ & $3 \%$ & $3 \%$ & $3 \%$ & $3 \%$ & $3 \%$ & $3 \%$ & $3 \%$ & $63 \%$ & $100 \%$ \\
\hline P43 & $3 \%$ & $2 \%$ & $3 \%$ & $3 \%$ & $3 \%$ & $3 \%$ & $3 \%$ & $3 \%$ & $3 \%$ & $3 \%$ & $3 \%$ & $3 \%$ & $3 \%$ & $63 \%$ & $100 \%$ \\
\hline P44 & $3 \%$ & $2 \%$ & $3 \%$ & $3 \%$ & $3 \%$ & $3 \%$ & $3 \%$ & $3 \%$ & $3 \%$ & $3 \%$ & $3 \%$ & $3 \%$ & $2 \%$ & $64 \%$ & $100 \%$ \\
\hline P45 & $3 \%$ & $2 \%$ & $3 \%$ & $3 \%$ & $3 \%$ & $3 \%$ & $3 \%$ & $3 \%$ & $3 \%$ & $3 \%$ & $3 \%$ & $3 \%$ & $3 \%$ & $64 \%$ & $100 \%$ \\
\hline P46 & $3 \%$ & $2 \%$ & $3 \%$ & $3 \%$ & $3 \%$ & $3 \%$ & $3 \%$ & $3 \%$ & $3 \%$ & $3 \%$ & $3 \%$ & $3 \%$ & $3 \%$ & $63 \%$ & $100 \%$ \\
\hline P47 & $3 \%$ & $2 \%$ & $3 \%$ & $3 \%$ & $3 \%$ & $3 \%$ & $3 \%$ & $3 \%$ & $2 \%$ & $3 \%$ & $3 \%$ & $3 \%$ & $3 \%$ & $64 \%$ & $100 \%$ \\
\hline P48 & $3 \%$ & $2 \%$ & $3 \%$ & $3 \%$ & $3 \%$ & $3 \%$ & $3 \%$ & $3 \%$ & $3 \%$ & $3 \%$ & $3 \%$ & $3 \%$ & $3 \%$ & $64 \%$ & $100 \%$ \\
\hline P49 & $2 \%$ & $3 \%$ & $3 \%$ & $3 \%$ & $2 \%$ & $3 \%$ & $3 \%$ & $3 \%$ & $3 \%$ & $2 \%$ & $3 \%$ & $3 \%$ & $3 \%$ & $63 \%$ & $100 \%$ \\
\hline P50 & $2 \%$ & $3 \%$ & $3 \%$ & $3 \%$ & $3 \%$ & $3 \%$ & $3 \%$ & $2 \%$ & $3 \%$ & $2 \%$ & $3 \%$ & $3 \%$ & $5 \%$ & $62 \%$ & $100 \%$ \\
\hline
\end{tabular}


Table 18. The average weight of the Europe's portfolio diversified with assets of the African capital markets

\begin{tabular}{cccccc}
\hline & MV & RM & SV & MAD & FHS \\
\hline Namibia & $0 \%$ & $0 \%$ & $0 \%$ & $0 \%$ & $3 \%$ \\
Nigerian & $0 \%$ & $0 \%$ & $0 \%$ & $0 \%$ & $2 \%$ \\
Gongo & $12 \%$ & $10 \%$ & $24 \%$ & $43 \%$ & $3 \%$ \\
Cote D'Ivoire & $40 \%$ & $42 \%$ & $44 \%$ & $48 \%$ & $5 \%$ \\
Egypt & $0 \%$ & $0 \%$ & $1 \%$ & $1 \%$ & $3 \%$ \\
Morrocco & $0 \%$ & $0 \%$ & $0 \%$ & $0 \%$ & $3 \%$ \\
Tunisia & $8 \%$ & $5 \%$ & $9 \%$ & $1 \%$ & $3 \%$ \\
Botswana & $2 \%$ & $5 \%$ & $1 \%$ & $0 \%$ & $3 \%$ \\
Mauritius & $3 \%$ & $37 \%$ & $3 \%$ & $0 \%$ & $3 \%$ \\
Kenya & $0 \%$ & $0 \%$ & $0 \%$ & $0 \%$ & $3 \%$ \\
Uganda & $1 \%$ & $0 \%$ & $0 \%$ & $0 \%$ & $3 \%$ \\
Zambia & $24 \%$ & $0 \%$ & $14 \%$ & $6 \%$ & $3 \%$ \\
South Africa & $0 \%$ & $0 \%$ & $0 \%$ & $0 \%$ & $3 \%$ \\
UK & $0 \%$ & $0 \%$ & $0 \%$ & $0 \%$ & $3 \%$ \\
France & $0 \%$ & $0 \%$ & $0 \%$ & $0 \%$ & $2 \%$ \\
Italy & $0 \%$ & $0 \%$ & $0 \%$ & $0 \%$ & $2 \%$ \\
Spain & $0 \%$ & $0 \%$ & $0 \%$ & $0 \%$ & $2 \%$ \\
Austrian & $0 \%$ & $0 \%$ & $0 \%$ & $0 \%$ & $2 \%$ \\
SWISS & $0 \%$ & $0 \%$ & $0 \%$ & $0 \%$ & $3 \%$ \\
Belgium & $0 \%$ & $0 \%$ & $0 \%$ & $0 \%$ & $3 \%$ \\
Denmark & $2 \%$ & $0 \%$ & $0 \%$ & $0 \%$ & $3 \%$ \\
Finland & $0 \%$ & $0 \%$ & $0 \%$ & $0 \%$ & $3 \%$ \\
Ireland & $0 \%$ & $0 \%$ & $0 \%$ & $0 \%$ & $2 \%$ \\
Israel & $8 \%$ & $0 \%$ & $4 \%$ & $1 \%$ & $3 \%$ \\
Netherlands & $0 \%$ & $0 \%$ & $0 \%$ & $0 \%$ & $2 \%$ \\
Norway & $0 \%$ & $0 \%$ & $0 \%$ & $0 \%$ & $3 \%$ \\
Portugal & $0 \%$ & $0 \%$ & $0 \%$ & $0 \%$ & $2 \%$ \\
Sweden & $0 \%$ & $0 \%$ & $0 \%$ & $0 \%$ & $3 \%$ \\
Czech Republic & $0 \%$ & $0 \%$ & $0 \%$ & $0 \%$ & $3 \%$ \\
Greece & $0 \%$ & $0 \%$ & $0 \%$ & $0 \%$ & $2 \%$ \\
Hungary & $0 \%$ & $0 \%$ & $0 \%$ & $0 \%$ & $3 \%$ \\
Poland & $0 \%$ & $0 \%$ & $0 \%$ & $0 \%$ & $2 \%$ \\
Qatar & $0 \%$ & $0 \%$ & $0 \%$ & $0 \%$ & $3 \%$ \\
Russian & $0 \%$ & $0 \%$ & $0 \%$ & $0 \%$ & $3 \%$ \\
Turkey & $0 \%$ & $0 \%$ & $0 \%$ & $0 \%$ & $3 \%$ \\
UAE & $1 \%$ & $1 \%$ & $0 \%$ & $0 \%$ & $3 \%$ \\
Germany & $0 \%$ & $0 \%$ & $0 \%$ & $0 \%$ & $3 \%$ \\
\hline & & & & & \\
\hline & $0 \%$ & $0 \%$ & $0 \%$ & \\
& $0 \%$ & $0 \%$ & $0 \%$ & $0 \%$ \\
\hline
\end{tabular}

This table 18 shows us the average weights of European portfolio diversification with assets of African capital markets, according optimization models. To all optimization models, we can to see the high contribution of African markets in the portfolios, with featured for Gongo, Cote d'ivoire, Tunisia, Botswana, Mauritius and Zambia. We can to see either, better contribution of the Middlle East than Europen markets.

Table 19. The average Out-of-Sample performance

\begin{tabular}{|c|c|c|c|c|c|c|c|c|c|c|}
\hline \multirow[b]{2}{*}{ Models } & \multicolumn{5}{|c|}{ Europe } & \multicolumn{5}{|c|}{ Europe + Africa } \\
\hline & $\begin{array}{l}\text { Excess } \\
\text { Returns }\end{array}$ & Risk & $\begin{array}{c}\text { Downside } \\
\text { Risk }\end{array}$ & $\begin{array}{l}\text { Sharpe } \\
\text { Ratio }\end{array}$ & $\begin{array}{c}\text { Sortino } \\
\text { Ratio }\end{array}$ & $\begin{array}{l}\text { Excess } \\
\text { Returns }\end{array}$ & Risk & $\begin{array}{c}\text { Downside } \\
\text { Risk }\end{array}$ & $\begin{array}{c}\text { Sharpe } \\
\text { Ratio }\end{array}$ & $\begin{array}{c}\text { Sortino } \\
\text { Ratio }\end{array}$ \\
\hline MV & $0.005 \%$ & $2.245 \%$ & $1.498 \%$ & $0.282 \%$ & $0.469 \%$ & $0.071 \%$ & $1.116 \%$ & $0.574 \%$ & $6.203 \%$ & $12.548 \%$ \\
\hline $\mathbf{R M}$ & $0.102 \%$ & $2.039 \%$ & $1.141 \%$ & $5.428 \%$ & $9.341 \%$ & $0.213 \%$ & $1.062 \%$ & $0.567 \%$ & $20.582 \%$ & $38.118 \%$ \\
\hline SV & $0.010 \%$ & $1.498 \%$ & $1.498 \%$ & $1.080 \%$ & $1.080 \%$ & $0.113 \%$ & $0.574 \%$ & $0.574 \%$ & $19.875 \%$ & $19.875 \%$ \\
\hline MAD & $0.004 \%$ & $2.329 \%$ & $1.395 \%$ & $0.171 \%$ & $0.290 \%$ & $0.006 \%$ & $1.836 \%$ & $1.124 \%$ & $0.313 \%$ & $0.507 \%$ \\
\hline FHS & $0.003 \%$ & $5.162 \%$ & $4.659 \%$ & $0.067 \%$ & $0.069 \%$ & $0.012 \%$ & $4.060 \%$ & $3.794 \%$ & $0.320 \%$ & $0.317 \%$ \\
\hline
\end{tabular}

The table 19 shows the average Out-of-Sample performance of indicators Excess Return (ER). Risk (R). Sharpe Ratio (SR), and Sortino Ratio (S). The first sub-period came from August 05, 2004 to July 23, 2009 and the second sub-period came from July 30, 2009 to July 07, 2016. The risk-free rate corresponding to the mean return of the last market index of database. In this case correspond to the DAX index return of Germany. the 
Diversification of European investment portfolios with African assets shows better of the indicators than European investment portfolio. for all optimization models.

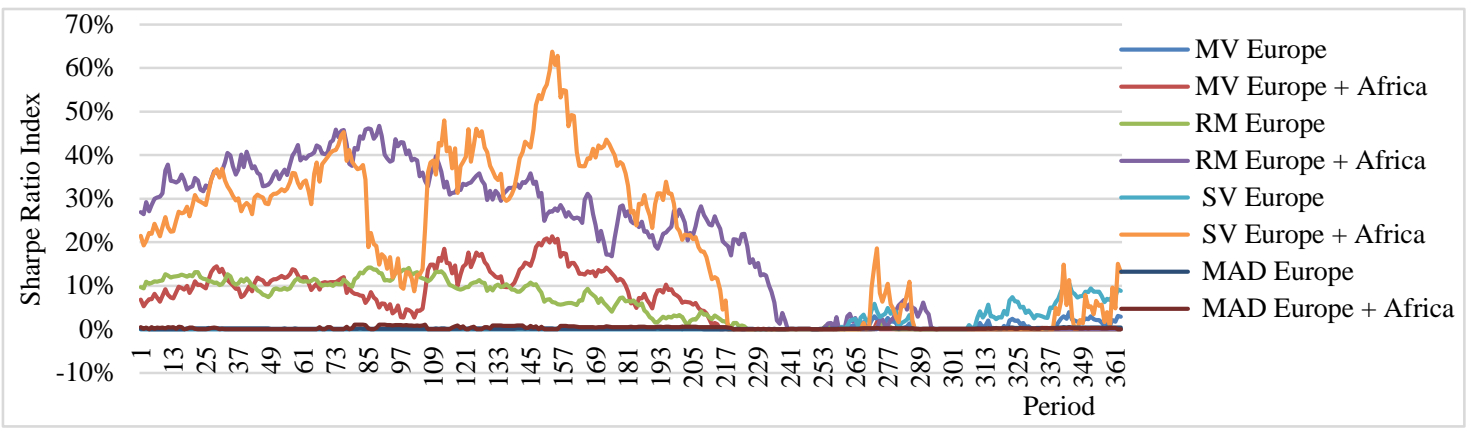

Figure 5. Out-of-Sample sharpe ratio performance

The figure 5 shows us the Out-of-Sample portfolio performances of the investment strategies for each optimization model measured by Sharpe Ratio a weekly. However, investment strategy with higher value of Sharpe Ratio, show better performance. For this analysis, we divide the database in two sub-period, being the first sub-period start from August 05, 2004 to July 23, /2009 and the second sub-period start from July 30, 2009 to July 07, /2016. In general the diversification strategy of European investment portfolio with African assets shown to have better performances than European investment portfolios, for all optimization models. However, if we look at the end of second period there are some European investment portfolios show better performance than diversification strategy of European investment portfolio with African assets.

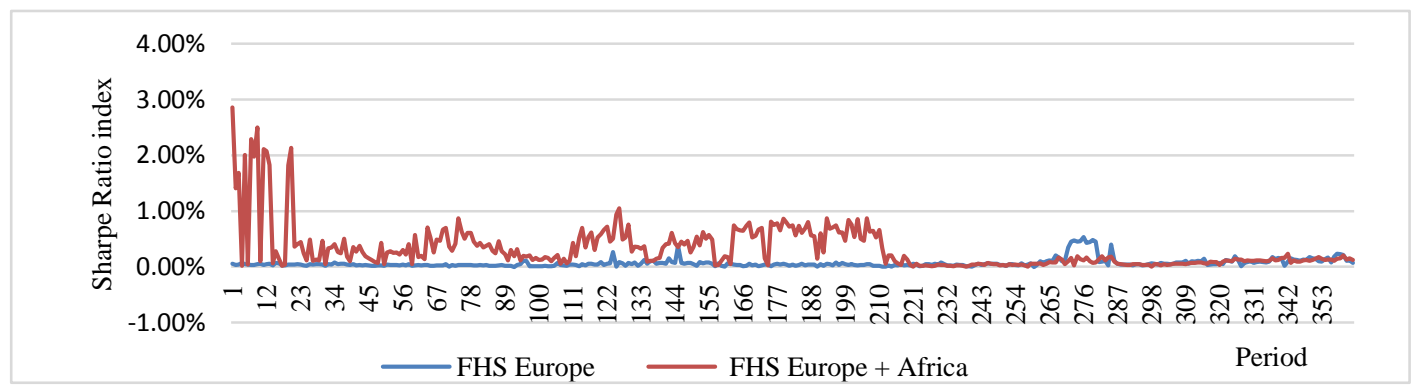

Figure 6. Out-of-Sample sharpe ratio performance by FHS method

The figure 6 shows us the Out-of-Sample portfolio performances of the investment strategies for each optimization model measured by Sharpe Ratio a weekly. We represent this method separated of others model because this is our contribution to the literature. His result fallow the tendencies of the others models. The diversification strategy of European investment portfolio with African assets shown to have more performances than European investment portfolios, for all optimization models. However, if we look at the end of second period there are some European investment portfolios show better performance than diversification strategy of European investment portfolio with African assets.

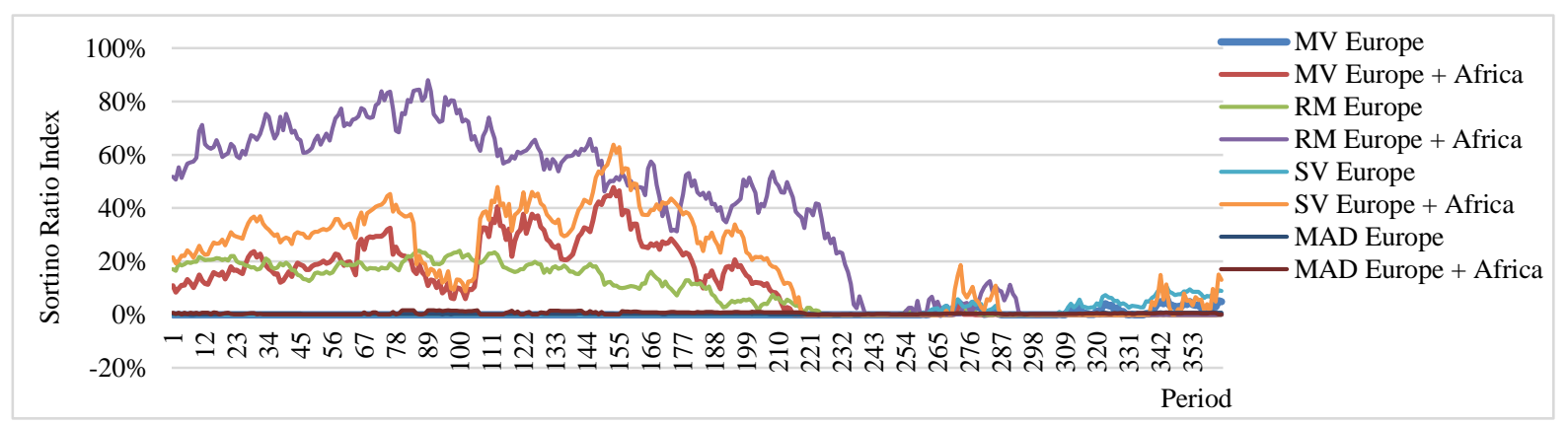

Figure 7. Out-of-Sample sortino ratio performance 
The figure 7 shows us the Out-of-Sample portfolio performances of the investment strategies for each optimization model measured by Sortino Ratio a weekly. However, investment strategy with higher value of Sharpe Ratio, show better performance. For this analysis, we divide the database in two sub-period, being the first sub-period start from August 05, 2004 to July 23, 2009 and the second sub-period start from July 30, 2009 to July 07,2016 . In general the diversification strategy of European investment portfolio with African assets shown to have better performances than European investment portfolios, for all optimization models. However, if we look at the end of second period there are some European investment portfolios show better performance than diversification strategy of European investment portfolio with African assets.

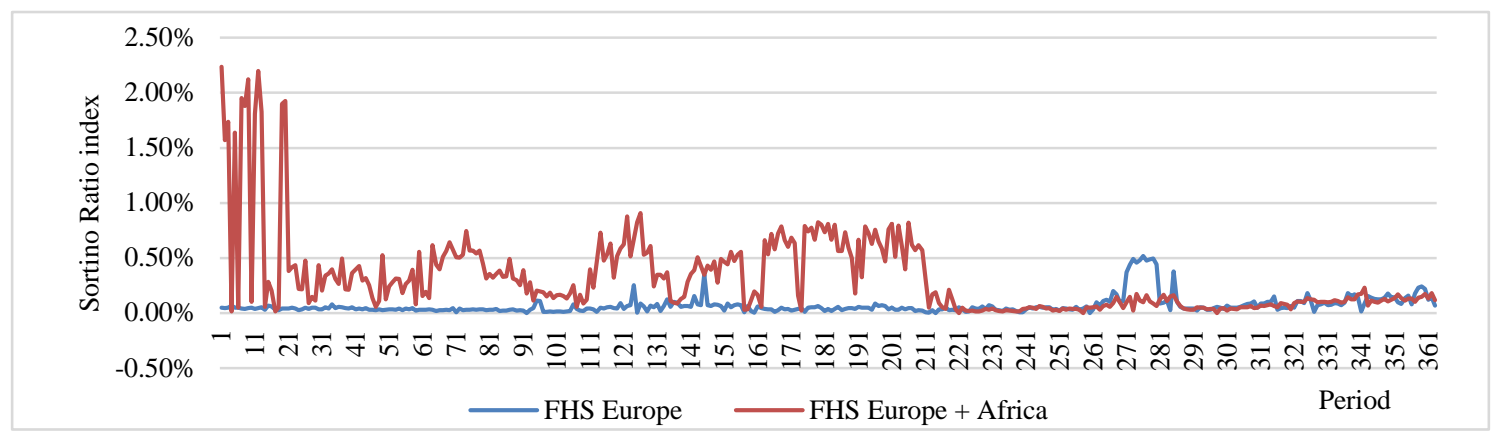

Figure 8. Out-of-Sample sortino ratio performance by FHS method

The figure 8 shows us the Out-of-Sample portfolio performances of the investment strategies for each optimization model measured by Sortino Ratio a weekly. We represent this method separated of others model because this is our contribution to the literature. His result fallow the tendencies of the others models. The diversification strategy of European investment portfolio with African assets shown to have more performances than European investment portfolios, for all optimization models. However, if we look at the end of second period there are some European investment portfolios show better performance than diversification strategy of European investment portfolio with African assets.

Table 20. Statistic test result Out-of-Sample performance

\begin{tabular}{ccccc}
\hline & \multicolumn{4}{c}{ Test result Sharpe Ratio performance } \\
\cline { 2 - 5 } Models & Null hypothesis & t-statistic & P-value & Reject or No reject \\
\hline Mean Variance (MV) & SR2>SR1 & 18.41 & 0.000 & No reject \\
Resample Michaud (RM) & SR2>SR1 & 17.13 & 0.000 & No reject \\
SemiVariance (SV) & SR2>SR1 & 20.45 & 0.000 & No reject \\
Mean Absolute Deviation (MAD) & SR2>SR1 & 8.82 & 0.000 & No reject \\
Filtered Historical Simulation (FHS) & SR2>SR1 & 11.66 & 0.000 & No reject \\
\hline & \multicolumn{4}{c}{ Test result Sortino Ratio performance } \\
\hline Models & Null hypothesis & t-statistic & P-value & Reject or No reject \\
\hline Mean Variance (MV) & S2>S1 & 17.90 & 0.0000 & No reject \\
Resample Michaud (RM) & S2>S1 & 17.72 & 0.0000 & No reject \\
SemiVariance (SV) & S2>S1 & 20.45 & 0.0000 & No reject \\
Mean Absolute Deviation (MAD) & S2>S1 & 8.54 & 0.0000 & No reject \\
Filtered Historical Simulation (FHS) & S2>S1 & 12.31 & 0.0000 & No reject
\end{tabular}

The table 20 shows us the statistical test result of the Out-of-Sample performance of the portfolio over of the 2 sub-period, being the first came from The first sub-period start from August 05, 2004 to July 23, 2009 and the second sub-period came from July 30, 2009 to July 07, 2016. The SR2 and S2 corresponding to the strategy 2 performances and SR1 and S1 and S1 are strategy 1 performances. Thus. was considered $1 \%$ significance level. We can to see high t-statistic value to all optimization models and p-value less $1 \%$, it that mean not rejection of null hypotheses. Then, we say that the Diversification of European investment portfolios with African assets presents better performance Out-Of -Sample than European investment portfolio without diversification.

On the other hand, these African companies can also use these financial resources to create new jobs that will encourage people stay in their country, which will also reduce illegal immigration. For example, like the tragedy of deaths in the seas of the Mediterranean as it has been happening where thousands of people lose their lives trying to cross seas in small boat in the hope to find better quality of live in Europe and help their family that are 
in Africa. Many of these people not reach the Europe due the bad travel conditions that ruin their life and dreams in the seas.

The result of this study can encourage the European investors to look at this problem and help Africa to solve it by buying African assets that can increase the value of their investment portfolios. The result of this study can to contribute in the same way to provide transfer of knowledge or idea to Africa through canalization of these investments; this is because sometimes where there are money transfer new idea are also shared. We can to say, that the result of this study can indirectly to contribute to eliminate the inequality between Europe and Africa through the European investors that looking to diversify their portfolios with African assets. On the other hand, this attitude on the part of European investors with the idea of buying African assets can to contribute in the poverty eradication in Africa.

Table 21. The average weight of the Europe's portfolio diversified with assets of African capital markets

\begin{tabular}{|c|c|c|c|c|c|}
\hline Markets & MV & $\mathbf{R M}$ & SV & MAD & FHS \\
\hline Namibia & $0 \%$ & $0 \%$ & $0 \%$ & $2 \%$ & $2 \%$ \\
\hline Nigerian & $1 \%$ & $1 \%$ & $0 \%$ & $1 \%$ & $1 \%$ \\
\hline Gongo & $39 \%$ & $25 \%$ & $58 \%$ & $4 \%$ & $7 \%$ \\
\hline Cote D'Tvoire & $2 \%$ & $1 \%$ & $3 \%$ & $6 \%$ & $8 \%$ \\
\hline Egypt & $0 \%$ & $0 \%$ & $0 \%$ & $2 \%$ & $2 \%$ \\
\hline Morrocco & $0 \%$ & $0 \%$ & $0 \%$ & $2 \%$ & $2 \%$ \\
\hline Tunisia & $15 \%$ & $25 \%$ & $13 \%$ & $3 \%$ & $3 \%$ \\
\hline Botswana & $9 \%$ & $14 \%$ & $4 \%$ & $2 \%$ & $3 \%$ \\
\hline Mauritius & $9 \%$ & $13 \%$ & $6 \%$ & $3 \%$ & $3 \%$ \\
\hline Kenya & $1 \%$ & $2 \%$ & $0 \%$ & $2 \%$ & $2 \%$ \\
\hline Uganda & $2 \%$ & $4 \%$ & $1 \%$ & $3 \%$ & $3 \%$ \\
\hline Zambia & $3 \%$ & $2 \%$ & $2 \%$ & $3 \%$ & $4 \%$ \\
\hline South Africa & $0 \%$ & $0 \%$ & $0 \%$ & $3 \%$ & $2 \%$ \\
\hline$U K$ & $0 \%$ & $0 \%$ & $0 \%$ & $2 \%$ & $2 \%$ \\
\hline France & $0 \%$ & $0 \%$ & $0 \%$ & $2 \%$ & $2 \%$ \\
\hline Italy & $0 \%$ & $0 \%$ & $0 \%$ & $2 \%$ & $1 \%$ \\
\hline Spain & $0 \%$ & $0 \%$ & $0 \%$ & $2 \%$ & $1 \%$ \\
\hline Austrian & $0 \%$ & $0 \%$ & $0 \%$ & $2 \%$ & $1 \%$ \\
\hline SWISS & $0 \%$ & $0 \%$ & $0 \%$ & $4 \%$ & $3 \%$ \\
\hline Belgium & $0 \%$ & $0 \%$ & $0 \%$ & $2 \%$ & $2 \%$ \\
\hline Denmark & $4 \%$ & $0 \%$ & $3 \%$ & $8 \%$ & $8 \%$ \\
\hline Finland & $0 \%$ & $0 \%$ & $0 \%$ & $2 \%$ & $2 \%$ \\
\hline Ireland & $1 \%$ & $0 \%$ & $0 \%$ & $4 \%$ & $4 \%$ \\
\hline Israel & $1 \%$ & $2 \%$ & $1 \%$ & $3 \%$ & $3 \%$ \\
\hline Netherlands & $0 \%$ & $0 \%$ & $0 \%$ & $2 \%$ & $2 \%$ \\
\hline Norway & $0 \%$ & $0 \%$ & $0 \%$ & $3 \%$ & $2 \%$ \\
\hline Portugal & $0 \%$ & $0 \%$ & $0 \%$ & $1 \%$ & $1 \%$ \\
\hline Sweden & $0 \%$ & $0 \%$ & $0 \%$ & $4 \%$ & $3 \%$ \\
\hline Czech Republic & $0 \%$ & $0 \%$ & $0 \%$ & $2 \%$ & $2 \%$ \\
\hline Greece & $1 \%$ & $0 \%$ & $0 \%$ & $1 \%$ & $1 \%$ \\
\hline Hungary & $0 \%$ & $0 \%$ & $0 \%$ & $2 \%$ & $1 \%$ \\
\hline Poland & $0 \%$ & $0 \%$ & $0 \%$ & $2 \%$ & $2 \%$ \\
\hline Qatar & $3 \%$ & $1 \%$ & $2 \%$ & $4 \%$ & $4 \%$ \\
\hline Russian & $0 \%$ & $0 \%$ & $0 \%$ & $2 \%$ & $2 \%$ \\
\hline Turkey & $0 \%$ & $0 \%$ & $0 \%$ & $3 \%$ & $2 \%$ \\
\hline$U A E$ & $7 \%$ & $9 \%$ & $4 \%$ & $4 \%$ & $5 \%$ \\
\hline Germany & $0 \%$ & $0 \%$ & $0 \%$ & $4 \%$ & $2 \%$ \\
\hline
\end{tabular}

This table 21 shows us the weekly average weights of European portfolio diversification with assets of African capital markets. according optimization models although Out-of-Sample analysis. To all optimization models. we can to see the high contribution of African markets in the portfolios, with featured for Gongo, Cote d'ivoire, Tunisia, Botswana, Mauritius, Uganda, and Zambia. We can to see either. better contribution of the Middlle East than Europen markets.

\section{References}

Abbes, M. B., \& Trichilli, Y. (2015). Islamic stock markets and potential diversification benefits. Borsa Istanbul Review, 15(2), 93-105. https://doi.org/10.1016/j.bir.2015.03.001 
Aldrich, J. (1997). R. A. Fisher and the Making of Maximum Likelihood 1912-1922. Statistical Science, 12(3), 162-176. https://doi.org/10.1214/ss/1030037906

Baele, L., \& Inghelbrecht, K. (2009). Time-varying integration and International diversification strategies. Journal of Empirical Finance, 16(3), 368-387. https://doi.org/10.1016/j.jempfin.2008.11.001

Bailey, B. A., Heck, J. L., \& Wilkens, K. A. (2005). International mutual fund performance and political risk. Review of Pacific Basin Financial Markets and Policies, 8(1), 167-184. https://doi.org/10.1142/S0219091505000312

Becker, F., Gürtler, M., \& Hibbeln, M. (2015). Markowitz versus Michaud: Portfolio optimization strategies reconsidered. The European Journal of Finance. https://doi.org/10.1080/1351847X.2013.830138

Bergin, P. R., \& Pyun, J. H. (2016). International portfolio diversification and multilateral effects of correlation. Journal of International Money and Finance, 62, 52-71. https://doi.org/10.1016/j.jimonfin.2015.12.012

Bessler, W., Opfer, H., \& Wolf, D. (2014). Multi-Asset Portfolio Optimization and Out-of-Sample Performance: An Evaluation of Black-Litterman, Mean Variance, and Naive Diversification Approaches. The European Journal of Finance, 23.

Bond, S. A., \& Satchell, S. E. (2002). Statistical properties of the sample Semi-variance. Applied Mathematical Finance, 9, 219-239. https://doi.org/10.1080/1350486022000015850

Bouslama, O., \& Ouda, O. B. (2014). International Portfolio Diversification Benefits: The Relevance of Emerging Markets. International Journal of Economics and Finance, 6(3). https://doi.org/10.5539/ijef.v6n3p200

Chiou, W. P. (2009). Benefits of international diversification with investment constraints: An over-time perspective. Journal of Multinational Financial Management, 19(2), 93-110. https://doi.org/10.1016/j.mulfin.2008.08.001

Coeurdacier, N., \& Guibaud, S. (2011). International portfolio diversification is better than you think. Journal of International Money and Finance, 30, 289-308. https://doi.org/10.1016/j.jimonfin.2010.10.003

Cumova, D., \& Nawrocki, D. (2011). A symmetric LPM model for heuristic mean-semivariance analysis. Journal of Economics and Business, 63, 217-236. https://doi.org/10.1016/j.jeconbus.2011.01.004

Dimitriou, D. I., \& Kenourgios, D. (2012). Opportunities for Diversification International in the Balkans Markets. International Journal of Economics and Research, 3(1).

Estrada, J. (2008). Mean-Semivariance optimization: A Heuristic Approach. Journal of Applied Finance (Formerly Financial Practice and Education), 18(1).

Fletcher, J. (2018). An empirical examination of the diversification benefits of U.K. international equity closed-end funds. International Review of Financial Analysis, 55, 23-34. https://doi.org/10.1016/j.irfa.2017.10.010

Giannopoulos, K., \& Tunaru, R. (2005). Coherent risk measures under filtered historical Simulation. Journal of Banking and Finance, 29, 979-996. https://doi.org/10.1016/j.jbankfin.2004.08.009

Hassan, K., Maroney, N. C., Monir, El-Sady, Hassan., \& Telfah, A. (2003). Country risk and stock market volatility, predictability, and diversification in the Middle East and Africa. Economic System, 27(1), 63-82. https://doi.org/10.1016/S0939-3625(03)00017-7

Jing-Hao, X., \& Titterington, M. (2011). The p-folded cumulative distribution function and the mean absolute deviation from the p-quantile. Statistics and Probability Letters, 81, 1179-1182. https://doi.org/10.1016/j.spl.2011.03.014

Konno, H., \& Yamazaki, H. (1991). Mean-Absolute Deviation Portfolio Optimization Model and ITS Applications to Tokyo Stock Market. Management Science, 37(5), 519-531. https://doi.org/10.1287/mnsc.37.5.519

Lagoarde-Segot, T., \& Lucey, B. M. (2007). International Portfolio Diversification: Is there a role for the Middle East and North Africa? Journal of Multinational Financial Management, (17), 401-416. https://doi.org/10.1016/j.mulfin.2007.01.001

Laopodis, N. T. (2005). Portfolio diversification benefits within Europe: Implications for a US investor. International Review of Financial Analysis, 14, 455-476. https://doi.org/10.1016/j.irfa.2004.10.012

Liang, Y., \& Mclntosh, W. (1999). Measuring the diversification benefit of an investment. Prudential Real Estate 
Investors.

Retrieved

from http://www2.prudential.com/o\&s/prei.nsf/14ef712a6b099d9d852566ef005111d0/344b7299e671a2be85257 08200739fe3/\$FILE/Measuring\%20Diversification.pdf

MacDowell, S. (2017). The benefits of international diversification: Re-examining the effect of market allocation constraints. The North American Journal of Economics and Finance, 41, 190-203. https://doi.org/10.1016/j.najef.2017.04.005

Majdoub, J., \& Mansour, W. (2013). Islamic equity market integration and volatility spillover between emerging and US stock markets. North American Journal of Economics and Finance, 29, 452-470. https://doi.org/10.1016/j.najef.2014.06.011

Mansourfar, G., Mohamad, S., \& Hassan, T. (2010). A review on international portfolio diversification: The Middle East and North Africa region. Africa Journal of Business Management, 4(19), 4167-4173.

Maria,,F. C., \& Eva, D. (2012). A fator analysis approach of international portfolio diversification: Does it pay off? Procedia Economics and Finance, 3, 648-653. https://doi.org/10.1016/S2212-5671(12)00209-2

Markowitz, H. M. (1952). Portfolio Selection. Journal of Finance, 7, 77-91.

Markowitz, H. M. (1959). Portfolio Selection. New York: John Wiley \& Sons.

Michaud, R. O. (1998). Efficient Asset Management: A Practical Guide to Stock Portfolio Optimization and Asset Allocation. Boston: Harvard Business School Press.

Moon, Y., \& Yao, T. (2011). A robust mean absolute deviation model for portfolio optimization. Computers \& Operations Research, 38, 1251-1258. https://doi.org/10.1016/j.cor.2010.10.020

Nie, U. S., Chong, C. W., Sambasivan, M., \& Nassir, A. M. (2014). The persistency of international diversification benefits: The role of the asymmetry volatility model. Asian Academy of Management Journal of Accounting \& Finance, 10(1), 151-165.

Rezayat, F., \& Yavas, B. F. (2006). International portfolio diversification: A study of linkages among the USA, European and Japanese equity market. Journal of Multinational Financial Management, 16(4), 440-458. https://doi.org/10.1016/j.mulfin.2005.10.002

Saiti, B., Bacha, O. I., \& Masih, M. (2014). The diversification benefits from Islamic investment during the financial turmoil: The case for the US-based equity investors. Borsa Istanbul Review, 4, $196-211$. https://doi.org/10.1016/j.bir.2014.08.002

Sharpe, W. F. (1994). The Sharpe Ratio. The Journal of Portfolio Management, 21(1), 49-58. https://doi.org/10.3905/jpm.1994.409501

\section{Notes}

Note 1. Apud Mansourfar et al. (2010).

Note 2. The study not included the African Islamic countries.

Note 3. The study included Egypt and Morocco.

Note 4. We consider this date to starte because as of this date, we find more number of possible markets to include in the study.

Note 5. This number of efficient portfolios is estimated through the optimization program for each optimization models.

Note 6. We propose as the benchmark risk-free rate asset, the average weekly interest rate of Treasury bills to monthly of USA bills during the data observation period.

Note 7. Risk of negative returns.

\section{Copyrights}

Copyright for this article is retained by the author(s), with first publication rights granted to the journal.

This is an open-access article distributed under the terms and conditions of the Creative Commons Attribution license (http://creativecommons.org/licenses/by/4.0/). 